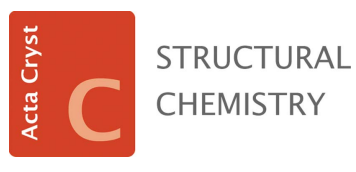

ISSN 2053-2296
Received 7 November 2016

Accepted 11 November 2016

Edited by A. L. Spek, Utrecht University, The Netherlands

Keywords: synthesis; kryptoracemate; fused-ring systems; dibenzoazepines; crystal structure; enantiomeric disorder; molecular structure; molecular conformation; hydrogen bonding.

CCDC references: $1516770 ; 1516769$; 1516768

Supporting information: this article has supporting information at journals.iucr.org/c

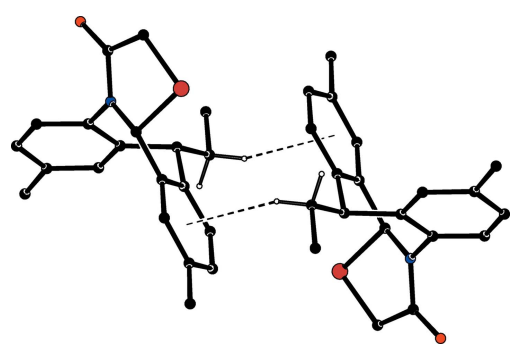

(C) 2017 International Union of Crystallography

\section{Three tetracyclic dibenzoazepine derivatives exhibiting different molecular conformations, different patterns of intermolecular hydrogen bonding and different modes of supramolecular aggregation}

\author{
Jeferson B. Mateus-Ruíz, ${ }^{a}$ Lina M. Acosta Quintero, ${ }^{a}$ Alirio Palma, ${ }^{a}$ Mario A. \\ Macías, ${ }^{\text {b Justo } \text { Cobo }^{c} \text { and Christopher Glidewell }}{ }^{\mathrm{d} *}$
}

\footnotetext{
aLaboratorio de Síntesis Orgánica, Escuela de Química, Universidad Industrial de Santander, AA 678 Bucaramanga, Colombia, 'b Departamento de Química, Universidad de los Andes, Carrera 1 No. 18A-12, Bogotá, Colombia, 'Departamento de Química Inorgánica y Orgánica, Universidad de Jaén, 23071 Jaén, Spain, and dSchool of Chemistry, University of St Andrews, Fife KY16 9ST, Scotland. *Correspondence e-mail: cg@st-andrews.ac.uk
}

The biological potential of compounds of the tricyclic dibenzo[ $[b, e]$ azepine system has resulted in considerable synthetic efforts to develop efficient methods for the synthesis of new derivatives of this kind. (9RS,15RS)-9-Ethyl11-methyl-9,13b-dihydrodibenzo[ $c$, $]$ thiazolo[3,2-a]azepin-3(2H)-one, $\mathrm{C}_{19} \mathrm{H}_{19} \mathrm{~N}$ OS, (I), crystallizes as a kryptoracemate with $Z^{\prime}=2$ in the space group $P 2_{1}$, with one molecule each of the $(9 R, 15 R)$ and $(9 S, 15 S)$ configurations in the asymmetric unit, while $(9 R S, 15 R S)$-9-ethyl-7,12-dimethyl-9,13b-dihydrodibenzo$[c, f]$ thiazolo[3,2-a] azepin-3(2H)-one, $\mathrm{C}_{20} \mathrm{H}_{21} \mathrm{NOS}$, (II), crystallizes with $Z^{\prime}=1$ in the space group $C 2 / c$. Ethyl (13RS)-2-chloro-13-ethyl-4-oxo-8,13-dihydro- $4 H$ benzo[5,6]azepino[3,2,1-ij]quinoline-5-carboxylate, $\mathrm{C}_{22} \mathrm{H}_{20} \mathrm{ClNO}_{3}$, (III), exhibits enantiomeric disorder in the space group $P \overline{1}$ such that the reference site is occupied by the $13 R$ and $13 S$ enantiomers, with occupancies of 0.900 (6) and 0.100 (6). In each of the two independent molecules in (I), the five-membered ring adopts an envelope conformation, but the corresponding ring in (II) adopts a half-chair conformation, while the six-membered ring in the major form of (III) adopts a twist-boat conformation. The conformation of the seven-membered ring in each of (I), (II) and the major form of (III) approximates to the twistboat form. The molecules of compound (I) are linked by two $\mathrm{C}-\mathrm{H} \cdots \mathrm{O}$ hydrogen bonds to form two independent antiparallel $C(5)$ chains, with each type containing only one enantiomer. These chains are linked into sheets by two $\mathrm{C}-\mathrm{H} \cdots \pi$ (arene) hydrogen bonds, in which the two donors are both provided by the $(9 R, 15 R)$ enantiomer and the two acceptor arene rings form part of a molecule of $(9 S, 15 S)$ configuration, precluding any additional crystallographic symmetry. The molecules of compound (II) are linked by inversion-related C$\mathrm{H} \cdots \pi$ (arene) hydrogen bonds to form isolated cyclic centrosymmetric dimers. The molecules of compound (III) are linked into cyclic centrosymmetric dimers by $\mathrm{C}-\mathrm{H} \cdots \mathrm{O}$ hydrogen bonds and these dimers are linked into chains by a $\pi-\pi$ stacking interaction. Comparisons are made with some related structures.

\section{Introduction}

The tricyclic dibenzo[ $b, e]$ azepine system constitutes a class of nitrogen-containing heterocyclic compounds whose chemistry continues to be of interest, because of the action of compounds containing this system as analgesics and as anticancer, antidepressive, antihistaminic, antimuscarinic and antipsychotic agents (Al-Qawasmeh et al., 2009). Examples of such compounds in current clinical use include mianserin, racemic 2-methyl-1,2,3,4,10,14b-hexahydrodibenzo[c,f]pyrazino[1,2-a]- 
azepine, which is a potent antidepressant (Dinesh et al., 2014), and epinastine, racemic 3-amino-9,13b-dihydro- $1 H$-dibenz$[c, f]$ imidazo[1,5- $a]$ azepine, which is an antihistaminic used in the treatment of allergic conjunctivitis (Liu et al., 2004). The biological potential of these compounds has resulted in considerable synthetic efforts to develop efficient methods for the synthesis of new derivatives of this kind (Andrés et al., 2002; Stappers et al., 2002; Wikström et al., 2002).

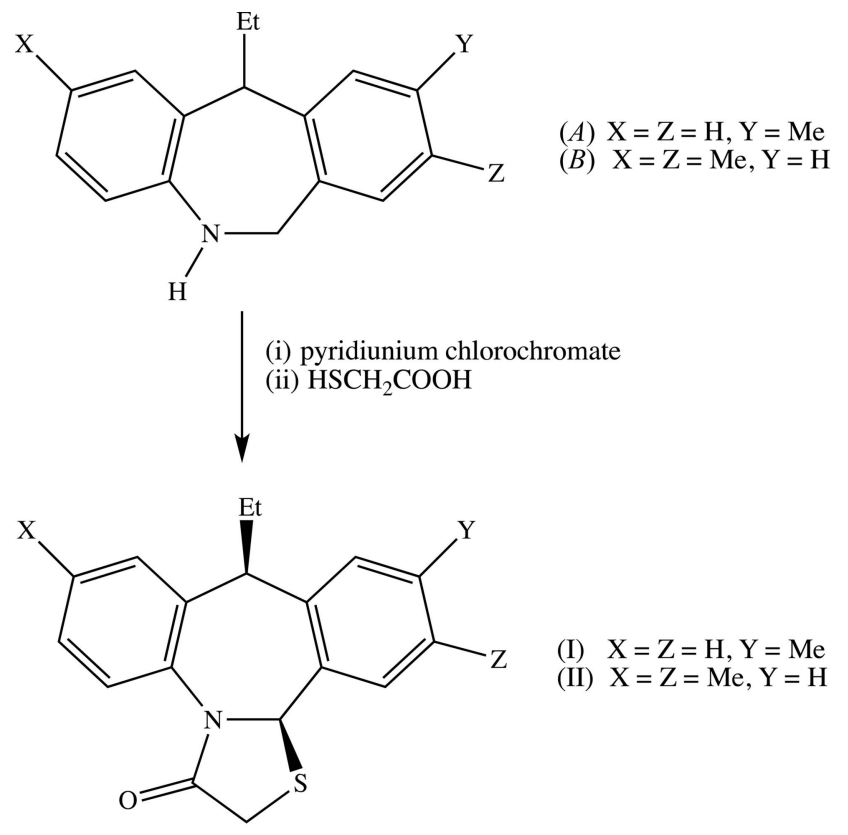

Scheme 1

In this context, and as part of our own interest in the identification of other molecular entities with pharmacological potential, we have for several years studied the chemistry of the synthetically available dihydrodibenzo[ $b, e]$ azepines (Palma et al., 2004) as building blocks for the construction of novel fused tetracyclic azepine systems. Accordingly, we have reported the synthesis of tetrahydrodibenzo $[c, f]$ thiazolo[3,2-a]azepine derivatives, compounds in which the dibenzo[ $[b, e]$ azepine nucleus is fused to a thiazolidin-4-one ring (Palma et al., 2010). We are now developing a simple and efficient synthetic methodology for the preparation of derivatives of the type alkyl 4-oxobenzo[5,6]azepino[3,2,1-ij]quinoline-5-carboxylate. This is a new heterocyclic system in which a benzazepine nucleus is fused to a 4-quinolone system, which is also of great interest for both the medicinal chemistry and pharmaceutical industries (Mugnaini et al., 2009), mainly because of their antibacterial activity, the best studied biological property of the so-called fluoroquinolone antibiotics.

We report here the molecular and supramolecular structures of three compounds containing fused tetracyclic azepine systems, namely 9 -ethyl-11-methyl-9,13b-dihydrodibenzo $[c, f]$ thiazolo[3,2-a]azepin-3(2H)-one, (I), 9-ethyl-7,12-dimethyl9,13b-dihydrodibenzo[ $[c, f]$ thiazolo[3,2-a]azepin-3(2H)-one, (II), and ethyl 2-chloro-13-ethyl-4-oxo-8,13-dihydro-4H-benzo[5,6]azepino[3,2,1-ij]quinoline-5-carboxylate, (III) (Figs. 1-3). Compounds (I) and (II) were synthesized from the corresponding dihydrodibenzo[b,e]azepines $(A)$ and $(B)$ (see
Scheme 1) according to a previously described procedure (Palma et al., 2010), in which the tricyclic precursors $(A)$ and $(B)$ were first subjected to oxidation using pyridinium chlorochromate, followed by cyclocondensation with thioglycolic acid to give (I) and (II). Compound (III) was synthesized from dihydrodibenzo[b,e]azepine $(C)$ employing the modified Gould-Jacobs reaction, in which an alkoxymethylenemalonate derivative, here diethyl 2-(methoxymethylene)malonate, reacts with the amino group of the precursor with displacement of the ethoxy unit by the $\mathrm{N}$ atom giving the intermediate $(D)$, followed by benzannulation to give the quinolone derivative (III) (see Scheme 2).<smiles>CCC1c2ccccc2CNc2ccc(Cl)cc21</smiles>

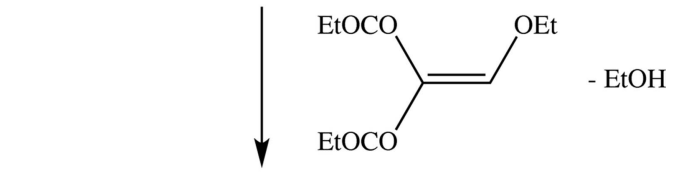

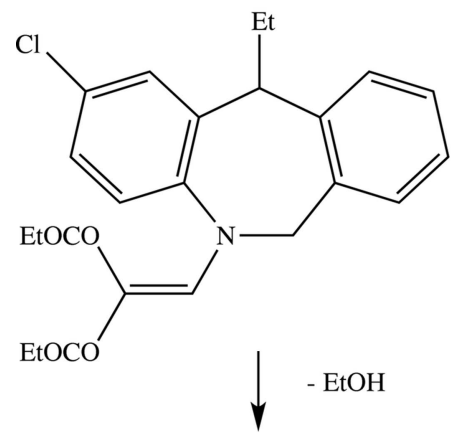

(D)

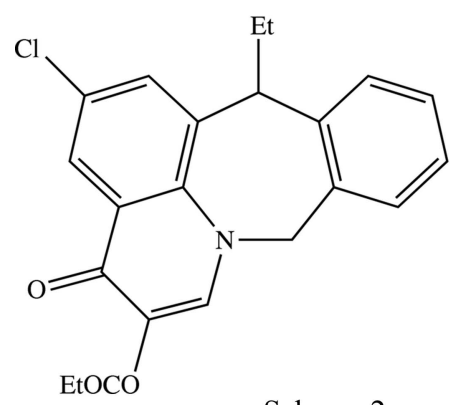

\section{Experimental}

\subsection{Synthesis and crystallization}

Compounds (I) and (II) were prepared according to the method reported previously by Palma et al. (2010). For the synthesis of compound (III), a solution of 2-chloro-11-ethyl6,11-dihydro- $5 H$-dibenzo[ $b, e]$ azepine, $(C)(0.10 \mathrm{mmol})$, and diethyl 2-(methoxymethylene)malonate $(0.13 \mathrm{mmol})$ in toluene $(10 \mathrm{ml})$ was heated under reflux for $15 \mathrm{~h}$ until the reaction was complete, as indicated by thin-layer chromatography 
Table 1

Experimental details.

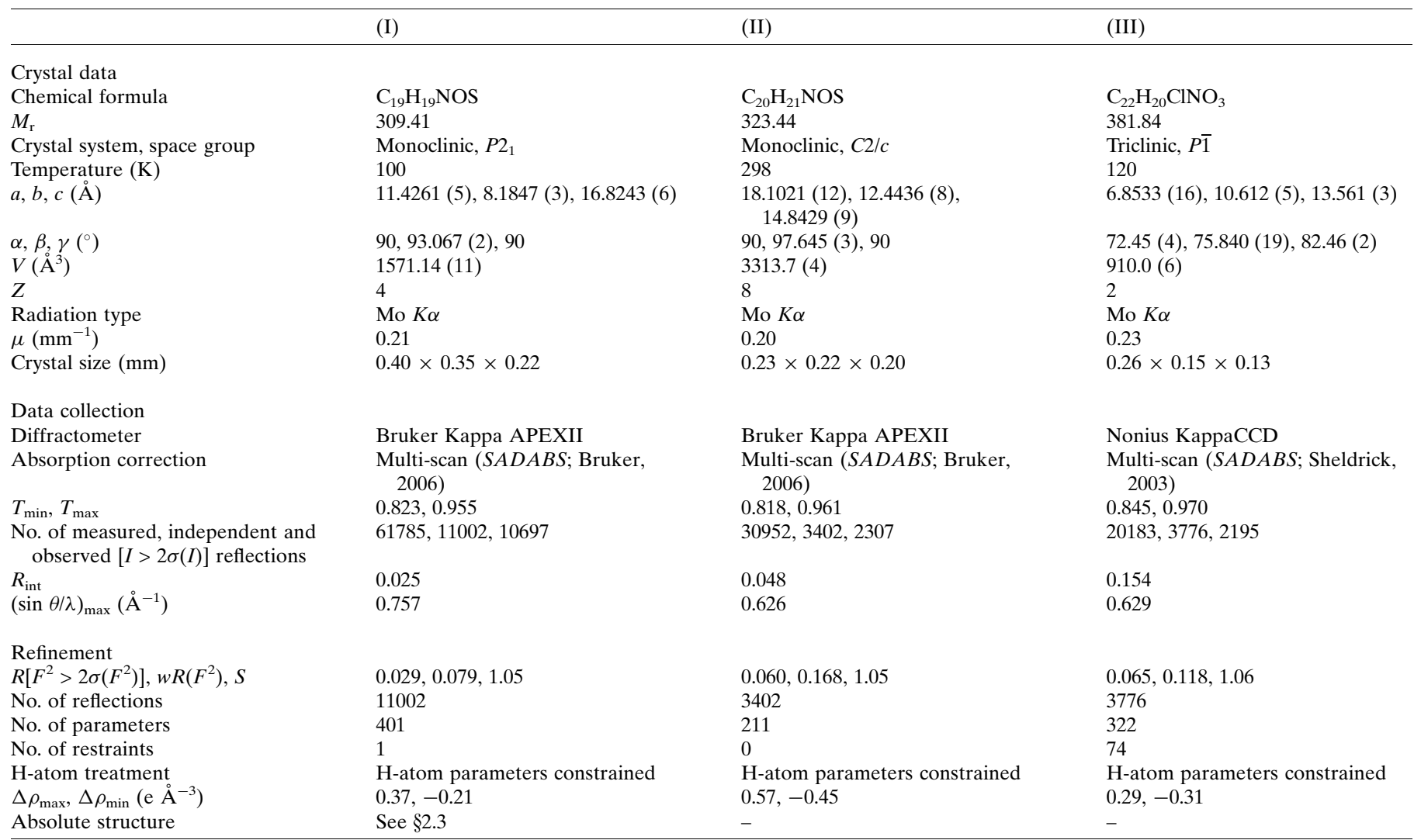

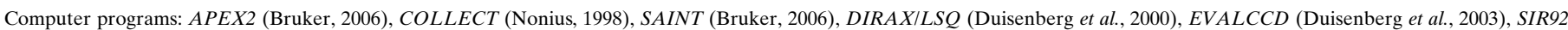
(Altomare et al., 1994), SIR2014 (Burla et al., 2015), SHELXL2014 (Sheldrick, 2015) and PLATON (Spek, 2009).

(TLC). The solvent and the excess of diethyl 2-(methoxymethylene)malonate were removed from the reaction mixture under reduced pressure, and Eaton's reagent, i.e. a $7.7 \%$ solution of phosphorus $(\mathrm{V})$ oxide in methanesulfonic acid $(1.6 \mathrm{ml})$, was added to the remaining crude material. This mixture was heated at $343 \mathrm{~K}$ for $40 \mathrm{~min}$, again with TLC monitoring, then cooled to ambient temperature and neutralized with saturated aqueous sodium carbonate solution. The neutralized mixture was extracted with ethyl acetate $(3 \times 50 \mathrm{ml})$ and the combined organic extracts were dried over anhydrous sodium sulfate. The solvent was removed under reduced pressure and the crude product was purified by column chromatography on silica gel using heptane-ethyl acetate mixtures $(10: 1$ to $1: 3 \mathrm{v} / \mathrm{v})$ to give compound (III) (yield 80\%, m.p. 466-467 K). Colourless crystals of compounds (I)-(III) suitable for single-crystal $\mathrm{X}$-ray diffraction were grown by slow evaporation, at ambient temperature and in the presence of air, of solutions in heptane-ethyl acetate $(2: 1 \mathrm{v} / \mathrm{v})$ for (I) and (II), and in ethanolethyl acetate $(7: 3 v / v)$ for (III).

\subsection{Spectroscopic data}

$R_{\mathrm{F}}=0.27$ (ethyl acetate-heptane, $\left.1: 1 \mathrm{v} / \mathrm{v}\right) ; \mathrm{IR}\left(\mathrm{cm}^{-1}\right): 2965-$ $2926(\mathrm{C}-\mathrm{H}), 1687$ [C=O(ester)], $1605[\mathrm{C}=\mathrm{O}$ (ketone) $], 1482$ $(\mathrm{C}=\mathrm{C}), 1146(\mathrm{C}-\mathrm{O})$; $\mathrm{NMR}\left(\mathrm{CDCl}_{3}\right): \delta\left({ }^{1} \mathrm{H}\right) 1.02(t, J=7.2 \mathrm{~Hz}$, $\left.3 \mathrm{H}, 13-\mathrm{CH}_{2}-\mathrm{CH}_{3}\right), 1.41\left(t, J=7.2 \mathrm{~Hz}, 3 \mathrm{H}, \mathrm{O}-\mathrm{CH}_{2}-\mathrm{CH}_{3}\right)$, 2.36-2.24 ( $\left.m, 2 \mathrm{H}, 13-\mathrm{CH}_{2}-\mathrm{CH}_{3}\right), 4.10$ (br s, 1H, 13-H), 4.39 $\left(q, J=7.2 \mathrm{~Hz}, 2 \mathrm{H}, \mathrm{O}-\mathrm{CH}_{2}-\right), 4.88\left(\right.$ br s $\left., 1 \mathrm{H}, 8-\mathrm{H}_{\mathrm{B}}\right), 5.88($ br s , $\left.1 \mathrm{H}, 8-\mathrm{H}_{\mathrm{A}}\right), 7.22(d d, J=7.4,1.4 \mathrm{~Hz}, 1 \mathrm{H}, 12-\mathrm{H}), 7.31(t d, J=7.4$, $1.4 \mathrm{~Hz}, 1 \mathrm{H}, 10-\mathrm{H}), 7.36(t d, J=7.4,1.4 \mathrm{~Hz}, 1 \mathrm{H}, 11-\mathrm{H}), 7.40$ (dd, $J=7.4,1.4 \mathrm{~Hz}, 1 \mathrm{H}, 9-\mathrm{H}), 7.51(d, J=2.4 \mathrm{~Hz}, 1 \mathrm{H}, 1-\mathrm{H}), 8.34(d$, $J=2.4 \mathrm{~Hz}, 1 \mathrm{H}, 3-\mathrm{H}), 8.53(s, 1 \mathrm{H}, 6-\mathrm{H}) ; \delta\left({ }^{13} \mathrm{C}\right) 13.1\left(13-\mathrm{CH}_{2}-\right.$ $\left.\mathrm{CH}_{3}\right), 14.5\left(\mathrm{O}-\mathrm{CH}_{2}-\mathrm{CH}_{3}\right), 61.1\left(\mathrm{O}-\mathrm{CH}_{2}-\right), 61.2(8-\mathrm{C})$, 109.6 (5-C), 126.3 (3-C), 128.1 (10-C), 129.1 (9-C, 12-C), 129.8 (11-C), 131.2 (2-C), 131.8 (8a-C), 132.3 (3a-C), 134.2 (13a-C), 137.2 (3b-C), 140.5 (12a-C), 150.1 (6-C), 165.6 (COO), 172.8 (4-C); HRMS (EI-MS, $70 \mathrm{eV}$ ) $\mathrm{m} / \mathrm{z}$ found 381.1132, $\mathrm{C}_{22} \mathrm{H}_{20}{ }^{35} \mathrm{ClNO}_{3}$ requires 381.1132 .

\subsection{Refinement}

Crystal data, data collection and structure refinement details are summarized in Table 1. For compounds (I) and (II), all $\mathrm{H}$ atoms were located in difference maps and subsequently treated as riding atoms in geometrically idealized positions, with $\mathrm{C}-\mathrm{H}$ distances of 0.95 (aromatic), 0.98 (methyl), 0.99 (methylene) or $1.00 \AA$ (methine) for (I), and 0.93, 0.96, 0.97 or $0.98 \AA$ for the corresponding bond types in (II), and with, in each case, $U_{\text {iso }}(\mathrm{H})=k U_{\text {eq }}(\mathrm{C})$, where $k=1.5$ for the methyl groups, which were permitted to rotate but not to tilt, and 1.2 for all other $\mathrm{H}$ atoms. The correct absolute configuration for 
compound (I) was established using both the Flack $x$ parameter (Flack, 1983), $x=0.007$ (6), calculated (Parsons et al., 2013) using 4787 quotients of the type $\left[\left(I^{+}\right)-\left(I^{-}\right)\right] /\left[\left(I^{+}\right)+\left(I^{-}\right)\right]$, and the Hooft $y$ parameter (Hooft et al., 2010) $y=0.001$ (7). It was apparent from an early stage in the refinement of compound (III) that the molecules exhibited configurational disorder, such that the reference site was occupied by partialoccupancy molecules of both $R$ and $S$ configuration having markedly unequal occupancies. For the minor component, having the $S$ configuration, the bonded distances and the 1,3 nonbonded distances were restrained to be the same as the corresponding distances in the major component, having an $R$ configuration, subject to s.u. values of 0.01 and $0.02 \AA$, respectively; in addition, the anisotropic displacement parameters for pairs of atoms occupying similar regions of physical space were constrained to be identical. The $\mathrm{H}$ atoms in the major component were all located in difference maps and then treated as riding atoms in geometrically idealized positions, with $\mathrm{C}-\mathrm{H}=0.95$ (alkenyl and aromatic), 0.98 (methyl), 0.99 (methylene) or $1.00 \AA$ (methine), and with $U_{\text {iso }}(\mathrm{H})$ defined as for (I) and (II). In the final analysis of variance for compound (II), there was a negative value, -0.346 , of $K=\operatorname{mean}\left(F_{\mathrm{o}}{ }^{2}\right) /$ mean $\left(F_{\mathrm{c}}^{2}\right)$ for the group of 409 very weak reflections having $F_{\mathrm{c}} / F_{\mathrm{c}}(\max )$ in the range $0.000<F_{\mathrm{c}} / F_{\mathrm{c}}(\max )<0.006$, and for compound (III) there was a large value, 5.504, of $K$ for the group of 382 very weak reflections having $F_{\mathrm{c}} / F_{\mathrm{c}}(\max )$ in the range $0.000<F_{\mathrm{c}} / F_{\mathrm{c}}(\max )<0.015$.

\section{Results and discussion}

The constitutions of compounds (I) and (II) are rather similar, differing only in the number and location of the methyl substituents, which are at position 11 in (I) and at positions 7 and 12 in (II). Despite this close similarity, compound (I) crystallizes with $Z^{\prime}=2$ in the Sohncke space group $P 2_{1}$, while (II) crystallizes in the centrosymmetric space group $C 2 / c$. In molecule 1 of compound (I), containing atom S11 (Fig. 1a), there are stereogenic centres at atoms $\mathrm{C} 19$ and $\mathrm{C} 115$; the reference molecule 1 was selected as one having the $R$ configuration at atom $\mathrm{C} 19$ and on this basis the configuration at atom $\mathrm{C} 115$ is also $R$, whereas the configurations at atoms $\mathrm{C} 29$ and C215 in molecule 2 (Fig. $1 b$ ) are both $S$. Thus, despite crystallizing in the space group $P 2_{1}$, compound (I) is a racemic mixture of $(9 R, 15 R)$ and $(9 S, 15 S)$ enantiomers and it is therefore a kryptoracemate (Morales \& Fronczek, 1996; Fábián \& Brock, 2010; Bernal \& Watkins, 2015); a search for possible additional crystallographic symmetry found none. Compound (III) has a stereogenic centre at position 13 and the reference molecule was selected as one having the $R$ configuration at this site. However, it was apparent that the reference site was in fact occupied by partial-occupancy molecules of both $R$ and $S$ configurations (Figs. $3 a$ and $3 b$ ), having occupancies of $0.900(6)$ and 0.100 (6), respectively. These two enantiomeric forms occupy similar but not quite identical locations (Fig. 3c). The centrosymmetric space groups of compounds (II) and (III) confirm that these compounds have both crystallized as racemic mixtures. That

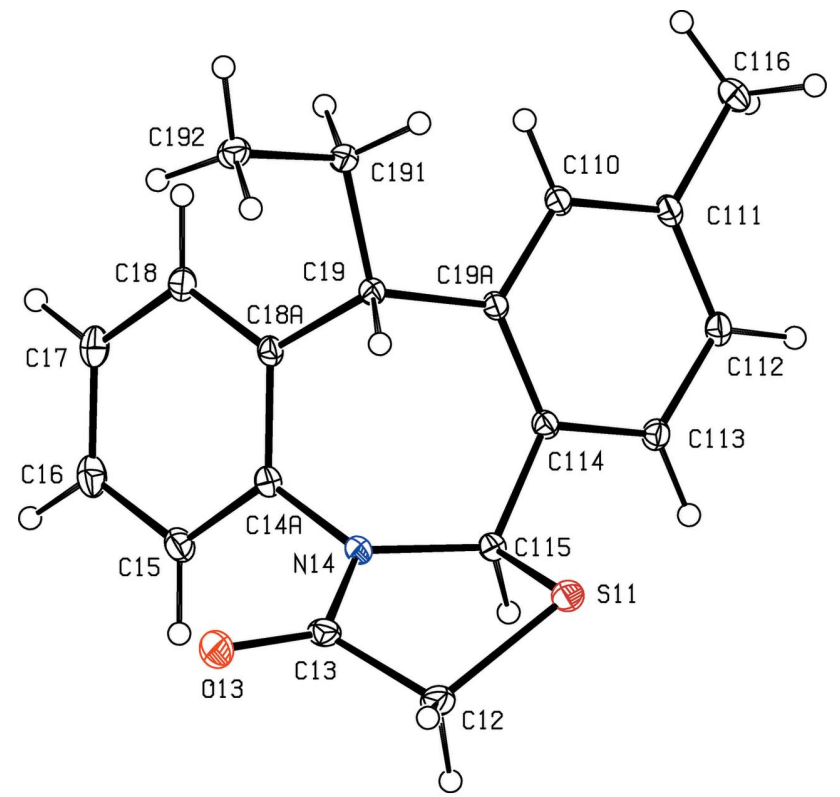

(a)

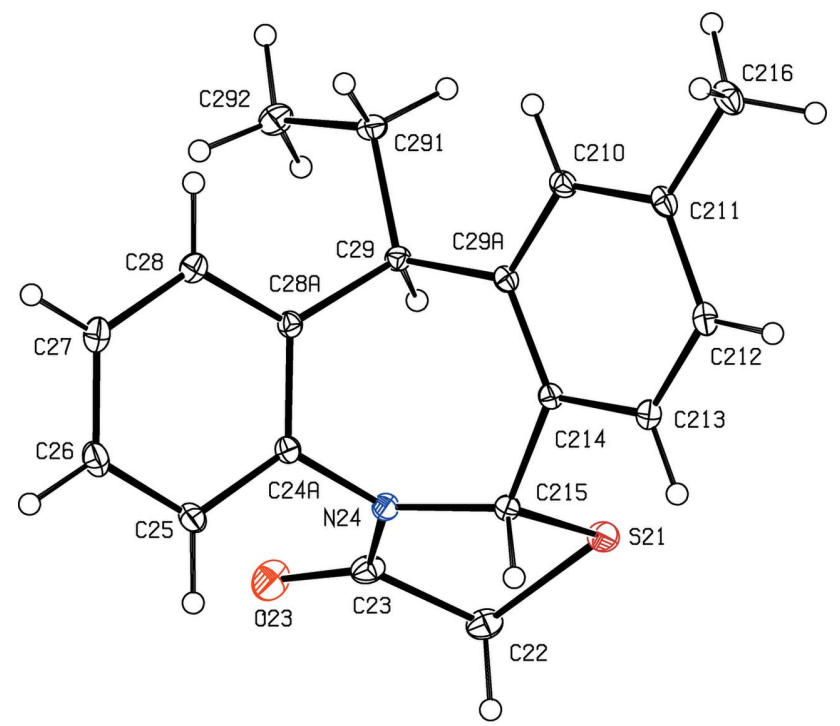

Figure 1

(b)

The molecular structures of the two independent molecules of compound (I), showing ( $a$ ) molecule 1 , which has the $(9 R, 15 R)$ configuration, and $(b)$ molecule 2 , which has the $(9 S, 15 S)$ configuration. Displacement ellipsoids are drawn at the $30 \%$ probability level.

compounds (I)-(III) are racemic is expected from the racemic nature of the precursors $(A)-(C)$ (Palma et al., 2010), but it is interesting to note that for compounds (I) and (II), the stereochemistry at position 15 appears to be wholly controlled by that at position 9 and no evidence was found for the formation of the diastereoisomeric $(9 R S, 15 S R)$ forms.

In each of the two independent molecules of compound (I), the five-membered ring is slightly puckered out of planarity, and the ring-puckering parameters (Cremer \& Pople, 1975) show that in each molecule this ring adopts an envelope (Evans \& Boeyens, 1989) conformation (Table 2), with the ring folded across the line $\mathrm{C} x 2-\mathrm{C} x 15$, where $x=1$ or 2 in molecules 1 and 2, respectively; the difference of $c a 180^{\circ}$ 
Table 2

Selected geometric parameters $\left(\AA{ }^{\circ}{ }^{\circ}\right)$.

(I), molecule 1

$0.4627(12)$

359.12 (17)

(I), molecule 2

(II)

(III), major

(III), minor

Ring-puckering parameters

Five-membered ring

$Q_{2}$

$\varphi_{2}$

Six-membered ring

$Q$

$\theta$

$\varphi$

Seven-membered rings

$Q$
$\varphi_{2}$

$\varphi_{3}$

Dihedral angles

$70.84(4)$

$-169.01(11)$

$0.3979(12)$

178.1 (2)
$0.300(3)$

$339.0(5)$

Torsion angles

$\mathrm{C} x 9 A-\mathrm{C} x 9-\mathrm{C} x 91-\mathrm{C} x 92$

Cy $2 A-\mathrm{C} y 13-\mathrm{C} y 31-\mathrm{C} y 32$

$\begin{array}{lll}0.9540(13) & 0.9418(12) & 0.981(3) \\ 38.32(8) & 217.47(8) & 40.16(16) \\ 286.0(3) & 107.8(3) & 281.6(7) \\ 70.84(4) & 65.44(4) & 76.67(9) \\ -169.01(11) & & \\ & 170.60(10) & -173.0(2)\end{array}$

$0.107(8)$
$87(4)$

39 (4)

$16(7)$

$112(26)$

$2(30)$

$0.85(5)$

267 (4)

17 (7)

$66(3)$

$\begin{array}{ll}0.843(6) & 0.85(5) \\ 271.8(4) & 267(4) \\ 9.6(10) & 17(7) \\ 52.8(2) & 66(3)\end{array}$

$-69.4(5)$

$42(5)$

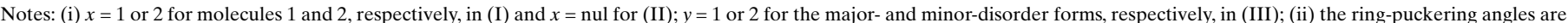

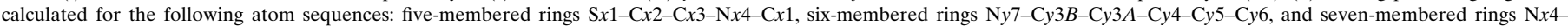

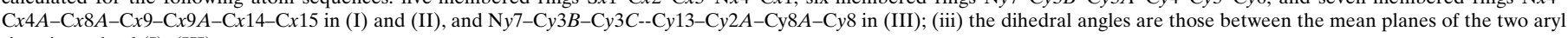
rings in each of (I)-(III)

between the $\varphi_{2}$ values for the two molecules in (I) confirms their enantiomeric relationship. By contrast with (I), the fivemembered ring in compound (II) adopts a half-chair conformation in which the ring is twisted about a line through atom $\mathrm{C} 3$ and the approximate mid-point of the $\mathrm{S} 1-\mathrm{C} 15$ bond. For idealized half-chair and envelope conformations, the values of $\varphi_{2}$ are $(36 k+18)^{\circ}$ and $36 k^{\circ}$, respectively, where $k$ represents an integer. Within the major disorder form of compound (III), the six-membered heterocyclic ring is slightly puckered into a twist-boat conformation; for an idealized twist-boat conformation; the ring-puckering angles are $\theta=90^{\circ}$ and $\varphi=(60 k+$ $30)^{\circ}$, where $k$ represents an integer. For the seven-membered ring in each of (I), (II) and the major form of (III), the ring conformations are dominated by the twist-boat $\sin$ form 2

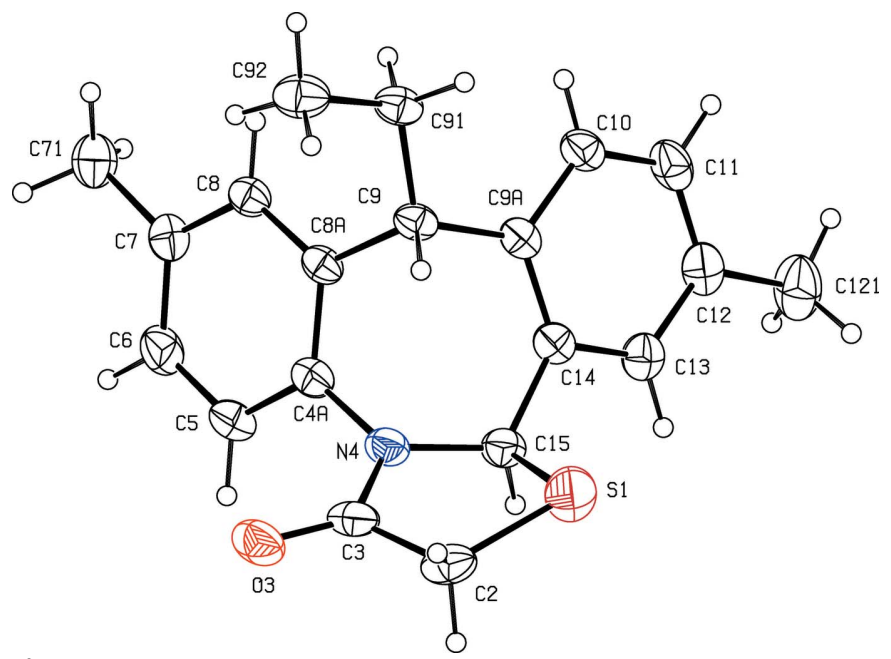

Figure 2

The molecular structure of the $(9 R, 15 R)$ enantiomer of compound (II). Displacement ellipsoids are drawn at the $30 \%$ probability level.
(Evans \& Boeyens, 1989). By contrast, the most common conformation of the seven-membered ring in tricyclic dibenzazepines is one intermediate between the boat and twistboat forms (Sanabría et al., 2014), while the most common form in benzopyrimidoazepines is the boat form ( $\cos$ form 2) (Acosta et al., 2015; Acosta Quintero, Palma et al., 2016).

In each of the five independent molecular entities reported here, the dihedral angle between the two aryl rings falls within a fairly narrow range of less than $25^{\circ}$ (Table 2 ). The specification of the molecular conformations is completed by the orientation of the ethyl substituent relative to the sevenmembered ring; the torsion angles defining this orientation are very similar in compounds (I) and (II), except for the difference in sign between the two independent molecules in (I) consistent with their enantiomeric relationship, whereas in (III) this orientation is entirely different (Table 2 and Figs. 1-3).

Table 3

Parameters $\left(\AA{ }^{\circ},{ }^{\circ}\right)$ for hydrogen bonds and short intermolecular contacts.

$C g 1-C g 3$ represent the centroids of the $\mathrm{C} 29 A / \mathrm{C} 210-\mathrm{C} 214, \mathrm{C} 24 A / \mathrm{C} 25-\mathrm{C} 28 /$ $\mathrm{C} 28 A$ and $\mathrm{C} 9 A / \mathrm{C} 10-\mathrm{C} 14$ rings, respectively.

\begin{tabular}{llllll}
\hline Compound & $D-\mathrm{H} \cdots A$ & $D-\mathrm{H}$ & $\mathrm{H} \cdots A$ & $D \cdots A$ & $D-\mathrm{H} \cdots A$ \\
\hline (I) & $\mathrm{C} 115-\mathrm{H} 115 \cdots \mathrm{O} 13^{\mathrm{i}}$ & 1.00 & 2.47 & $3.3084(18)$ & 141 \\
& $\mathrm{C} 215-\mathrm{H} 215 \cdots \mathrm{O} 23^{\text {ii }}$ & 1.00 & 2.37 & $3.1681(18)$ & 136 \\
& $\mathrm{C} 112-\mathrm{H} 112 \cdots C g 1^{i i i}$ & 0.95 & 2.81 & $3.6738(13)$ & 152 \\
& $\mathrm{C} 113-\mathrm{H} 113 \cdots C g 2^{\mathrm{iii}}$ & 0.95 & 2.85 & $3.7676(14)$ & 163 \\
(II) & $\mathrm{C} 2-\mathrm{H} 2 B \cdots \mathrm{O} 3^{\text {iv }}$ & 0.97 & 2.57 & $3.120(4)$ & 116 \\
& $\mathrm{C} 91-\mathrm{H} 91 A \cdots C g 3^{\mathrm{v}}$ & 0.97 & 2.82 & $3.736(3)$ & 157 \\
(III) & $\mathrm{C} 18-\mathrm{H} 18 B \cdots \mathrm{O} 151^{\text {vi }}$ & 0.99 & 2.54 & $3.483(9)$ & 159 \\
& $\mathrm{C} 19-\mathrm{H} 19 \cdots \mathrm{O} 151^{\text {vi }}$ & 0.95 & 2.54 & $3.396(12)$ & 149 \\
& $\mathrm{C} 28-\mathrm{H} 28 B \cdots \mathrm{O} 251^{\text {vi }}$ & 0.99 & 2.10 & $3.13(7)$ & 159 \\
& $\mathrm{C} 29-\mathrm{H} 29 \cdots \mathrm{O} 251^{\text {vi }}$ & 0.95 & 2.75 & $3.34(12)$ & 124
\end{tabular}

Symmetry codes: (i) $-x, y+\frac{1}{2},-z+1$; (ii) $-x, y-\frac{1}{2},-z$; (iii) $x, y+1, z$; (iv) $-x+\frac{1}{2},-y+\frac{1}{2}$, $-z+1 ;$ (v) $-x+1,-y+1,-z+1$; (vi) $-x+1,-y+2,-z+1$. 


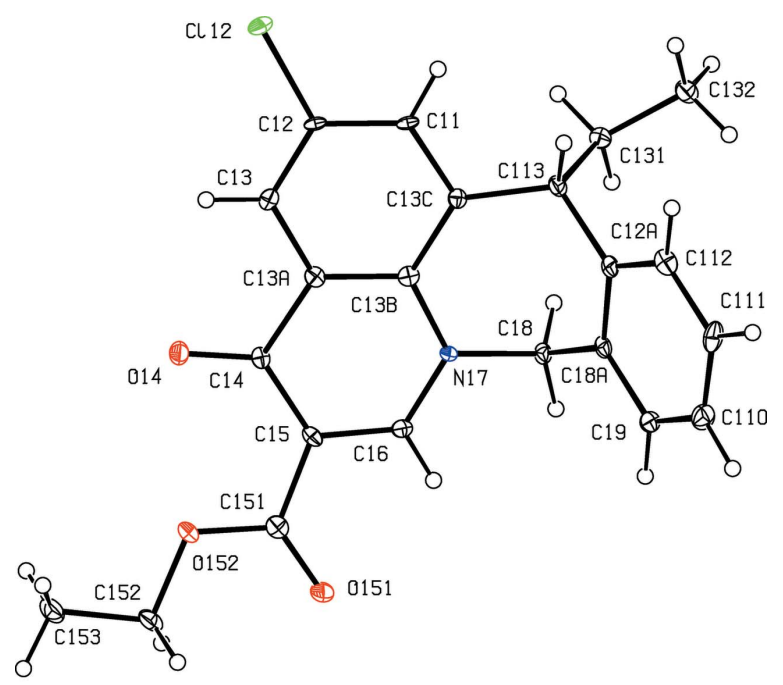

(a)

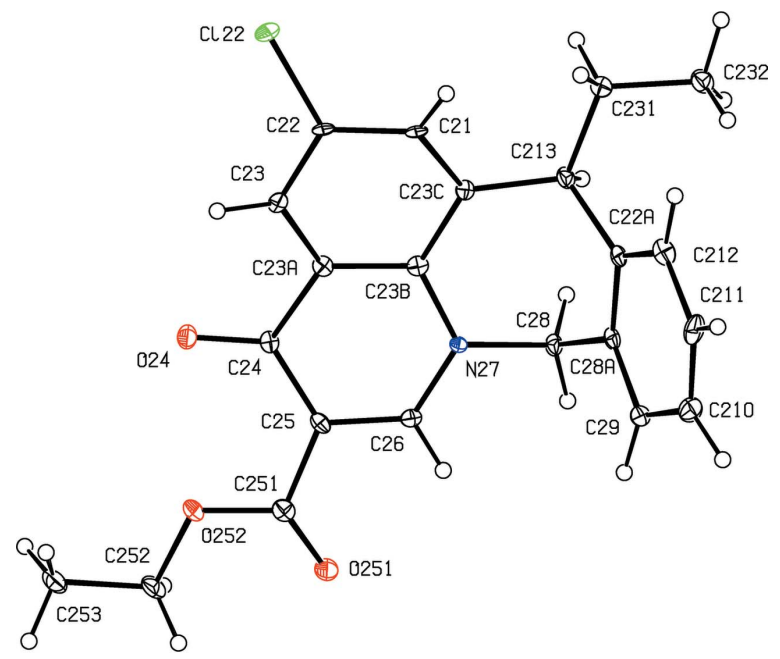

(b)

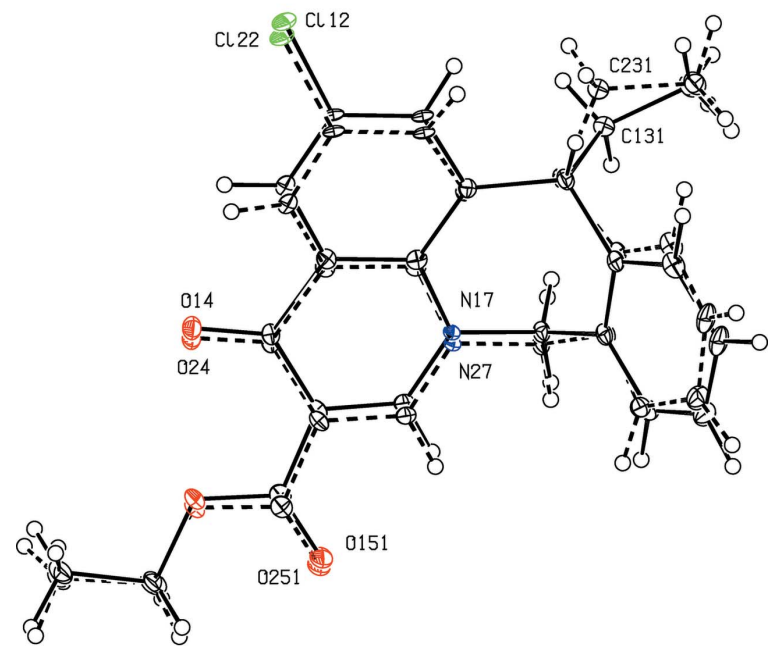

Figure 3

(c)

The molecular structures of the disordered components of compound (III), showing ( $a$ ) the major $R$ enantiomer, $(b)$ the minor $S$ enantiomer and $(c)$ the two disorder components together, with the bonds in the major form shown as full lines and those in the minor form shown as broken lines. Displacement ellipsoids are drawn at the $30 \%$ probability level and, for the sake of clarity, the majority of the atom labels have been omitted from part $(c)$.
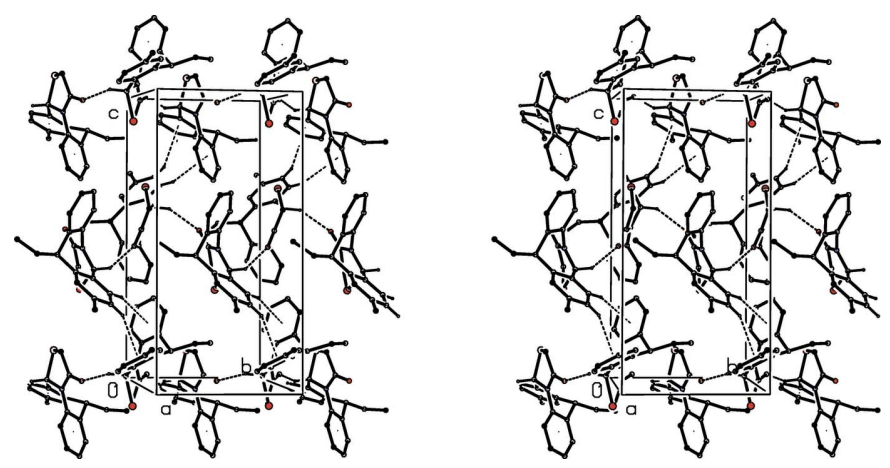

Figure 4

A stereoview of part of the crystal structure of compound (I), showing the formation of a hydrogen-bonded sheet parallel to (100) in which chains built from $\mathrm{C}-\mathrm{H} \cdots \mathrm{O}$ hydrogen bonds are linked by $\mathrm{C}-\mathrm{H} \cdots \pi$ (arene) hydrogen bonds. For the sake of clarity, $\mathrm{H}$ atoms which are not involved in the motifs shown have been omitted.

Despite the close similarity between the constitutions of compounds (I) and (II), the supramolecular assembly in these two compounds is entirely different. In compound (I), the molecules are linked into complex sheets by a combination of two $\mathrm{C}-\mathrm{H} \cdots \mathrm{O}$ hydrogen bonds and two $\mathrm{C}-\mathrm{H} \cdots \pi($ arene) hydrogen bonds (Table 3), but the formation of the sheet structure is readily analysed in terms of simple substructures (Ferguson et al., 1998a,b; Gregson et al., 2000). The molecules of type 1 which are related by the $2_{1}$ screw axis along $\left(0, y, \frac{1}{2}\right)$ are linked by $\mathrm{C}-\mathrm{H} \cdots \mathrm{O}$ hydrogen bonds to form a $C(5)$

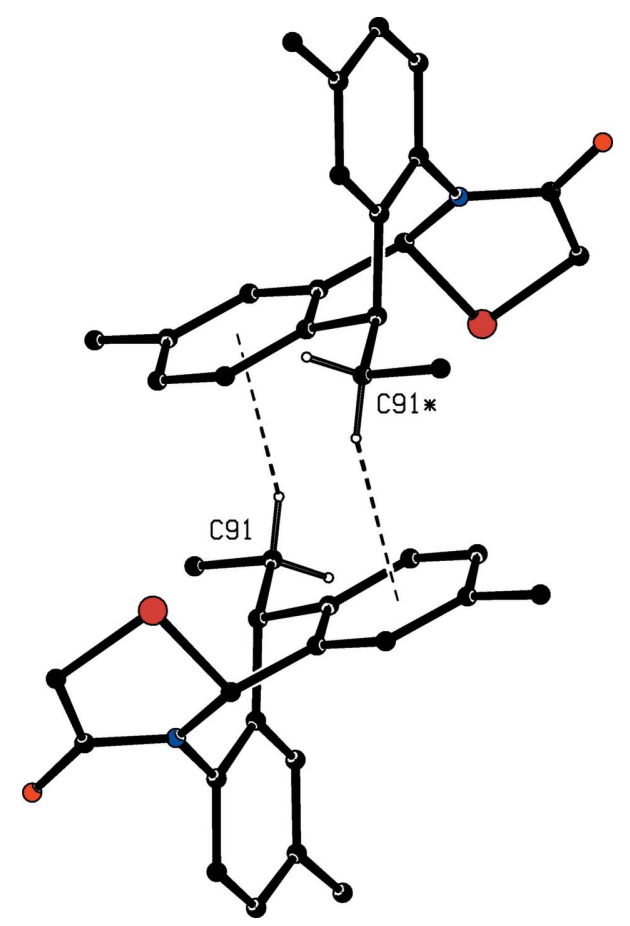

Figure 5

Part of the crystal structure of compound (II), showing the formation of a centrosymmetric hydrogen-bonded dimer. For the sake of clarity, the unit-cell outline and $\mathrm{H}$ atoms bonded to $\mathrm{C}$ atoms that are not involved in the motif shown have been omitted. The atom marked with an asterisk $(*)$ is at the symmetry position $(-x+1,-y+1,-z+1)$. 


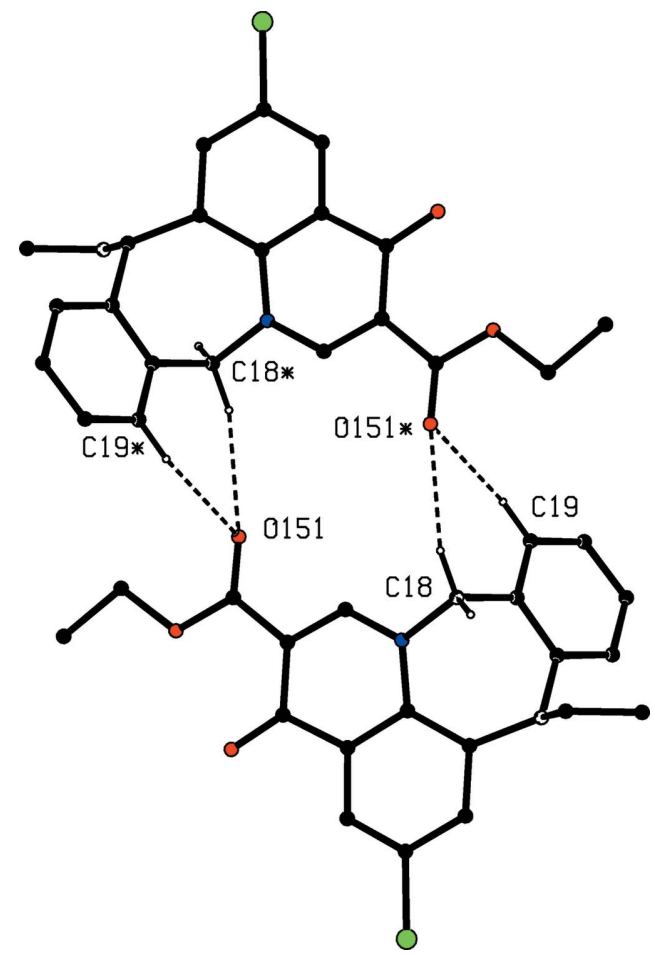

Figure 6

Part of the crystal structure of compound (III), showing the formation by the major-disorder form of a cyclic centrosymmetric hydrogen-bonded dimer. For the sake of clarity, the unit-cell outline and $\mathrm{H}$ atoms bonded to $\mathrm{C}$ atoms that are not involved in the motif shown have been omitted. Atoms marked with an asterisk $(*)$ are at the symmetry position $(-x+1$, $-y+2,-z+1)$.

(Bernstein et al., 1995) chain running parallel to the [010] direction. A similar $C(5)$ chain, antiparallel to the first chain is built from type 2 molecules which are related by the $2_{1}$ screw axis along $(0, y, 0)$; thus each type of $C(5)$ chain contains only a single enantiomeric form. Two independent $\mathrm{C}-\mathrm{H} \cdots \pi$ (arene) hydrogen bonds, in which the donors are two adjacent $\mathrm{C}-\mathrm{H}$ bonds in a type 1 molecule and the acceptors are the two aryl rings of a type 2 molecule, thus precluding the possibility of any additional crystallographic symmetry, link the type 1 chains along $\left(0, y, n+\frac{1}{2}\right)$ to the type 2 chains along $(0, y, n)$, where $n$ represents an integer in each case, to form a sheet lying parallel to (100) (Fig. 4); however, there are no directionspecific interactions between adjacent sheets.

In contrast to the complex supramolecular assembly in compound (I), that in compound (II) is extremely simple. Inversion-related pairs of molecules are linked by paired $\mathrm{C}-$ $\mathrm{H} \cdot \cdots \pi$ (arene) hydrogen bonds to form centrosymmetric dimers, each containing an enantiomeric pair (Fig. 5), but there are no direction-specific interactions between adjacent dimers, so that the supramolecular assembly is finite and zerodimensional.

The molecules of compound (III) are linked by $\mathrm{C}-\mathrm{H} \cdots \mathrm{O}$ hydrogen bonds to form centrosymmetric dimers (Fig. 6). For the major-disorder form, the hydrogen bonds generate a dimer, centred at $\left(\frac{1}{2} 1, \frac{1}{2}\right)$, characterized by an outer $R_{2}^{2}(18)$ ring, which encloses an inner $R_{2}^{2}(14)$ ring flanked by two inversion- related $R_{2}^{1}(6)$ rings; for the minor-disorder component, only the $R_{2}^{2}(14)$ ring is present as the $\mathrm{H} 29 \cdots \mathrm{O} 251^{\mathrm{i}}$ separation [symmetry code: (i) $-x+1,-y+2,-z+1$ ] of $2.75 \AA$ is above the sum of the van der Waals radii (Rowland \& Taylor, 1996), so that the corresponding $\mathrm{C}-\mathrm{H} \cdots \mathrm{O}$ contact cannot be regarded as a hydrogen bond. Dimers of this type are linked into a chain by a single $\pi-\pi$ stacking interaction. The chlorinated aryl rings of the molecules at $(x, y, z)$ and $(-x+2,-y+1$, $-z+1)$ are strictly parallel, with an interplanar spacing of 3.368 (3) $\AA$; the ring-centroid separation is 3.649 (4) $\AA$, corresponding to a ring-centroid offset of 1.404 (4) $\AA$. This interaction links hydrogen-bonded dimers related by translation into a $\pi$-stacked chain running parallel to the [1] 0 ] direction (Fig. 7).<smiles>C[C@@H]1Cc2c(Cl)ncnc2N2CCc3cccc1c32</smiles>

(IV)

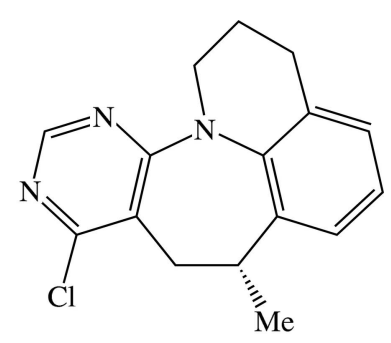

(V)
Scheme 3

It is interesting briefly to compare compounds (I)-(III) reported here with the related tetracyclic benzopyrimidoazepine derivatives (IV) and (V) (see Scheme 3). Firstly, the syntheses of (IV) and (V) utilized a completely different approach (Acosta Quintero et al., 2015; Acosta Quintero, Burgos et al., 2016) from that employed for the preparation of (I)-(III); the synthesis of compounds (I)-(III) appended an additional ring to a preformed dibenzazepine skeleton, while those for (IV) and (V) were based on the formation of the
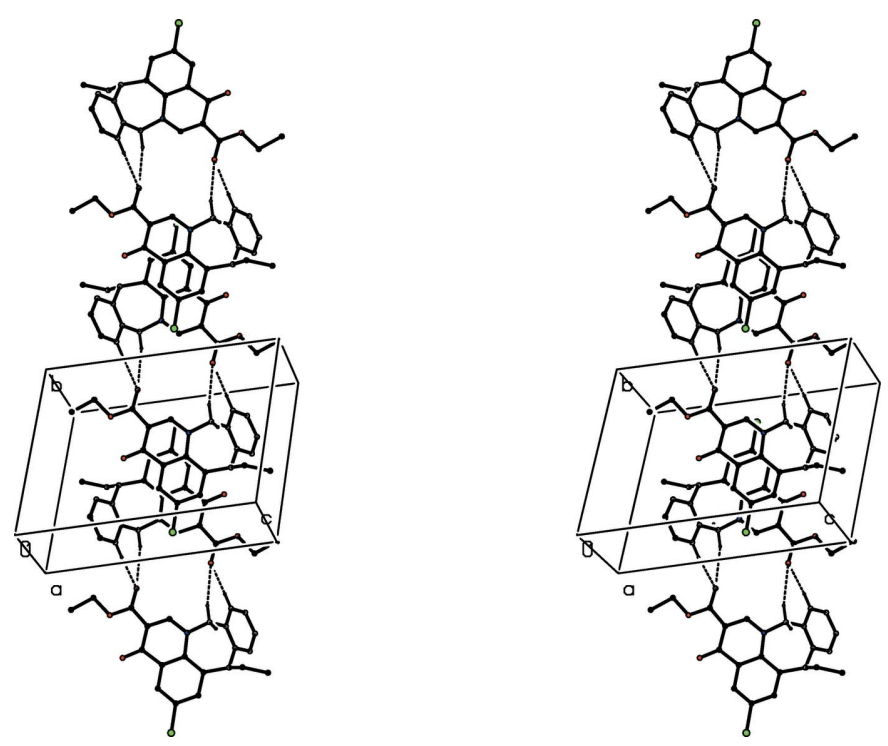

Figure 7

A stereoview of part of the crystal structure of compound (III), showing the formation of a $\pi$-stacked chain of hydrogen-bonded dimers running parallel to the $[1 \overline{1} 0]$ direction. For the sake of clarity, the minor-disorder component and $\mathrm{H}$ atoms bonded to $\mathrm{C}$ atoms that are not involved in the motif shown have been omitted. 
azepine ring as the final step using an $N$-pyrimidoindole precursor for (IV) and an $N$-pyrimidoquinoline precursor for (V). Secondly, the conformation of the azepine ring in compound (IV) differs from the twist-boat form which predominates in (I)-(III) and (V), as this ring contains a significant contribution from the twist-chair form. As a consequence of this, the $C$-methyl group occupies a quasiequatorial position in $(\mathrm{V})$, as expected, but a quasi-axial site in (IV) (Acosta Quintero, Palma et al., 2016). Thirdly, the supramolecular aggregation in the structures of (IV) and (V) differs from that in (I)-(III). The molecules of compound (IV) are linked into $C(5)$ chains by $\mathrm{C}-\mathrm{H} \cdots \mathrm{N}$ hydrogen bonds, although interactions of this type are wholly absent from the structures of (I)-(III), and inversion-related chains of this type are linked into pairs by a $\pi-\pi$ stacking interaction involving the pyrimidine ring. The molecules of compound $(\mathrm{V})$ are linked by $\mathrm{C}-\mathrm{H} \cdots \pi$ (pyrimidine) interactions into cyclic centrosymmetric dimers, somewhat similar to those in the structure of compound (II).

Finally, we note that although the racemic compound (IV) crystallizes in the Sohncke space group $P 2_{1}$, it does so as a conglomerate rather than as a kryptoracemate.

\section{Acknowledgements}

The authors thank 'Centro de Instrumentación CientíficoTécnica of Universidad de Jaén' and Professor Pascal Roussel (University of Lille, France) for data collection. They also thank Vicerrectoría de Investigación y Extensión of Universidad Industrial de Santander (grant No. 9310), the Consejerıa de Innovación, Ciencía y Empresa (Junta de Andalucía, Spain) and the Universidad de Jaén for financial support.

\section{References}

Acosta, L. M., Jurado, J., Palma, A., Cobo, J. \& Glidewell, C. (2015). Acta Cryst. C71, 1062-1068.

Acosta Quintero, L. M., Burgos, I., Palma, A., Cobo, J. \& Glidewell, C. (2016). Acta Cryst. C72, 52-56.

Acosta Quintero, L. M., Jurado, J., Nogueras, M., Palma, A. \& Cobo, J. (2015). Eur. J. Org. Chem. pp. 5360-5369.

Acosta Quintero, L. M., Palma, A., Cobo, J. \& Glidewell, C. (2016). Acta Cryst. C72, 346-357.

Al-Qawasmeh, R. A., Lee, Y., Cao, M.-Y., Gu, X., Viau, S., Lightfoot, J., Wright, J. A. \& Young, A. H. (2009). Bioorg. Med. Chem. Lett. 19, 104-107.
Altomare, A., Cascarano, G., Giacovazzo, C., Guagliardi, A., Burla, M. C., Polidori, G. \& Camalli, M. (1994). J. Appl. Cryst. 27, 435.

Andrés, J. I., Alonso, J. M., Fernández, J., Iturrino, L., Martínez, P., Meert, T. F. \& Sipido, V. K. (2002). Bioorg. Med. Chem. Lett. 12, 3573-3577.

Bernal, I. \& Watkins, S. (2015). Acta Cryst. C71, 216-221.

Bernstein, J., Davis, R. E., Shimoni, L. \& Chang, N.-L. (1995). Angew. Chem. Int. Ed. Engl. 34, 1555-1573.

Bruker (2006). APEX2, SADABS and SAINT. Bruker AXS Inc., Madison, Wisconsin, USA.

Burla, M. C., Caliandro, R., Carrozzini, B., Cascarano, G. L., Cuocci, C., Giacovazzo, C., Mallamo, M., Mazzone, A. \& Polidori, G. (2015). J. Appl. Cryst. 48, 306-309.

Cremer, D. \& Pople, J. A. (1975). J. Am. Chem. Soc. 97, 1354-1358.

Dinesh, N., Kaur, P. K., Swamy, K. K. \& Singh, S. (2014). Exp. Parasitol. 144, 84-90.

Duisenberg, A. J. M., Hooft, R., Schreurs, A. M. M. \& Kroon, J. (2000). J. Appl. Cryst. 33, 893-898.

Duisenberg, A. J. M., Kroon-Batenburg, L. M. J. \& Schreurs, A. M. M. (2003). J. Appl. Cryst. 36, 220-229.

Evans, D. G. \& Boeyens, J. C. A. (1989). Acta Cryst. B45, 581-590.

Fábián, L. \& Brock, C. P. (2010). Acta Cryst. B66, 94-103.

Ferguson, G., Glidewell, C., Gregson, R. M. \& Meehan, P. R. (1998a). Acta Cryst. B54, 129-138.

Ferguson, G., Glidewell, C., Gregson, R. M. \& Meehan, P. R. (1998b). Acta Cryst. B54, 139-150.

Flack, H. D. (1983). Acta Cryst. A39, 876-881.

Gregson, R. M., Glidewell, C., Ferguson, G. \& Lough, A. J. (2000). Acta Cryst. B56, 39-57.

Hooft, R. W. W., Straver, L. H. \& Spek, A. L. (2010). J. Appl. Cryst. 43, 665-668.

Liu, K. K.-C., Li, J. \& Sakya, S. (2004). Mini Rev. Med. Chem. 4, 11051125.

Morales, G. A. \& Fronczek, F. R. (1996). Acta Cryst. C52, 1266-1268.

Mugnaini, C., Pasquini, S. \& Corelli, F. (2009). Curr. Med. Chem. 16, 1746-1767.

Nonius (1998). COLLECT. Nonius BV, Delft, The Netherlands.

Palma, A., Barajas, J. J., Kouznetsov, V. V., Stashenko, E., Bahsas, A. \& Amaro-Luis, J. (2004). Synlett, pp. 2721-2724.

Palma, A., Galeano, N. \& Bahsas, A. (2010). Synthesis, pp. 1291-1302.

Parsons, S., Flack, H. D. \& Wagner, T. (2013). Acta Cryst. B69, 249-259.

Rowland, R. S. \& Taylor, R. (1996). J. Phys. Chem. 100, 7384-7391.

Sanabría, C. M., Palma, A., Cobo, J. \& Glidewell, C. (2014). Acta Cryst. C70, 332-337.

Sheldrick, G. M. (2003). SADABS. University of Göttingen, Germany.

Sheldrick, G. M. (2015). Acta Cryst. C71, 3-8.

Spek, A. L. (2009). Acta Cryst. D65, 148-155.

Stappers, F., Broeckx, R., Leurs, S., Van Den Bergh, L., Agten, J., Lambrechts, A., Van den Heuvel, D. \& De Smaele, D. (2002). Org. Process Res. Dev. 6, 911-914.

Wikström, H. V., Mensonides-Harsema, M. M., Cremers, T. I. F. H., Moltzen, E. K. \& Arnt, J. (2002). J. Med. Chem. 45, 3280-3285. 


\section{supporting information}

Acta Cryst. (2017). C73, 28-35 [https://doi.org/10.1107/S2053229616018143]

Three tetracyclic dibenzoazepine derivatives exhibiting different molecular conformations, different patterns of intermolecular hydrogen bonding and different modes of supramolecular aggregation

Jeferson B. Mateus-Ruíz, Lina M. Acosta Quintero, Alirio Palma, Mario A. Macías, Justo Cobo and Christopher Glidewell

Computing details

Data collection: APEX2 (Bruker, 2006) for (I), (II); COLLECT (Nonius, 1998) for (III). Cell refinement: SAINT (Bruker, 2006) for (I), (II); DIRAX/LSQ (Duisenberg et al., 2000) for (III). Data reduction: SAINT (Bruker, 2006) for (I), (II); EVALCCD (Duisenberg et al., 2003) for (III). Program(s) used to solve structure: SIR92 (Altomare et al., 1994) for (I), (II); SIR2014 (Burla et al., 2015) for (III). For all compounds, program(s) used to refine structure: SHELXL2014 (Sheldrick, 2015); molecular graphics: PLATON (Spek, 2009); software used to prepare material for publication: SHELXL2014 (Sheldrick, 2015) and PLATON (Spek, 2009).

(I) $(9 R S, 15 R S)-9$-Ethyl-11-methyl-9,13b-dihydrodibenzo[c,f] thiazolo[3,2-a]azepin-3(2H)-one

Crystal data

$\mathrm{C}_{19} \mathrm{H}_{19} \mathrm{NOS}$

$M_{r}=309.41$

Monoclinic, $P 2_{1}$

$a=11.4261(5) \AA$

$b=8.1847(3) \AA$

$c=16.8243(6) \AA$

$\beta=93.067(2)^{\circ}$

$V=1571.14(11) \AA^{3}$

$Z=4$

Data collection

Bruker Kappa APEXII diffractometer

Radiation source: high brilliance microfocus sealed tube

$\varphi$ and $\omega$ scans

Absorption correction: multi-scan

(SADABS; Bruker, 2006)

$T_{\min }=0.823, T_{\max }=0.955$

Refinement

Refinement on $F^{2}$

Least-squares matrix: full

$R\left[F^{2}>2 \sigma\left(F^{2}\right)\right]=0.029$
$F(000)=656$

$D_{\mathrm{x}}=1.308 \mathrm{Mg} \mathrm{m}^{-3}$

Mo $K \alpha$ radiation, $\lambda=0.71073 \AA$

Cell parameters from 11002 reflections

$\theta=1.8-32.5^{\circ}$

$\mu=0.21 \mathrm{~mm}^{-1}$

$T=100 \mathrm{~K}$

Block, colourless

$0.40 \times 0.35 \times 0.22 \mathrm{~mm}$

61785 measured reflections 11002 independent reflections 10697 reflections with $I>2 \sigma(I)$

$R_{\text {int }}=0.025$

$\theta_{\max }=32.5^{\circ}, \theta_{\min }=1.8^{\circ}$

$h=-17 \rightarrow 16$

$k=-12 \rightarrow 12$

$l=-25 \rightarrow 25$

$w R\left(F^{2}\right)=0.079$

$S=1.05$

11002 reflections 
401 parameters

1 restraint

Hydrogen site location: inferred from neighbouring sites

$\mathrm{H}$-atom parameters constrained

$w=1 /\left[\sigma^{2}\left(F_{\mathrm{o}}^{2}\right)+(0.0527 P)^{2}+0.1585 P\right]$

where $P=\left(F_{\mathrm{o}}^{2}+2 F_{\mathrm{c}}^{2}\right) / 3$
$(\Delta / \sigma)_{\max }=0.001$

$\Delta \rho_{\max }=0.37$ e $\AA^{-3}$

$\Delta \rho_{\min }=-0.21$ e $\AA^{-3}$

Absolute structure: Flack $x$ determined using 4787 quotients $[(\mathrm{I}+)-(\mathrm{I}-)] /[(\mathrm{I}+)+(\mathrm{I}-)]$ (Parsons et al., 2013)

Absolute structure parameter: 0.007 (6)

Special details

Geometry. All esds (except the esd in the dihedral angle between two 1.s. planes) are estimated using the full covariance matrix. The cell esds are taken into account individually in the estimation of esds in distances, angles and torsion angles; correlations between esds in cell parameters are only used when they are defined by crystal symmetry. An approximate (isotropic) treatment of cell esds is used for estimating esds involving 1.s. planes.

Fractional atomic coordinates and isotropic or equivalent isotropic displacement parameters $\left(\AA^{2}\right)$

\begin{tabular}{|c|c|c|c|c|}
\hline & $x$ & $y$ & $z$ & $U_{\text {iso }} * / U_{\text {eq }}$ \\
\hline $\mathrm{S} 11$ & $0.04257(3)$ & $0.64856(4)$ & $0.31677(2)$ & $0.02072(7)$ \\
\hline $\mathrm{C} 12$ & $-0.06240(11)$ & $0.6542(2)$ & $0.39359(8)$ & $0.0221(2)$ \\
\hline $\mathrm{H} 12 \mathrm{~A}$ & -0.0975 & 0.7643 & 0.3973 & $0.026^{*}$ \\
\hline H12B & -0.1258 & 0.5737 & 0.3825 & $0.026^{*}$ \\
\hline C13 & $0.00808(11)$ & $0.61139(17)$ & $0.46981(8)$ & $0.0198(2)$ \\
\hline O13 & $-0.03273(10)$ & $0.55030(17)$ & $0.52867(7)$ & $0.0298(2)$ \\
\hline N14 & $0.12297(9)$ & $0.65093(16)$ & $0.46388(6)$ & 0.01701 (19) \\
\hline C14A & $0.20563(11)$ & $0.63483(17)$ & $0.52973(7)$ & $0.0170(2)$ \\
\hline $\mathrm{C} 15$ & $0.19005(13)$ & 0.72209 (19) & $0.59932(8)$ & $0.0221(2)$ \\
\hline H15 & 0.1246 & 0.7927 & 0.6032 & $0.027 *$ \\
\hline $\mathrm{C} 16$ & $0.27158(15)$ & $0.7045(2)$ & $0.66311(8)$ & 0.0256 \\
\hline H16 & 0.2618 & 0.7623 & 0.7113 & $0.031 *$ \\
\hline $\mathrm{C} 17$ & $0.36731(14)$ & $0.6021(2)$ & $0.65618(8)$ & $0.0243(3)$ \\
\hline H17 & 0.4241 & 0.5927 & 0.6993 & $0.029 *$ \\
\hline C18 & $0.38124(11)$ & $0.51298(19)$ & $0.58668(7)$ & $0.0195(2)$ \\
\hline $\mathrm{H} 18$ & 0.4466 & 0.4422 & 0.5832 & $0.023 *$ \\
\hline C18A & $0.29966(11)$ & $0.52719(16)$ & $0.52223(7)$ & $0.0158(2)$ \\
\hline C19 & $0.30872(10)$ & $0.43915(16)$ & $0.44299(7)$ & $0.0151(2)$ \\
\hline H19 & 0.2274 & 0.4054 & 0.4252 & $0.018 *$ \\
\hline C19A & $0.34743(11)$ & $0.56585(16)$ & $0.38285(7)$ & $0.0148(2)$ \\
\hline C110 & $0.45748(11)$ & $0.55481(17)$ & $0.35032(7)$ & $0.0164(2)$ \\
\hline H110 & 0.5071 & 0.4655 & 0.3650 & $0.020 *$ \\
\hline C111 & $0.49666(11)$ & $0.67094(17)$ & $0.29695(7)$ & $0.0173(2)$ \\
\hline C112 & $0.42386(11)$ & $0.80170(18)$ & $0.27585(7)$ & $0.0185(2)$ \\
\hline H112 & 0.4482 & 0.8808 & 0.2388 & $0.022 *$ \\
\hline C113 & $0.31535(11)$ & $0.81690(17)$ & $0.30887(7)$ & $0.0177(2)$ \\
\hline H113 & 0.2672 & 0.9083 & 0.2951 & $0.021 *$ \\
\hline C114 & $0.27587(10)$ & $0.70040(16)$ & $0.36181(7)$ & $0.0155(2)$ \\
\hline C115 & $0.15382(11)$ & $0.72903(16)$ & $0.38914(7)$ & $0.0160(2)$ \\
\hline H115 & 0.1415 & 0.8495 & 0.3942 & $0.019 *$ \\
\hline C116 & $0.61535(12)$ & $0.6532(2)$ & $0.26305(8)$ & $0.0246(3)$ \\
\hline H16A & 0.6057 & 0.6325 & 0.2057 & $0.037 *$ \\
\hline
\end{tabular}




\begin{tabular}{|c|c|c|c|c|}
\hline H16B & 0.6572 & 0.5615 & 0.2890 & $0.037^{*}$ \\
\hline $\mathrm{H} 16 \mathrm{C}$ & 0.6603 & 0.7539 & 0.2724 & $0.037^{*}$ \\
\hline C191 & $0.38285(11)$ & $0.28333(17)$ & $0.44701(7)$ & $0.0178(2)$ \\
\hline H19A & 0.3929 & 0.2431 & 0.3923 & $0.021^{*}$ \\
\hline H19B & 0.4615 & 0.3093 & 0.4713 & $0.021 *$ \\
\hline C192 & $0.32763(11)$ & $0.14856(19)$ & $0.49539(8)$ & $0.0211(2)$ \\
\hline $\mathrm{H} 19 \mathrm{C}$ & 0.3245 & 0.1837 & 0.5509 & $0.032 *$ \\
\hline H19D & 0.3749 & 0.0490 & 0.4929 & $0.032 *$ \\
\hline H19E & 0.2480 & 0.1265 & 0.4734 & $0.032 *$ \\
\hline S21 & $0.22593(3)$ & $0.00063(5)$ & $-0.08726(2)$ & $0.02161(7)$ \\
\hline $\mathrm{C} 22$ & $0.06874(13)$ & $0.02911(19)$ & $-0.08922(8)$ & $0.0224(3)$ \\
\hline $\mathrm{H} 22 \mathrm{~A}$ & 0.0435 & 0.1113 & -0.1298 & $0.027^{*}$ \\
\hline H22B & 0.0276 & -0.0750 & -0.1016 & $0.027 *$ \\
\hline C23 & $0.04285(12)$ & $0.08804(18)$ & $-0.00633(8)$ & $0.0205(2)$ \\
\hline $\mathrm{O} 23$ & $-0.04535(10)$ & $0.16254(18)$ & $0.00951(7)$ & $0.0317(3)$ \\
\hline N24 & $0.13069(9)$ & $0.04697(14)$ & $0.04793(6)$ & 0.01589 (19) \\
\hline $\mathrm{C} 24 \mathrm{~A}$ & $0.12426(10)$ & $0.08689(16)$ & $0.13011(7)$ & $0.0151(2)$ \\
\hline C25 & $0.03501(11)$ & $0.02280(18)$ & $0.17405(8)$ & $0.0196(2)$ \\
\hline $\mathrm{H} 25$ & -0.0202 & -0.0517 & 0.1503 & $0.024 *$ \\
\hline $\mathrm{C} 26$ & $0.02786(12)$ & $0.06939(18)$ & $0.25329(9)$ & 0.0219 (2) \\
\hline H26 & -0.0332 & 0.0281 & 0.2838 & $0.026^{*}$ \\
\hline $\mathrm{C} 27$ & $0.11024(12)$ & $0.17634(18)$ & $0.28750(8)$ & 0.0207 (2) \\
\hline $\mathrm{H} 27$ & 0.1057 & 0.2075 & 0.3416 & $0.025^{*}$ \\
\hline $\mathrm{C} 28$ & $0.19976(11)$ & $0.23845(17)$ & $0.24303(7)$ & $0.0175(2)$ \\
\hline $\mathrm{H} 28$ & 0.2562 & 0.3104 & 0.2674 & $0.021^{*}$ \\
\hline $\mathrm{C} 28 \mathrm{~A}$ & $0.20726(10)$ & $0.19610(15)$ & $0.16314(7)$ & $0.0141(2)$ \\
\hline C29 & $0.30020(10)$ & $0.26061(15)$ & $0.10960(7)$ & $0.0140(2)$ \\
\hline $\mathrm{H} 29$ & 0.2589 & 0.2824 & 0.0567 & $0.017 *$ \\
\hline C29A & $0.38728(10)$ & $0.12397(15)$ & $0.09590(7)$ & $0.0140(2)$ \\
\hline $\mathrm{C} 210$ & $0.50569(10)$ & $0.13608(17)$ & $0.12168(7)$ & 0.0157 (2) \\
\hline $\mathrm{H} 210$ & 0.5318 & 0.2316 & 0.1494 & $0.019 *$ \\
\hline $\mathrm{C} 211$ & $0.58688(10)$ & $0.01222(18)$ & $0.10797(7)$ & $0.0170(2)$ \\
\hline C212 & $0.54650(11)$ & $-0.12996(18)$ & $0.06995(7)$ & $0.0183(2)$ \\
\hline $\mathrm{H} 212$ & 0.5995 & -0.2166 & 0.0609 & $0.022 *$ \\
\hline $\mathrm{C} 213$ & $0.42911(11)$ & $-0.14551(17)$ & $0.04519(7)$ & 0.0167 (2) \\
\hline $\mathrm{H} 213$ & 0.4027 & -0.2437 & 0.0201 & $0.020 *$ \\
\hline $\mathrm{C} 214$ & $0.34913(10)$ & $-0.01951(16)$ & $0.05650(7)$ & 0.01384 (19) \\
\hline $\mathrm{C} 215$ & $0.22787(10)$ & $-0.04930(15)$ & $0.01916(7)$ & $0.0144(2)$ \\
\hline $\mathrm{H} 215$ & 0.2088 & -0.1678 & 0.0248 & $0.017^{*}$ \\
\hline $\mathrm{C} 216$ & $0.71526(11)$ & $0.0308(2)$ & $0.13229(8)$ & $0.0233(3)$ \\
\hline $\mathrm{H} 26 \mathrm{~A}$ & 0.7347 & 0.1472 & 0.1370 & $0.035^{*}$ \\
\hline H26B & 0.7625 & -0.0195 & 0.0920 & $0.035^{*}$ \\
\hline $\mathrm{H} 26 \mathrm{C}$ & 0.7319 & -0.0230 & 0.1837 & $0.035^{*}$ \\
\hline C291 & $0.35367(11)$ & $0.42370(16)$ & $0.13740(8)$ & $0.0172(2)$ \\
\hline $\mathrm{H} 29 \mathrm{~A}$ & 0.3887 & 0.4117 & 0.1922 & $0.021 *$ \\
\hline $\mathrm{H} 29 \mathrm{~B}$ & 0.4168 & 0.4547 & 0.1023 & $0.021 *$ \\
\hline C292 & $0.26090(13)$ & $0.55806(18)$ & $0.13589(8)$ & $0.0222(2)$ \\
\hline $\mathrm{H} 29 \mathrm{C}$ & 0.2227 & 0.5652 & 0.0824 & $0.033^{*}$ \\
\hline
\end{tabular}




\begin{tabular}{lllll}
$\mathrm{H} 29 \mathrm{D}$ & 0.2982 & 0.6628 & 0.1498 & $0.033^{*}$ \\
$\mathrm{H} 29 \mathrm{E}$ & 0.2023 & 0.5324 & 0.1745 & $0.033^{*}$ \\
\hline
\end{tabular}

Atomic displacement parameters $\left(\AA^{2}\right)$

\begin{tabular}{|c|c|c|c|c|c|c|}
\hline & $U^{11}$ & $U^{22}$ & $U^{33}$ & $U^{12}$ & $U^{13}$ & $U^{23}$ \\
\hline S11 & $0.01711(13)$ & $0.02520(16)$ & $0.01967(13)$ & $0.00113(12)$ & $-0.00079(10)$ & $-0.00009(12)$ \\
\hline $\mathrm{C} 12$ & $0.0153(5)$ & $0.0253(7)$ & $0.0256(6)$ & $0.0012(5)$ & $0.0011(4)$ & $0.0039(5)$ \\
\hline $\mathrm{C} 13$ & $0.0151(5)$ & $0.0196(6)$ & $0.0251(6)$ & $0.0016(4)$ & $0.0046(4)$ & $0.0031(4)$ \\
\hline $\mathrm{O} 13$ & 0.0208 (5) & $0.0394(7)$ & 0.0298 (5) & $-0.0007(5)$ & $0.0080(4)$ & $0.0110(5)$ \\
\hline N14 & $0.0143(4)$ & $0.0211(5)$ & $0.0160(4)$ & $0.0011(4)$ & 0.0037 (3) & $0.0025(4)$ \\
\hline C14A & $0.0177(5)$ & $0.0183(6)$ & $0.0153(4)$ & $-0.0010(4)$ & $0.0030(4)$ & $0.0013(4)$ \\
\hline C15 & $0.0276(6)$ & $0.0210(6)$ & $0.0183(5)$ & $0.0014(5)$ & $0.0063(5)$ & $-0.0008(5)$ \\
\hline C16 & $0.0371(8)$ & $0.0240(7)$ & 0.0159 (5) & $-0.0034(6)$ & $0.0032(5)$ & $-0.0014(5)$ \\
\hline C17 & $0.0291(7)$ & $0.0283(7)$ & $0.0151(5)$ & $-0.0049(5)$ & $-0.0018(5)$ & $0.0008(5)$ \\
\hline $\mathrm{C} 18$ & $0.0183(5)$ & $0.0243(6)$ & $0.0159(5)$ & $-0.0018(5)$ & $0.0004(4)$ & $0.0033(4)$ \\
\hline C18A & $0.0158(5)$ & $0.0179(6)$ & 0.0139 (4) & -0.0018 & $0.0026(4)$ & 0.0019 (4) \\
\hline C19 & $0.0138(5)$ & $0.0174(5)$ & $0.0142(4)$ & $0.0006(4)$ & $0.0015(4)$ & $0.0014(4)$ \\
\hline C19A & $0.0147(5)$ & $0.0173(5)$ & $0.0126(4)$ & -0.0003 & 0.0019 (4) & $0.0004(4)$ \\
\hline C110 & $0.0152(5)$ & $0.0205(6)$ & $0.0137(4)$ & $0.0000(4)$ & $0.0020(4)$ & $0.0001(4)$ \\
\hline C111 & $0.0160(5)$ & $0.0225(6)$ & $0.0136(4)$ & $-0.0019(4)$ & $0.0028(4)$ & $0.0001(4)$ \\
\hline C112 & $0.0192(5)$ & $0.0220(6)$ & $0.0145(5)$ & $-0.0024(5)$ & $0.0020(4)$ & 0.0028 (4) \\
\hline $\mathrm{C} 113$ & $0.0182(5)$ & $0.0186(6)$ & $0.0162(5)$ & -0.0007 (4) & $0.0013(4)$ & $0.0028(4)$ \\
\hline C114 & $0.0143(5)$ & $0.0180(5)$ & $0.0143(4)$ & $-0.0004(4)$ & $0.0021(4)$ & $0.0003(4)$ \\
\hline C115 & $0.0153(5)$ & $0.0169(5)$ & $0.0159(5)$ & $0.0003(4)$ & $0.0026(4)$ & $0.0014(4)$ \\
\hline C116 & $0.0188(5)$ & $0.0323(7)$ & $0.0234(6)$ & $0.0010(6)$ & $0.0087(4)$ & $0.0042(5)$ \\
\hline C191 & $0.0158(5)$ & $0.0195(6)$ & $0.0181(5)$ & $0.0020(4)$ & $0.0025(4)$ & $0.0030(4)$ \\
\hline C192 & $0.0181(5)$ & $0.0204(6)$ & $0.0250(5)$ & $0.0006(5)$ & $0.0023(4)$ & $0.0051(5)$ \\
\hline S21 & $0.02265(14)$ & $0.02804(18)$ & $0.01408(12)$ & $-0.00534(12)$ & $0.00057(10)$ & $-0.00045(11)$ \\
\hline $\mathrm{C} 22$ & $0.0241(6)$ & $0.0233(7)$ & $0.0189(5)$ & $0.0003(5)$ & $-0.0065(4)$ & $-0.0005(5)$ \\
\hline $\mathrm{C} 23$ & $0.0192(5)$ & $0.0192(6)$ & $0.0223(5)$ & $0.0020(5)$ & $-0.0061(4)$ & $-0.0026(5)$ \\
\hline $\mathrm{O} 23$ & $0.0241(5)$ & $0.0360(6)$ & $0.0339(5)$ & $0.0138(5)$ & $-0.0088(4)$ & $-0.0078(5)$ \\
\hline N24 & $0.0131(4)$ & $0.0182(5)$ & $0.0162(4)$ & $0.0020(4)$ & $-0.0013(3)$ & $-0.0022(4)$ \\
\hline $\mathrm{C} 24 \mathrm{~A}$ & $0.0133(5)$ & $0.0158(5)$ & $0.0164(5)$ & $0.0011(4)$ & $0.0010(4)$ & $-0.0006(4)$ \\
\hline $\mathrm{C} 25$ & $0.0141(5)$ & $0.0205(6)$ & $0.0245(5)$ & -0.0009 (4) & $0.0041(4)$ & $-0.0009(5)$ \\
\hline $\mathrm{C} 26$ & $0.0197(6)$ & $0.0215(6)$ & $0.0253(6)$ & $0.0009(5)$ & $0.0093(5)$ & $0.0024(5)$ \\
\hline $\mathrm{C} 27$ & $0.0238(6)$ & $0.0210(6)$ & $0.0180(5)$ & $0.0034(5)$ & 0.0062 (4) & $0.0004(4)$ \\
\hline $\mathrm{C} 28$ & $0.0199(5)$ & $0.0174(6)$ & $0.0155(5)$ & 0.0009 (4) & $0.0016(4)$ & $-0.0006(4)$ \\
\hline C28A & 0.0133 (4) & $0.0142(5)$ & $0.0150(4)$ & $0.0012(4)$ & 0.0014 (4) & $-0.0002(4)$ \\
\hline C29 & $0.0149(5)$ & $0.0132(5)$ & $0.0140(4)$ & -0.0013 & 0.0012 (4) & $-0.0002(4)$ \\
\hline C29A & 0.0132 (4) & $0.0152(5)$ & 0.0138 (4) & $-0.0006(4)$ & $0.0021(3)$ & $0.0012(4)$ \\
\hline $\mathrm{C} 210$ & 0.0149 (4) & $0.0183(5)$ & $0.0140(4)$ & -0.0023 & $0.0016(3)$ & 0.0014 (4) \\
\hline $\mathrm{C} 211$ & 0.0134 (4) & $0.0232(6)$ & 0.0145 (4) & $0.0002(4)$ & 0.0023 (4) & $0.0034(4)$ \\
\hline $\mathrm{C} 212$ & $0.0159(5)$ & $0.0226(6)$ & $0.0167(5)$ & $0.0034(4)$ & $0.0037(4)$ & 0.0007 (4) \\
\hline $\mathrm{C} 213$ & $0.0171(5)$ & $0.0175(6)$ & $0.0159(5)$ & $0.0013(4)$ & $0.0032(4)$ & -0.0010 \\
\hline $\mathrm{C} 214$ & $0.0133(4)$ & $0.0148(5)$ & $0.0136(4)$ & -0.0005 & $0.0019(3)$ & $0.0000(4)$ \\
\hline $\mathrm{C} 215$ & $0.0139(5)$ & $0.0149(5)$ & 0.0144 (4) & $0.0001(4)$ & 0.0008 (4) & -0.0009 \\
\hline $\mathrm{C} 216$ & $0.0131(5)$ & $0.0336(8)$ & $0.0231(6)$ & $0.0003(5)$ & $0.0002(4)$ & $0.0015(5)$ \\
\hline
\end{tabular}




\begin{tabular}{lllllll}
$\mathrm{C} 291$ & $0.0190(5)$ & $0.0149(5)$ & $0.0178(5)$ & $-0.0027(4)$ & $-0.0001(4)$ & $-0.0013(4)$ \\
$\mathrm{C} 292$ & $0.0274(6)$ & $0.0155(6)$ & $0.0235(6)$ & $0.0006(5)$ & $0.0005(5)$ & $-0.0005(4)$ \\
\hline
\end{tabular}

Geometric parameters $(\AA, \stackrel{\circ}{)}$

\begin{tabular}{|c|c|c|c|}
\hline $\mathrm{S} 11-\mathrm{C} 12$ & $1.8102(14)$ & $\mathrm{S} 21-\mathrm{C} 22$ & $1.8096(15)$ \\
\hline $\mathrm{S} 11-\mathrm{C} 115$ & $1.8348(13)$ & $\mathrm{S} 21-\mathrm{C} 215$ & $1.8354(12)$ \\
\hline $\mathrm{C} 12-\mathrm{C} 13$ & $1.5184(19)$ & $\mathrm{C} 22-\mathrm{C} 23$ & $1.520(2)$ \\
\hline $\mathrm{C} 12-\mathrm{H} 12 \mathrm{~A}$ & 0.9900 & $\mathrm{C} 22-\mathrm{H} 22 \mathrm{~A}$ & 0.9900 \\
\hline $\mathrm{C} 12-\mathrm{H} 12 \mathrm{~B}$ & 0.9900 & $\mathrm{C} 22-\mathrm{H} 22 \mathrm{~B}$ & 0.9900 \\
\hline $\mathrm{C} 13-\mathrm{O} 13$ & $1.2238(16)$ & $\mathrm{C} 23-\mathrm{O} 23$ & $1.2196(17)$ \\
\hline $\mathrm{C} 13-\mathrm{N} 14$ & $1.3609(16)$ & $\mathrm{C} 23-\mathrm{N} 24$ & $1.3623(16)$ \\
\hline $\mathrm{N} 14-\mathrm{C} 14 \mathrm{~A}$ & $1.4229(15)$ & $\mathrm{N} 24-\mathrm{C} 24 \mathrm{~A}$ & $1.4264(16)$ \\
\hline N14-C115 & $1.4702(16)$ & $\mathrm{N} 24-\mathrm{C} 215$ & $1.4648(16)$ \\
\hline $\mathrm{C} 14 \mathrm{~A}-\mathrm{C} 15$ & $1.3911(18)$ & $\mathrm{C} 24 \mathrm{~A}-\mathrm{C} 25$ & $1.3937(17)$ \\
\hline $\mathrm{C} 14 \mathrm{~A}-\mathrm{C} 18 \mathrm{~A}$ & $1.4003(18)$ & $\mathrm{C} 24 \mathrm{~A}-\mathrm{C} 28 \mathrm{~A}$ & $1.3968(17)$ \\
\hline $\mathrm{C} 15-\mathrm{C} 16$ & $1.390(2)$ & $\mathrm{C} 25-\mathrm{C} 26$ & 1.3933 (19) \\
\hline C15-H15 & 0.9500 & $\mathrm{C} 25-\mathrm{H} 25$ & 0.9500 \\
\hline $\mathrm{C} 16-\mathrm{C} 17$ & $1.387(2)$ & $\mathrm{C} 26-\mathrm{C} 27$ & $1.388(2)$ \\
\hline $\mathrm{C} 16-\mathrm{H} 16$ & 0.9500 & $\mathrm{C} 26-\mathrm{H} 26$ & 0.9500 \\
\hline $\mathrm{C} 17-\mathrm{C} 18$ & $1.3947(19)$ & $\mathrm{C} 27-\mathrm{C} 28$ & $1.3951(18)$ \\
\hline C17-H17 & 0.9500 & $\mathrm{C} 27-\mathrm{H} 27$ & 0.9500 \\
\hline $\mathrm{C} 18-\mathrm{C} 18 \mathrm{~A}$ & $1.3964(17)$ & $\mathrm{C} 28-\mathrm{C} 28 \mathrm{~A}$ & $1.3951(16)$ \\
\hline C18-H18 & 0.9500 & $\mathrm{C} 28-\mathrm{H} 28$ & 0.9500 \\
\hline $\mathrm{C} 18 \mathrm{~A}-\mathrm{C} 19$ & $1.5238(17)$ & $\mathrm{C} 28 \mathrm{~A}-\mathrm{C} 29$ & $1.5232(17)$ \\
\hline C19-C191 & $1.5305(18)$ & $\mathrm{C} 29-\mathrm{C} 29 \mathrm{~A}$ & $1.5226(17)$ \\
\hline C19-C19A & $1.5306(17)$ & C29-C291 & $1.5308(17)$ \\
\hline C19-H19 & 1.0000 & $\mathrm{C} 29-\mathrm{H} 29$ & 1.0000 \\
\hline $\mathrm{C} 19 \mathrm{~A}-\mathrm{C} 110$ & $1.4009(16)$ & $\mathrm{C} 29 \mathrm{~A}-\mathrm{C} 210$ & $1.4020(16)$ \\
\hline $\mathrm{C} 19 \mathrm{~A}-\mathrm{C} 114$ & $1.4056(18)$ & $\mathrm{C} 29 \mathrm{~A}-\mathrm{C} 214$ & $1.4063(17)$ \\
\hline $\mathrm{C} 110-\mathrm{C} 111$ & $1.3975(17)$ & $\mathrm{C} 210-\mathrm{C} 211$ & $1.4015(18)$ \\
\hline $\mathrm{C} 110-\mathrm{H} 110$ & 0.9500 & $\mathrm{C} 210-\mathrm{H} 210$ & 0.9500 \\
\hline $\mathrm{C} 111-\mathrm{C} 112$ & $1.3899(19)$ & $\mathrm{C} 211-\mathrm{C} 212$ & $1.3946(19)$ \\
\hline C111—C116 & $1.5061(18)$ & C211-C216 & $1.5094(17)$ \\
\hline $\mathrm{C} 112-\mathrm{C} 113$ & $1.3911(17)$ & $\mathrm{C} 212-\mathrm{C} 213$ & $1.3890(18)$ \\
\hline C112-H112 & 0.9500 & $\mathrm{C} 212-\mathrm{H} 212$ & 0.9500 \\
\hline C113-C114 & $1.3960(17)$ & $\mathrm{C} 213-\mathrm{C} 214$ & $1.3976(17)$ \\
\hline C113-H113 & 0.9500 & $\mathrm{C} 213-\mathrm{H} 213$ & 0.9500 \\
\hline C114-C115 & $1.5098(17)$ & $\mathrm{C} 214-\mathrm{C} 215$ & $1.5102(16)$ \\
\hline C115-H115 & 1.0000 & $\mathrm{C} 215-\mathrm{H} 215$ & 1.0000 \\
\hline $\mathrm{C} 116-\mathrm{H} 16 \mathrm{~A}$ & 0.9800 & $\mathrm{C} 216-\mathrm{H} 26 \mathrm{~A}$ & 0.9800 \\
\hline C116-H16B & 0.9800 & $\mathrm{C} 216-\mathrm{H} 26 \mathrm{~B}$ & 0.9800 \\
\hline $\mathrm{C} 116-\mathrm{H} 16 \mathrm{C}$ & 0.9800 & $\mathrm{C} 216-\mathrm{H} 26 \mathrm{C}$ & 0.9800 \\
\hline C191-C192 & $1.5271(19)$ & C291-C292 & $1.527(2)$ \\
\hline C191-H19A & 0.9900 & C291- H29A & 0.9900 \\
\hline C191-H19B & 0.9900 & C291- H29B & 0.9900 \\
\hline C192-H19C & 0.9800 & C292-H29C & 0.9800 \\
\hline C192-H19D & 0.9800 & $\mathrm{C} 292-\mathrm{H} 29 \mathrm{D}$ & 0.9800 \\
\hline
\end{tabular}




\begin{tabular}{|c|c|c|c|}
\hline C192-H19E & 0.9800 & $\mathrm{C} 292-\mathrm{H} 29 \mathrm{E}$ & 0.9800 \\
\hline $\mathrm{C} 12-\mathrm{S} 11-\mathrm{C} 115$ & $88.80(6)$ & $\mathrm{C} 22-\mathrm{S} 21-\mathrm{C} 215$ & $90.38(6)$ \\
\hline $\mathrm{C} 13-\mathrm{C} 12-\mathrm{S} 11$ & $104.79(9)$ & $\mathrm{C} 23-\mathrm{C} 22-\mathrm{S} 21$ & $105.42(9)$ \\
\hline $\mathrm{C} 13-\mathrm{C} 12-\mathrm{H} 12 \mathrm{~A}$ & 110.8 & $\mathrm{C} 23-\mathrm{C} 22-\mathrm{H} 22 \mathrm{~A}$ & 110.7 \\
\hline $\mathrm{S} 11-\mathrm{C} 12-\mathrm{H} 12 \mathrm{~A}$ & 110.8 & $\mathrm{~S} 21-\mathrm{C} 22-\mathrm{H} 22 \mathrm{~A}$ & 110.7 \\
\hline $\mathrm{C} 13-\mathrm{C} 12-\mathrm{H} 12 \mathrm{~B}$ & 110.8 & $\mathrm{C} 23-\mathrm{C} 22-\mathrm{H} 22 \mathrm{~B}$ & 110.7 \\
\hline $\mathrm{S} 11-\mathrm{C} 12-\mathrm{H} 12 \mathrm{~B}$ & 110.8 & $\mathrm{~S} 21-\mathrm{C} 22-\mathrm{H} 22 \mathrm{~B}$ & 110.7 \\
\hline $\mathrm{H} 12 \mathrm{~A}-\mathrm{C} 12-\mathrm{H} 12 \mathrm{~B}$ & 108.9 & $\mathrm{H} 22 \mathrm{~A}-\mathrm{C} 22-\mathrm{H} 22 \mathrm{~B}$ & 108.8 \\
\hline $\mathrm{O} 13-\mathrm{C} 13-\mathrm{N} 14$ & $124.56(13)$ & $\mathrm{O} 23-\mathrm{C} 23-\mathrm{N} 24$ & $124.38(13)$ \\
\hline $\mathrm{O} 13-\mathrm{C} 13-\mathrm{C} 12$ & $124.74(12)$ & $\mathrm{O} 23-\mathrm{C} 23-\mathrm{C} 22$ & $124.26(12)$ \\
\hline $\mathrm{N} 14-\mathrm{C} 13-\mathrm{C} 12$ & $110.69(11)$ & $\mathrm{N} 24-\mathrm{C} 23-\mathrm{C} 22$ & $111.36(12)$ \\
\hline $\mathrm{C} 13-\mathrm{N} 14-\mathrm{C} 14 \mathrm{~A}$ & $121.57(11)$ & $\mathrm{C} 23-\mathrm{N} 24-\mathrm{C} 24 \mathrm{~A}$ & $121.25(11)$ \\
\hline $\mathrm{C} 13-\mathrm{N} 14-\mathrm{C} 115$ & $116.26(10)$ & $\mathrm{C} 23-\mathrm{N} 24-\mathrm{C} 215$ & $117.10(10)$ \\
\hline $\mathrm{C} 14 \mathrm{~A}-\mathrm{N} 14-\mathrm{C} 115$ & $121.78(10)$ & $\mathrm{C} 24 \mathrm{~A}-\mathrm{N} 24-\mathrm{C} 215$ & $121.52(10)$ \\
\hline $\mathrm{C} 15-\mathrm{C} 14 \mathrm{~A}-\mathrm{C} 18 \mathrm{~A}$ & $122.15(12)$ & $\mathrm{C} 25-\mathrm{C} 24 \mathrm{~A}-\mathrm{C} 28 \mathrm{~A}$ & $122.04(11)$ \\
\hline $\mathrm{C} 15-\mathrm{C} 14 \mathrm{~A}-\mathrm{N} 14$ & $119.88(12)$ & $\mathrm{C} 25-\mathrm{C} 24 \mathrm{~A}-\mathrm{N} 24$ & $120.33(11)$ \\
\hline $\mathrm{C} 18 \mathrm{~A}-\mathrm{C} 14 \mathrm{~A}-\mathrm{N} 14$ & $117.95(11)$ & $\mathrm{C} 28 \mathrm{~A}-\mathrm{C} 24 \mathrm{~A}-\mathrm{N} 24$ & $117.59(11)$ \\
\hline $\mathrm{C} 16-\mathrm{C} 15-\mathrm{C} 14 \mathrm{~A}$ & $119.06(14)$ & $\mathrm{C} 26-\mathrm{C} 25-\mathrm{C} 24 \mathrm{~A}$ & $119.11(12)$ \\
\hline $\mathrm{C} 16-\mathrm{C} 15-\mathrm{H} 15$ & 120.5 & $\mathrm{C} 26-\mathrm{C} 25-\mathrm{H} 25$ & 120.4 \\
\hline $\mathrm{C} 14 \mathrm{~A}-\mathrm{C} 15-\mathrm{H} 15$ & 120.5 & $\mathrm{C} 24 \mathrm{~A}-\mathrm{C} 25-\mathrm{H} 25$ & 120.4 \\
\hline $\mathrm{C} 17-\mathrm{C} 16-\mathrm{C} 15$ & $119.77(13)$ & $\mathrm{C} 27-\mathrm{C} 26-\mathrm{C} 25$ & $119.76(12)$ \\
\hline $\mathrm{C} 17-\mathrm{C} 16-\mathrm{H} 16$ & 120.1 & $\mathrm{C} 27-\mathrm{C} 26-\mathrm{H} 26$ & 120.1 \\
\hline $\mathrm{C} 15-\mathrm{C} 16-\mathrm{H} 16$ & 120.1 & $\mathrm{C} 25-\mathrm{C} 26-\mathrm{H} 26$ & 120.1 \\
\hline $\mathrm{C} 16-\mathrm{C} 17-\mathrm{C} 18$ & $120.80(13)$ & $\mathrm{C} 26-\mathrm{C} 27-\mathrm{C} 28$ & $120.53(12)$ \\
\hline $\mathrm{C} 16-\mathrm{C} 17-\mathrm{H} 17$ & 119.6 & $\mathrm{C} 26-\mathrm{C} 27-\mathrm{H} 27$ & 119.7 \\
\hline $\mathrm{C} 18-\mathrm{C} 17-\mathrm{H} 17$ & 119.6 & $\mathrm{C} 28-\mathrm{C} 27-\mathrm{H} 27$ & 119.7 \\
\hline $\mathrm{C} 17-\mathrm{C} 18-\mathrm{C} 18 \mathrm{~A}$ & $120.40(13)$ & $\mathrm{C} 27-\mathrm{C} 28-\mathrm{C} 28 \mathrm{~A}$ & $120.72(12)$ \\
\hline $\mathrm{C} 17-\mathrm{C} 18-\mathrm{H} 18$ & 119.8 & $\mathrm{C} 27-\mathrm{C} 28-\mathrm{H} 28$ & 119.6 \\
\hline $\mathrm{C} 18 \mathrm{~A}-\mathrm{C} 18-\mathrm{H} 18$ & 119.8 & $\mathrm{C} 28 \mathrm{~A}-\mathrm{C} 28-\mathrm{H} 28$ & 119.6 \\
\hline $\mathrm{C} 18-\mathrm{C} 18 \mathrm{~A}-\mathrm{C} 14 \mathrm{~A}$ & $117.78(12)$ & $\mathrm{C} 28-\mathrm{C} 28 \mathrm{~A}-\mathrm{C} 24 \mathrm{~A}$ & $117.83(11)$ \\
\hline $\mathrm{C} 18-\mathrm{C} 18 \mathrm{~A}-\mathrm{C} 19$ & $124.48(12)$ & $\mathrm{C} 28-\mathrm{C} 28 \mathrm{~A}-\mathrm{C} 29$ & $124.36(11)$ \\
\hline $\mathrm{C} 14 \mathrm{~A}-\mathrm{C} 18 \mathrm{~A}-\mathrm{C} 19$ & $117.69(11)$ & $\mathrm{C} 24 \mathrm{~A}-\mathrm{C} 28 \mathrm{~A}-\mathrm{C} 29$ & $117.81(10)$ \\
\hline $\mathrm{C} 18 \mathrm{~A}-\mathrm{C} 19-\mathrm{C} 191$ & $114.75(10)$ & $\mathrm{C} 29 \mathrm{~A}-\mathrm{C} 29-\mathrm{C} 28 \mathrm{~A}$ & $108.47(10)$ \\
\hline $\mathrm{C} 18 \mathrm{~A}-\mathrm{C} 19-\mathrm{C} 19 \mathrm{~A}$ & $107.07(10)$ & $\mathrm{C} 29 \mathrm{~A}-\mathrm{C} 29-\mathrm{C} 291$ & $115.68(10)$ \\
\hline $\mathrm{C} 191-\mathrm{C} 19-\mathrm{C} 19 \mathrm{~A}$ & $114.52(10)$ & $\mathrm{C} 28 \mathrm{~A}-\mathrm{C} 29-\mathrm{C} 291$ & $113.64(10)$ \\
\hline $\mathrm{C} 18 \mathrm{~A}-\mathrm{C} 19-\mathrm{H} 19$ & 106.6 & $\mathrm{C} 29 \mathrm{~A}-\mathrm{C} 29-\mathrm{H} 29$ & 106.1 \\
\hline C191-C19-H19 & 106.6 & $\mathrm{C} 28 \mathrm{~A}-\mathrm{C} 29-\mathrm{H} 29$ & 106.1 \\
\hline C19A-C19-H19 & 106.6 & $\mathrm{C} 291-\mathrm{C} 29-\mathrm{H} 29$ & 106.1 \\
\hline $\mathrm{C} 110-\mathrm{C} 19 \mathrm{~A}-\mathrm{C} 114$ & $118.32(11)$ & $\mathrm{C} 210-\mathrm{C} 29 \mathrm{~A}-\mathrm{C} 214$ & $118.33(11)$ \\
\hline $\mathrm{C} 110-\mathrm{C} 19 \mathrm{~A}-\mathrm{C} 19$ & $120.78(11)$ & $\mathrm{C} 210-\mathrm{C} 29 \mathrm{~A}-\mathrm{C} 29$ & $121.93(11)$ \\
\hline $\mathrm{C} 114-\mathrm{C} 19 \mathrm{~A}-\mathrm{C} 19$ & $120.83(10)$ & $\mathrm{C} 214-\mathrm{C} 29 \mathrm{~A}-\mathrm{C} 29$ & $119.74(10)$ \\
\hline $\mathrm{C} 111-\mathrm{C} 110-\mathrm{C} 19 \mathrm{~A}$ & $122.16(12)$ & $\mathrm{C} 211-\mathrm{C} 210-\mathrm{C} 29 \mathrm{~A}$ & $122.29(12)$ \\
\hline $\mathrm{C} 111-\mathrm{C} 110-\mathrm{H} 110$ & 118.9 & $\mathrm{C} 211-\mathrm{C} 210-\mathrm{H} 210$ & 118.9 \\
\hline $\mathrm{C} 19 \mathrm{~A}-\mathrm{C} 110-\mathrm{H} 110$ & 118.9 & $\mathrm{C} 29 \mathrm{~A}-\mathrm{C} 210-\mathrm{H} 210$ & 118.9 \\
\hline $\mathrm{C} 112-\mathrm{C} 111-\mathrm{C} 110$ & $118.68(11)$ & $\mathrm{C} 212-\mathrm{C} 211-\mathrm{C} 210$ & $118.26(11)$ \\
\hline $\mathrm{C} 112-\mathrm{C} 111-\mathrm{C} 116$ & $121.09(12)$ & $\mathrm{C} 212-\mathrm{C} 211-\mathrm{C} 216$ & $120.09(12)$ \\
\hline $\mathrm{C} 110-\mathrm{C} 111-\mathrm{C} 116$ & $120.23(12)$ & $\mathrm{C} 210-\mathrm{C} 211-\mathrm{C} 216$ & $121.64(12)$ \\
\hline
\end{tabular}




$\begin{array}{ll}\text { C111-C112-C113 } & 120.06(12) \\ \text { C111-C112-H112 } & 120.0 \\ \text { C113-C112-H112 } & 120.0 \\ \text { C112-C113-C114 } & 121.27(12) \\ \text { C112-C113-H113 } & 119.4 \\ \text { C114-C113-H113 } & 119.4 \\ \text { C113-C114-C19A } & 119.49(11) \\ \text { C113-C114-C115 } & 115.06(11) \\ \text { C19A-C114-C115 } & 125.39(11) \\ \text { N14-C115-C114 } & 117.43(10) \\ \text { N14-C115-S11 } & 102.84(8) \\ \text { C114-C115-S11 } & 111.14(8) \\ \text { N14-C115-H115 } & 108.4 \\ \text { C114-C115-H115 } & 108.4 \\ \text { S11-C115-H115 } & 108.4 \\ \text { C111-C116-H16A } & 109.5 \\ \text { C111-C116-H16B } & 109.5 \\ \text { H16A-C116-H16B } & 109.5 \\ \text { C111-C116-H16C } & 109.5 \\ \text { H16A-C116-H16C } & 109.5 \\ \text { H16B-C116-H16C } & 109.5 \\ \text { C192-C191-C19 } & 112.46(10) \\ \text { C192-C191-H19A } & 109.1 \\ \text { C19-C191-H19A } & 109.1 \\ \text { C192-C191-H19B } & 109.1 \\ \text { C19-C191-H19B } & 109.1 \\ \text { H19A-C191-H19B } & 107.8 \\ \text { C191-C192-H19C } & 109.5 \\ \text { C191-C192-H19D } & 109.5 \\ \text { H19C-C192-H19D } & 109.5 \\ \text { C191-C192-H19E } & 109.5 \\ \text { H19C-C192-H19E } & 109.5 \\ \text { H19D-C192-H19E } & 109.5 \\ \text { C115-S11-C12-C13 } & -35.23(10) \\ \text { S11-C12-C13-O13 } & -154.57(14) \\ \text { S11-C12-C13-N14 } & 25.70(15) \\ \text { O13-C13-N14-C14A } & -4.8(2) \\ \text { C12-C13-N14-C14A } & 174.94(13) \\ \text { O13-C13-N14-C115 } & -177.78(14) \\ \text { C12-C13-N14-C115 } & 1.96(18) \\ \text { C13-N14-C14A-C15 } & -60.13(19) \\ \text { C115-N14-C14A-C15 } & 112.47(15) \\ \text { C13-N14-C14A-C18A } & 118.52(14) \\ \text { C115-N14-C14A-C18A } & -68.89(17) \\ \text { C18A-C14A-C15-C16 } & 1.2(2) \\ \text { N14-C14A-C15-C16 } & 179.83(13) \\ \text { C14A-C15-C16-C17 } & 0.7(2) \\ & \end{array}$

$\mathrm{C} 213-\mathrm{C} 212-\mathrm{C} 211$

$\mathrm{C} 213-\mathrm{C} 212-\mathrm{H} 212$

$\mathrm{C} 211-\mathrm{C} 212-\mathrm{H} 212$

C212-C213-C214

C212- $2213-\mathrm{H} 213$

$\mathrm{C} 214-\mathrm{C} 213-\mathrm{H} 213$

$\mathrm{C} 213-\mathrm{C} 214-\mathrm{C} 29 \mathrm{~A}$

$\mathrm{C} 213-\mathrm{C} 214-\mathrm{C} 215$

$\mathrm{C} 29 \mathrm{~A}-\mathrm{C} 214-\mathrm{C} 215$

$\mathrm{N} 24-\mathrm{C} 215-\mathrm{C} 214$

$\mathrm{N} 24-\mathrm{C} 215-\mathrm{S} 21$

$\mathrm{C} 214-\mathrm{C} 215-\mathrm{S} 21$

N24-C215-H215

C214-C215-H215

$\mathrm{S} 21-\mathrm{C} 215-\mathrm{H} 215$

C211-C216-H26A

C211-C216- $\mathrm{H} 26 \mathrm{~B}$

$\mathrm{H} 26 \mathrm{~A}-\mathrm{C} 216-\mathrm{H} 26 \mathrm{~B}$

$\mathrm{C} 211-\mathrm{C} 216-\mathrm{H} 26 \mathrm{C}$

$\mathrm{H} 26 \mathrm{~A}-\mathrm{C} 216-\mathrm{H} 26 \mathrm{C}$

$\mathrm{H} 26 \mathrm{~B}-\mathrm{C} 216-\mathrm{H} 26 \mathrm{C}$

C292-C291-C29

C292-C291-H29A

$\mathrm{C} 29-\mathrm{C} 291-\mathrm{H} 29 \mathrm{~A}$

C292-C291-H29B

C29-C291-H29B

H29A-C291-H29B

$\mathrm{C} 291-\mathrm{C} 292-\mathrm{H} 29 \mathrm{C}$

C291-C292-H29D

$\mathrm{H} 29 \mathrm{C}-\mathrm{C} 292-\mathrm{H} 29 \mathrm{D}$

$\mathrm{C} 291-\mathrm{C} 292-\mathrm{H} 29 \mathrm{E}$

$\mathrm{H} 29 \mathrm{C}-\mathrm{C} 292-\mathrm{H} 29 \mathrm{E}$

$\mathrm{H} 29 \mathrm{D}-\mathrm{C} 292-\mathrm{H} 29 \mathrm{E}$

$\mathrm{C} 215-\mathrm{S} 21-\mathrm{C} 22-\mathrm{C} 23$

$\mathrm{S} 21-\mathrm{C} 22-\mathrm{C} 23-\mathrm{O} 23$

$\mathrm{S} 21-\mathrm{C} 22-\mathrm{C} 23-\mathrm{N} 24$

$\mathrm{O} 23-\mathrm{C} 23-\mathrm{N} 24-\mathrm{C} 24 \mathrm{~A}$

$\mathrm{C} 22-\mathrm{C} 23-\mathrm{N} 24-\mathrm{C} 24 \mathrm{~A}$

$\mathrm{O} 23-\mathrm{C} 23-\mathrm{N} 24-\mathrm{C} 215$

$\mathrm{C} 22-\mathrm{C} 23-\mathrm{N} 24-\mathrm{C} 215$

$\mathrm{C} 23-\mathrm{N} 24-\mathrm{C} 24 \mathrm{~A}-\mathrm{C} 25$

C215-N24-C24A-C25

$\mathrm{C} 23-\mathrm{N} 24-\mathrm{C} 24 \mathrm{~A}-\mathrm{C} 28 \mathrm{~A}$

$\mathrm{C} 215-\mathrm{N} 24-\mathrm{C} 24 \mathrm{~A}-\mathrm{C} 28 \mathrm{~A}$

$\mathrm{C} 28 \mathrm{~A}-\mathrm{C} 24 \mathrm{~A}-\mathrm{C} 25-\mathrm{C} 26$

$\mathrm{N} 24-\mathrm{C} 24 \mathrm{~A}-\mathrm{C} 25-\mathrm{C} 26$

$\mathrm{C} 24 \mathrm{~A}-\mathrm{C} 25-\mathrm{C} 26-\mathrm{C} 27$
$120.30(12)$

119.9

119.9

$121.30(12)$

119.3

119.3

$119.47(11)$

$114.60(11)$

$125.82(11)$

$118.07(10)$

$103.46(8)$

109.44 (8)

108.5

108.5

108.5

109.5

109.5

109.5

109.5

109.5

109.5

$110.95(10)$

109.4

109.4

109.4

109.4

108.0

109.5

109.5

109.5

109.5

109.5

109.5

30.15 (10)

$158.58(14)$

$-21.43(15)$

1.3 (2)

$-178.71(12)$

177.26 (14)

$-2.73(17)$

61.92 (18)

$-113.89(13)$

$-115.71(14)$

68.49 (16)

0.3 (2)

$-177.25(12)$

-1.0 (2) 


$\begin{array}{ll}\text { C15-C16-C17-C18 } & -1.8(2) \\ \text { C16-C17-C18-C18A } & 1.0(2) \\ \text { C17-C18-C18A-C14A } & 0.90(19) \\ \text { C17-C18-C18A-C19 } & 178.15(13) \\ \text { C15-C14A-C18A-C18 } & -2.03(19) \\ \text { N14-C14A-C18A-C18 } & 179.36(12) \\ \text { C15-C14A-C18A-C19 } & -179.46(12) \\ \text { N14-C14A-C18A-C19 } & 1.92(17) \\ \text { C18-C18A-C19-C191 } & 23.65(17) \\ \text { C14A-C18A-C19-C191 } & -159.10(11) \\ \text { C18-C18A-C19-C19A } & -104.63(14) \\ \text { C14A-C18A-C19-C19A } & 72.62(13) \\ \text { C18A-C19-C19A-C110 } & 113.42(12) \\ \text { C191-C19-C19A-C110 } & -15.00(16) \\ \text { C18A-C19-C19A-C114 } & -63.48(14) \\ \text { C191-C19-C19A-C114 } & 168.09(11) \\ \text { C114-C19A-C110-C111 } & -1.25(18) \\ \text { C19-C19A-C110-C111 } & -178.23(11) \\ \text { C19A-C110-C111-C112 } & 0.21(19) \\ \text { C19A-C110-C111-C116 } & -179.52(12) \\ \text { C110-C111-C112-C113 } & 1.25(19) \\ \text { C116-C111-C112-C113 } & -179.02(13) \\ \text { C111-C112-C113-C114 } & -1.7(2) \\ \text { C112-C113-C114-C19A } & 0.62(19) \\ \text { C112-C113-C114-C115 } & -176.88(12) \\ \text { C110-C19A-C114-C113 } & 0.82(18) \\ \text { C19-C19A-C114-C113 } & 177.80(11) \\ \text { C110-C19A-C114-C115 } & 178.04(11) \\ \text { C19-C19A-C114-C115 } & -4.98(19) \\ \text { C13-N14-C115-C114 } & -150.37(12) \\ \text { C14A-N14-C115-C114 } & 36.66(18) \\ \text { C13-N14-C115-S11 } & -28.03(14) \\ \text { C14A-N14-C115-S11 } & 159.00(11) \\ \text { C113-C114-C115-N14 } & -158.47(12) \\ \text { C19A-C114-C115-N14 } & 24.20(18) \\ \text { C113-C114-C115-S11 } & 83.55(12) \\ \text { C19A-C114-C115-S11 } & -93.77(13) \\ \text { C12-S11-C115-N14 } & 35.54(9) \\ \text { C12-S11-C115-C114 } & 162.03(10) \\ \text { C18A-C19-C191-C192 } & 66.55(14) \\ \text { C19A-C19-C191-C192 } & -169.01(11) \\ & \end{array}$

\begin{tabular}{|c|c|}
\hline $\mathrm{C} 25-\mathrm{C} 26-\mathrm{C} 27-\mathrm{C} 28$ & $0.4(2)$ \\
\hline $\mathrm{C} 26-\mathrm{C} 27-\mathrm{C} 28-\mathrm{C} 28 \mathrm{~A}$ & $0.9(2)$ \\
\hline $\mathrm{C} 27-\mathrm{C} 28-\mathrm{C} 28 \mathrm{~A}-\mathrm{C} 24 \mathrm{~A}$ & $-1.56(19)$ \\
\hline $\mathrm{C} 27-\mathrm{C} 28-\mathrm{C} 28 \mathrm{~A}-\mathrm{C} 29$ & $178.65(12)$ \\
\hline $\mathrm{C} 25-\mathrm{C} 24 \mathrm{~A}-\mathrm{C} 28 \mathrm{~A}-\mathrm{C} 28$ & $1.00(19)$ \\
\hline $\mathrm{N} 24-\mathrm{C} 24 \mathrm{~A}-\mathrm{C} 28 \mathrm{~A}-\mathrm{C} 28$ & $178.58(11)$ \\
\hline $\mathrm{C} 25-\mathrm{C} 24 \mathrm{~A}-\mathrm{C} 28 \mathrm{~A}-\mathrm{C} 29$ & $-179.20(12)$ \\
\hline $\mathrm{N} 24-\mathrm{C} 24 \mathrm{~A}-\mathrm{C} 28 \mathrm{~A}-\mathrm{C} 29$ & $-1.62(17)$ \\
\hline $\mathrm{C} 28-\mathrm{C} 28 \mathrm{~A}-\mathrm{C} 29-\mathrm{C} 29 \mathrm{~A}$ & $106.61(13)$ \\
\hline $\mathrm{C} 24 \mathrm{~A}-\mathrm{C} 28 \mathrm{~A}-\mathrm{C} 29-\mathrm{C} 29 \mathrm{~A}$ & $-73.18(13)$ \\
\hline $\mathrm{C} 28-\mathrm{C} 28 \mathrm{~A}-\mathrm{C} 29-\mathrm{C} 291$ & $-23.55(17)$ \\
\hline $\mathrm{C} 24 \mathrm{~A}-\mathrm{C} 28 \mathrm{~A}-\mathrm{C} 29-\mathrm{C} 291$ & $156.66(11)$ \\
\hline $\mathrm{C} 28 \mathrm{~A}-\mathrm{C} 29-\mathrm{C} 29 \mathrm{~A}-\mathrm{C} 210$ & $-117.02(12)$ \\
\hline $\mathrm{C} 291-\mathrm{C} 29-\mathrm{C} 29 \mathrm{~A}-\mathrm{C} 210$ & $12.01(16)$ \\
\hline $\mathrm{C} 28 \mathrm{~A}-\mathrm{C} 29-\mathrm{C} 29 \mathrm{~A}-\mathrm{C} 214$ & $62.82(13)$ \\
\hline $\mathrm{C} 291-\mathrm{C} 29-\mathrm{C} 29 \mathrm{~A}-\mathrm{C} 214$ & $-168.16(10)$ \\
\hline $\mathrm{C} 214-\mathrm{C} 29 \mathrm{~A}-\mathrm{C} 210-\mathrm{C} 211$ & $1.41(17)$ \\
\hline $\mathrm{C} 29-\mathrm{C} 29 \mathrm{~A}-\mathrm{C} 210-\mathrm{C} 211$ & $-178.75(11)$ \\
\hline $\mathrm{C} 29 \mathrm{~A}-\mathrm{C} 210-\mathrm{C} 211-\mathrm{C} 212$ & $-2.46(18)$ \\
\hline $\mathrm{C} 29 \mathrm{~A}-\mathrm{C} 210-\mathrm{C} 211-\mathrm{C} 216$ & $176.74(11)$ \\
\hline $\mathrm{C} 210-\mathrm{C} 211-\mathrm{C} 212-\mathrm{C} 213$ & $1.27(18)$ \\
\hline $\mathrm{C} 216-\mathrm{C} 211-\mathrm{C} 212-\mathrm{C} 213$ & $-177.94(12)$ \\
\hline $\mathrm{C} 211-\mathrm{C} 212-\mathrm{C} 213-\mathrm{C} 214$ & $0.92(18)$ \\
\hline $\mathrm{C} 212-\mathrm{C} 213-\mathrm{C} 214-\mathrm{C} 29 \mathrm{~A}$ & $-1.98(18)$ \\
\hline $\mathrm{C} 212-\mathrm{C} 213-\mathrm{C} 214-\mathrm{C} 215$ & $174.33(11)$ \\
\hline $\mathrm{C} 210-\mathrm{C} 29 \mathrm{~A}-\mathrm{C} 214-\mathrm{C} 213$ & $0.81(16)$ \\
\hline $\mathrm{C} 29-\mathrm{C} 29 \mathrm{~A}-\mathrm{C} 214-\mathrm{C} 213$ & $-179.03(11)$ \\
\hline $\mathrm{C} 210-\mathrm{C} 29 \mathrm{~A}-\mathrm{C} 214-\mathrm{C} 215$ & $-175.05(11)$ \\
\hline $\mathrm{C} 29-\mathrm{C} 29 \mathrm{~A}-\mathrm{C} 214-\mathrm{C} 215$ & $5.11(17)$ \\
\hline $\mathrm{C} 23-\mathrm{N} 24-\mathrm{C} 215-\mathrm{C} 214$ & $146.00(12)$ \\
\hline $\mathrm{C} 24 \mathrm{~A}-\mathrm{N} 24-\mathrm{C} 215-\mathrm{C} 214$ & $-38.03(17)$ \\
\hline $\mathrm{C} 23-\mathrm{N} 24-\mathrm{C} 215-\mathrm{S} 21$ & $24.98(13)$ \\
\hline $\mathrm{C} 24 \mathrm{~A}-\mathrm{N} 24-\mathrm{C} 215-\mathrm{S} 21$ & $-159.05(10)$ \\
\hline $\mathrm{C} 213-\mathrm{C} 214-\mathrm{C} 215-\mathrm{N} 24$ & $161.04(10)$ \\
\hline $\mathrm{C} 29 \mathrm{~A}-\mathrm{C} 214-\mathrm{C} 215-\mathrm{N} 24$ & $-22.92(17)$ \\
\hline $\mathrm{C} 213-\mathrm{C} 214-\mathrm{C} 215-\mathrm{S} 21$ & $-81.06(12)$ \\
\hline $\mathrm{C} 29 \mathrm{~A}-\mathrm{C} 214-\mathrm{C} 215-\mathrm{S} 21$ & $94.98(12)$ \\
\hline $\mathrm{C} 22-\mathrm{S} 21-\mathrm{C} 215-\mathrm{N} 24$ & $-30.96(9)$ \\
\hline $\mathrm{C} 22-\mathrm{S} 21-\mathrm{C} 215-\mathrm{C} 214$ & $-157.65(10)$ \\
\hline $\mathrm{C} 29 \mathrm{~A}-\mathrm{C} 29-\mathrm{C} 291-\mathrm{C} 292$ & $170.60(10)$ \\
\hline $\mathrm{C} 28 \mathrm{~A}-\mathrm{C} 29-\mathrm{C} 291-\mathrm{C} 292$ & $-62.94(13)$ \\
\hline
\end{tabular}

Hydrogen-bond geometry $\left(\AA,{ }^{\circ}\right)$

\begin{tabular}{lllll}
\hline$D-\mathrm{H} \cdots A$ & $D-\mathrm{H}$ & $\mathrm{H} \cdots A$ & $D \cdots A$ & $D-\mathrm{H} \cdots A$ \\
\hline $\mathrm{C} 115-\mathrm{H} 115 \cdots \mathrm{O} 13^{\mathrm{i}}$ & 1.00 & 2.47 & $3.3084(18)$ & 141 \\
$\mathrm{C} 215-\mathrm{H} 215 \cdots \mathrm{O} 23^{\mathrm{ii}}$ & 1.00 & 2.37 & $3.1681(18)$ & 136
\end{tabular}




\begin{tabular}{lllll}
$\mathrm{C} 112-\mathrm{H} 112 \cdots C g 1^{1 \mathrm{iii}}$ & 0.95 & 2.81 & $3.6738(13)$ & 152 \\
$\mathrm{C} 113-\mathrm{H} 113 \cdots C g 2^{2 \mathrm{ii}}$ & 0.95 & 2.85 & $3.7676(14)$ & 163 \\
\hline
\end{tabular}

Symmetry codes: (i) $-x, y+1 / 2,-z+1$; (ii) $-x, y-1 / 2,-z$; (iii) $x, y+1, z$.

(II) $(9 R S, 15 R S)-9$-Ethyl-7,12-dimethyl-9,13b-dihydrodibenzo[c,f]thiazolo[3,2-a]azepin-3(2H)-one

\section{Crystal data}

$\mathrm{C}_{20} \mathrm{H}_{21} \mathrm{NOS}$

$M_{r}=323.44$

Monoclinic, $C 2 / c$

$a=18.1021(12) \AA$

$b=12.4436(8) \AA$

$c=14.8429$ (9) $\AA$

$\beta=97.645(3)^{\circ}$

$V=3313.7(4) \AA^{3}$

$Z=8$

\section{Data collection}

Bruker Kappa APEXII diffractometer

Radiation source: high brilliance microfocus sealed tube

$\varphi$ and $\omega$ scans

Absorption correction: multi-scan

(SADABS; Bruker, 2006)

$T_{\text {min }}=0.818, T_{\text {max }}=0.961$

Refinement

Refinement on $F^{2}$

Least-squares matrix: full

$R\left[F^{2}>2 \sigma\left(F^{2}\right)\right]=0.060$

$w R\left(F^{2}\right)=0.168$

$S=1.05$

3402 reflections

211 parameters

0 restraints
$F(000)=1376$

$D_{\mathrm{x}}=1.297 \mathrm{Mg} \mathrm{m}^{-3}$

Mo $K \alpha$ radiation, $\lambda=0.71073 \AA$

Cell parameters from 3402 reflections

$\theta=2.0-26.4^{\circ}$

$\mu=0.20 \mathrm{~mm}^{-1}$

$T=298 \mathrm{~K}$

Block, colourless

$0.23 \times 0.22 \times 0.20 \mathrm{~mm}$

Special details

Geometry. All esds (except the esd in the dihedral angle between two 1.s. planes) are estimated using the full covariance matrix. The cell esds are taken into account individually in the estimation of esds in distances, angles and torsion angles; correlations between esds in cell parameters are only used when they are defined by crystal symmetry. An approximate (isotropic) treatment of cell esds is used for estimating esds involving l.s. planes.

Fractional atomic coordinates and isotropic or equivalent isotropic displacement parameters $\left(\AA^{2}\right)$

\begin{tabular}{lllll}
\hline & $x$ & $y$ & $z$ & $U_{\text {iso }} * / U_{\text {eq }}$ \\
\hline S1 & $0.40943(5)$ & $0.29645(9)$ & $0.65893(6)$ & $0.0801(4)$ \\
C2 & $0.32187(17)$ & $0.2270(3)$ & $0.6390(2)$ & $0.0610(8)$ \\
H2A & 0.3186 & 0.1744 & 0.6866 & $0.073^{*}$ \\
H2B & 0.2811 & 0.2776 & 0.6391 & $0.073^{*}$ \\
C3 & $0.31728(16)$ & $0.1719(2)$ & $0.5480(2)$ & $0.0524(7)$ \\
O3 & $0.26206(12)$ & $0.12615(19)$ & $0.51154(16)$ & $0.0669(6)$
\end{tabular}




$\begin{array}{lllll}\text { N4 } & 0.38267(12) & 0.18081(18) & 0.51161(15) & 0.0466(6) \\ \text { C4A } & 0.38797(15) & 0.1538(2) & 0.41955(18) & 0.0453(6) \\ \text { C5 } & 0.37768(18) & 0.0494(2) & 0.3880(2) & 0.0589(8) \\ \text { H5 } & 0.3673 & -0.0052 & 0.4272 & 0.071^{*} \\ \text { C6 } & 0.38296(18) & 0.0273(2) & 0.2984(2) & 0.0612(8) \\ \text { H6 } & 0.3754 & -0.0429 & 0.2775 & 0.073^{*} \\ \text { C7 } & 0.39919(15) & 0.1061(2) & 0.23845(19) & 0.0488(7) \\ \text { C8 } & 0.40901(14) & 0.2104(2) & 0.27151(17) & 0.0421(6) \\ \text { H8 } & 0.4194 & 0.2648 & 0.2320 & 0.051^{*} \\ \text { C8A } & 0.40380(13) & 0.23624(19) & 0.36146(17) & 0.0392^{(6)} \\ \text { C9 } & 0.41873(14) & 0.34878(19) & 0.40082(17) & 0.0403(6) \\ \text { H9 } & 0.3821 & 0.3619 & 0.4427 & 0.048^{*} \\ \text { C9A } & 0.49525(14) & 0.34356(19) & 0.45798(17) & 0.0405(6) \\ \text { C10 } & 0.55658(16) & 0.4003(2) & 0.43479(19) & 0.0486(7) \\ \text { H170 } & 0.5497 & 0.4480 & 0.3862 & 0.058^{*} \\ \text { C11 } & 0.62705(16) & 0.3875(3) & 0.4820(2) & 0.0571(8) \\ \text { H11 } & 0.6664 & 0.4268 & 0.4645 & 0.069^{*} \\ \text { C12 } & 0.64037(16) & 0.3175(3) & 0.5547(2) & 0.0548(8) \\ \text { C13 } & 0.57916(15) & 0.2630(2) & 0.57933(19) & 0.0494(7) \\ \text { H13 } & 0.5863 & 0.2162 & 0.6286 & 0.059^{*} \\ \text { C14 } & 0.50769(14) & 0.2758(2) & 0.53333(17) & 0.0425(6) \\ \text { C15 } & 0.44750(15) & 0.2154(2) & 0.57304(18) & 0.0484(7) \\ \text { H15 } & 0.4703 & 0.1512 & 0.6034 & 0.058^{*} \\ \text { C71 } & 0.40746(18) & 0.0815(3) & 0.1414(2) & 0.0613(8) \\ \text { H71A } & 0.3721 & 0.0272 & 0.1187 & 0.092^{*} \\ \text { H71B } & 0.4571 & 0.0560 & 0.1379 & 0.092^{*} \\ \text { H71C } & 0.3985 & 0.1455 & 0.1055 & 0.092^{*} \\ \text { C91 } & 0.40972(15) & 0.4383(2) & 0.33031(18) & 0.0475(7) \\ \text { H91A } & 0.4249 & 0.5058 & 0.3598 & 0.057^{*} \\ \text { H91B } & 0.4426 & 0.4243 & 0.2851 & 0.057^{*} \\ \text { C92 } & 0.33049(18) & 0.4491(3) & 0.2831(2) & 0.0630(8) \\ \text { H92A } & 0.3276 & 0.5085 & 0.2415 & 0.095^{*} \\ \text { H92B } & 0.2975 & 0.4614 & 0.3276 & 0.095^{*} \\ \text { H92C } & 0.3162 & 0.3842 & 0.2503 & 0.095^{*} \\ \text { C121 } & 0.71688(18) & 0.3000(3) & 0.6059(3) & 0.0804(11) \\ \text { H12A } & 0.7308 & 0.2260 & 0.6009 & 0.121^{*} \\ \text { H12B } & 0.7166 & 0.3179 & 0.6687 & 0.121^{*} \\ \text { H12C } & 0.7521 & 0.3449 & 0.5807 & 0.121^{*} \\ & & & & \end{array}$

Atomic displacement parameters $\left(\AA^{2}\right)$

\begin{tabular}{lllllll}
\hline & $U^{11}$ & $U^{22}$ & $U^{33}$ & $U^{12}$ & $U^{13}$ & $U^{23}$ \\
\hline S1 & $0.0711(6)$ & $0.1163(8)$ & $0.0586(5)$ & $-0.0213(5)$ & $0.0302(4)$ & $-0.0193(5)$ \\
C2 & $0.0511(17)$ & $0.081(2)$ & $0.0540(17)$ & $0.0063(16)$ & $0.0195(14)$ & $0.0199(16)$ \\
C3 & $0.0453(16)$ & $0.0565(17)$ & $0.0574(17)$ & $0.0010(14)$ & $0.0140(13)$ & $0.0231(14)$ \\
O3 & $0.0440(12)$ & $0.0749(15)$ & $0.0830(15)$ & $-0.0105(11)$ & $0.0124(11)$ & $0.0168(12)$ \\
N4 & $0.0427(13)$ & $0.0499(13)$ & $0.0489(13)$ & $-0.0057(10)$ & $0.0120(10)$ & $0.0065(10)$ \\
C4A & $0.0408(14)$ & $0.0438(14)$ & $0.0523(15)$ & $-0.0048(11)$ & $0.0094(12)$ & $0.0030(12)$
\end{tabular}


supporting information

$\begin{array}{lllllll}\text { C5 } & 0.067(2) & 0.0446(16) & 0.0644(19) & -0.0152(14) & 0.0072(15) & 0.0090(14) \\ \text { C6 } & 0.073(2) & 0.0397(15) & 0.068(2) & -0.0096(14) & -0.0006(16) & -0.0059(14) \\ \text { C7 } & 0.0444(15) & 0.0468(15) & 0.0538(16) & 0.0003(12) & 0.0012(12) & -0.0047(13) \\ \text { C8 } & 0.0377(14) & 0.0407(14) & 0.0488(15) & -0.0003(11) & 0.0090(11) & 0.0026(12) \\ \text { C8A } & 0.0317(12) & 0.0362(13) & 0.0509(15) & -0.0005(10) & 0.0099(11) & 0.0021(11) \\ \text { C9 } & 0.0430(14) & 0.0376(13) & 0.0441(14) & 0.0001(11) & 0.0192(11) & -0.0010(11) \\ \text { C9A } & 0.0410(14) & 0.0358(13) & 0.0474(15) & -0.0020(11) & 0.0159(11) & -0.0077(11) \\ \text { C10 } & 0.0544(17) & 0.0455(15) & 0.0497(15) & -0.0087(13) & 0.0211(13) & -0.0082(12) \\ \text { C11 } & 0.0441(17) & 0.0680(19) & 0.0632(19) & -0.0156(14) & 0.0218(14) & -0.0191(16) \\ \text { C12 } & 0.0428(16) & 0.0672(19) & 0.0559(17) & -0.0022(13) & 0.0125(13) & -0.0187(15) \\ \text { C13 } & 0.0475(16) & 0.0523(16) & 0.0488(15) & 0.0031(13) & 0.0084(13) & -0.0083(13) \\ \text { C14 } & 0.0425(15) & 0.0416(14) & 0.0456(14) & -0.0004(11) & 0.0145(12) & -0.0058(11) \\ \text { C15 } & 0.0471(16) & 0.0520(16) & 0.0470(15) & -0.0037(13) & 0.0097(12) & 0.0064(13) \\ \text { C71 } & 0.064(2) & 0.0570(18) & 0.0623(19) & 0.0020(15) & 0.0050(15) & -0.0142(15) \\ \text { C91 } & 0.0530(16) & 0.0403(14) & 0.0530(16) & 0.0013(12) & 0.0208(13) & 0.0024(12) \\ \text { C92 } & 0.063(2) & 0.0562(18) & 0.071(2) & 0.0113(15) & 0.0147(16) & 0.0179(15) \\ \text { C121 } & 0.0466(19) & 0.111(3) & 0.082(2) & -0.0024(19) & 0.0051(17) & -0.022(2) \\ & & & & & & \end{array}$

Geometric parameters (A, $\stackrel{\circ}{)}$

\begin{tabular}{llll}
\hline $\mathrm{S} 1-\mathrm{C} 2$ & $1.795(3)$ & $\mathrm{C} 9 \mathrm{~A}-\mathrm{C} 10$ & $1.397(4)$ \\
$\mathrm{S} 1-\mathrm{C} 15$ & $1.831(3)$ & $\mathrm{C} 10-\mathrm{C} 11$ & $1.381(4)$ \\
$\mathrm{C} 2-\mathrm{C} 3$ & $1.508(4)$ & $\mathrm{C} 10-\mathrm{H} 170$ & 0.9300 \\
$\mathrm{C} 2-\mathrm{H} 2 \mathrm{~A}$ & 0.9700 & $\mathrm{C} 11-\mathrm{C} 12$ & $1.382(4)$ \\
$\mathrm{C} 2-\mathrm{H} 2 \mathrm{~B}$ & 0.9700 & $\mathrm{C} 11-\mathrm{H} 11$ & 0.9300 \\
$\mathrm{C} 3-\mathrm{O} 3$ & $1.214(4)$ & $\mathrm{C} 12-\mathrm{C} 13$ & $1.389(4)$ \\
$\mathrm{C} 3-\mathrm{N} 4$ & $1.369(3)$ & $\mathrm{C} 12-\mathrm{C} 121$ & $1.504(4)$ \\
$\mathrm{N} 4-\mathrm{C} 4 \mathrm{~A}$ & $1.423(3)$ & $\mathrm{C} 13-\mathrm{C} 14$ & $1.389(4)$ \\
$\mathrm{N} 4-\mathrm{C} 15$ & $1.452(3)$ & $\mathrm{C} 13-\mathrm{H} 13$ & 0.9300 \\
$\mathrm{C} 4 \mathrm{~A}-\mathrm{C} 5$ & $1.385(4)$ & $\mathrm{C} 14-\mathrm{C} 15$ & $1.507(4)$ \\
$\mathrm{C} 4 \mathrm{~A}-\mathrm{C} 8 \mathrm{~A}$ & $1.394(3)$ & $\mathrm{C} 15-\mathrm{H} 15$ & 0.9800 \\
$\mathrm{C} 5-\mathrm{C} 6$ & $1.374(4)$ & $\mathrm{C} 71-\mathrm{H} 71 \mathrm{~A}$ & 0.9600 \\
$\mathrm{C} 5-\mathrm{H} 5$ & 0.9300 & $\mathrm{C} 71-\mathrm{H} 71 \mathrm{~B}$ & 0.9600 \\
$\mathrm{C} 6-\mathrm{C} 7$ & $1.382(4)$ & $\mathrm{C} 71-\mathrm{H} 71 \mathrm{C}$ & 0.9600 \\
$\mathrm{C} 6-\mathrm{H} 6$ & 0.9300 & $\mathrm{C} 91-\mathrm{C} 92$ & $1.516(4)$ \\
$\mathrm{C} 7-\mathrm{C} 8$ & $1.391(4)$ & $\mathrm{C} 91-\mathrm{H} 91 \mathrm{~A}$ & 0.9700 \\
$\mathrm{C} 7-\mathrm{C} 71$ & $1.499(4)$ & $\mathrm{C} 91-\mathrm{H} 91 \mathrm{~B}$ & 0.9700 \\
$\mathrm{C} 8-\mathrm{C} 8 \mathrm{~A}$ & $1.389(3)$ & $\mathrm{C} 92-\mathrm{H} 92 \mathrm{~A}$ & 0.9600 \\
$\mathrm{C} 8-\mathrm{H} 8$ & 0.9300 & $\mathrm{C} 92-\mathrm{H} 92 \mathrm{~B}$ & 0.9600 \\
$\mathrm{C} 8 \mathrm{~A}-\mathrm{C} 9$ & $1.528(3)$ & $\mathrm{C} 92-\mathrm{H} 92 \mathrm{C}$ & 0.9600 \\
$\mathrm{C} 9-\mathrm{C} 91$ & $1.522(3)$ & $\mathrm{C} 121-\mathrm{H} 12 \mathrm{~A}$ & 0.9600 \\
$\mathrm{C} 9-\mathrm{C} 9 \mathrm{~A}$ & $1.527(4)$ & $\mathrm{C} 121-\mathrm{H} 12 \mathrm{~B}$ & 0.9600 \\
$\mathrm{C} 9-\mathrm{H} 9$ & 0.9800 & $\mathrm{C} 121-\mathrm{H} 12 \mathrm{C}$ & 0.9600 \\
$\mathrm{C} 9 \mathrm{~A}-\mathrm{C} 14$ & $1.395(4)$ & & 119.3 \\
$\mathrm{C} 2-\mathrm{S} 1-\mathrm{C} 15$ & & $\mathrm{C} 10-\mathrm{C} 11-\mathrm{C} 12$ & 119.3 \\
$\mathrm{C} 3-\mathrm{C} 2-\mathrm{S} 1$ & $91.50(14)$ & $\mathrm{C} 10-\mathrm{C} 11-\mathrm{H} 11$ & \\
$\mathrm{C} 3-\mathrm{C} 2-\mathrm{H} 2 \mathrm{~A}$ & $107.97(19)$ & $\mathrm{C} 12-\mathrm{C} 11-\mathrm{H} 11$ & \\
& 110.1 & &
\end{tabular}




\begin{tabular}{|c|c|c|c|}
\hline $\mathrm{S} 1-\mathrm{C} 2-\mathrm{H} 2 \mathrm{~A}$ & 110.1 & $\mathrm{C} 11-\mathrm{C} 12-\mathrm{C} 13$ & $116.8(3)$ \\
\hline $\mathrm{C} 3-\mathrm{C} 2-\mathrm{H} 2 \mathrm{~B}$ & 110.1 & $\mathrm{C} 11-\mathrm{C} 12-\mathrm{C} 121$ & $122.6(3)$ \\
\hline $\mathrm{S} 1-\mathrm{C} 2-\mathrm{H} 2 \mathrm{~B}$ & 110.1 & $\mathrm{C} 13-\mathrm{C} 12-\mathrm{C} 121$ & $120.6(3)$ \\
\hline $\mathrm{H} 2 \mathrm{~A}-\mathrm{C} 2-\mathrm{H} 2 \mathrm{~B}$ & 108.4 & $\mathrm{C} 12-\mathrm{C} 13-\mathrm{C} 14$ & $122.6(3)$ \\
\hline $\mathrm{O} 3-\mathrm{C} 3-\mathrm{N} 4$ & $124.5(3)$ & $\mathrm{C} 12-\mathrm{C} 13-\mathrm{H} 13$ & 118.7 \\
\hline $\mathrm{O} 3-\mathrm{C} 3-\mathrm{C} 2$ & $123.8(3)$ & $\mathrm{C} 14-\mathrm{C} 13-\mathrm{H} 13$ & 118.7 \\
\hline $\mathrm{N} 4-\mathrm{C} 3-\mathrm{C} 2$ & $111.7(3)$ & $\mathrm{C} 13-\mathrm{C} 14-\mathrm{C} 9 \mathrm{~A}$ & $120.2(2)$ \\
\hline $\mathrm{C} 3-\mathrm{N} 4-\mathrm{C} 4 \mathrm{~A}$ & $122.1(2)$ & $\mathrm{C} 13-\mathrm{C} 14-\mathrm{C} 15$ & $115.2(2)$ \\
\hline $\mathrm{C} 3-\mathrm{N} 4-\mathrm{C} 15$ & $116.4(2)$ & C9A-C14-C15 & $124.6(2)$ \\
\hline $\mathrm{C} 4 \mathrm{~A}-\mathrm{N} 4-\mathrm{C} 15$ & $121.4(2)$ & $\mathrm{N} 4-\mathrm{C} 15-\mathrm{C} 14$ & $117.9(2)$ \\
\hline $\mathrm{C} 5-\mathrm{C} 4 \mathrm{~A}-\mathrm{C} 8 \mathrm{~A}$ & $120.7(3)$ & $\mathrm{N} 4-\mathrm{C} 15-\mathrm{S} 1$ & $104.64(18)$ \\
\hline $\mathrm{C} 5-\mathrm{C} 4 \mathrm{~A}-\mathrm{N} 4$ & $121.5(2)$ & $\mathrm{C} 14-\mathrm{C} 15-\mathrm{S} 1$ & $110.88(19)$ \\
\hline $\mathrm{C} 8 \mathrm{~A}-\mathrm{C} 4 \mathrm{~A}-\mathrm{N} 4$ & $117.8(2)$ & $\mathrm{N} 4-\mathrm{C} 15-\mathrm{H} 15$ & 107.7 \\
\hline $\mathrm{C} 6-\mathrm{C} 5-\mathrm{C} 4 \mathrm{~A}$ & $119.5(3)$ & $\mathrm{C} 14-\mathrm{C} 15-\mathrm{H} 15$ & 107.7 \\
\hline $\mathrm{C} 6-\mathrm{C} 5-\mathrm{H} 5$ & 120.3 & $\mathrm{~S} 1-\mathrm{C} 15-\mathrm{H} 15$ & 107.7 \\
\hline $\mathrm{C} 4 \mathrm{~A}-\mathrm{C} 5-\mathrm{H} 5$ & 120.3 & C7-C71-H71A & 109.5 \\
\hline $\mathrm{C} 5-\mathrm{C} 6-\mathrm{C} 7$ & $121.9(3)$ & C7-C71-H71B & 109.5 \\
\hline $\mathrm{C} 5-\mathrm{C} 6-\mathrm{H} 6$ & 119.0 & $\mathrm{H} 71 \mathrm{~A}-\mathrm{C} 71-\mathrm{H} 71 \mathrm{~B}$ & 109.5 \\
\hline $\mathrm{C} 7-\mathrm{C} 6-\mathrm{H} 6$ & 119.0 & $\mathrm{C} 7-\mathrm{C} 71-\mathrm{H} 71 \mathrm{C}$ & 109.5 \\
\hline $\mathrm{C} 6-\mathrm{C} 7-\mathrm{C} 8$ & $117.6(3)$ & $\mathrm{H} 71 \mathrm{~A}-\mathrm{C} 71-\mathrm{H} 71 \mathrm{C}$ & 109.5 \\
\hline $\mathrm{C} 6-\mathrm{C} 7-\mathrm{C} 71$ & $122.0(3)$ & $\mathrm{H} 71 \mathrm{~B}-\mathrm{C} 71-\mathrm{H} 71 \mathrm{C}$ & 109.5 \\
\hline $\mathrm{C} 8-\mathrm{C} 7-\mathrm{C} 71$ & $120.3(3)$ & $\mathrm{C} 92-\mathrm{C} 91-\mathrm{C} 9$ & $113.0(2)$ \\
\hline $\mathrm{C} 8 \mathrm{~A}-\mathrm{C} 8-\mathrm{C} 7$ & $122.2(2)$ & C92-C91-H91A & 109.0 \\
\hline $\mathrm{C} 8 \mathrm{~A}-\mathrm{C} 8-\mathrm{H} 8$ & 118.9 & C9-C91-H91A & 109.0 \\
\hline $\mathrm{C} 7-\mathrm{C} 8-\mathrm{H} 8$ & 118.9 & C92-C91-H91B & 109.0 \\
\hline $\mathrm{C} 8-\mathrm{C} 8 \mathrm{~A}-\mathrm{C} 4 \mathrm{~A}$ & $118.1(2)$ & C9- $991-\mathrm{H} 91 \mathrm{~B}$ & 109.0 \\
\hline $\mathrm{C} 8-\mathrm{C} 8 \mathrm{~A}-\mathrm{C} 9$ & $123.3(2)$ & H91A-C91-H91B & 107.8 \\
\hline $\mathrm{C} 4 \mathrm{~A}-\mathrm{C} 8 \mathrm{~A}-\mathrm{C} 9$ & $118.6(2)$ & C91-C92-H92A & 109.5 \\
\hline $\mathrm{C} 91-\mathrm{C} 9-\mathrm{C} 9 \mathrm{~A}$ & $114.8(2)$ & C91-C92-H92B & 109.5 \\
\hline $\mathrm{C} 91-\mathrm{C} 9-\mathrm{C} 8 \mathrm{~A}$ & $114.2(2)$ & $\mathrm{H} 92 \mathrm{~A}-\mathrm{C} 92-\mathrm{H} 92 \mathrm{~B}$ & 109.5 \\
\hline $\mathrm{C} 9 \mathrm{~A}-\mathrm{C} 9-\mathrm{C} 8 \mathrm{~A}$ & $106.0(2)$ & $\mathrm{C} 91-\mathrm{C} 92-\mathrm{H} 92 \mathrm{C}$ & 109.5 \\
\hline C91-C9-H9 & 107.1 & $\mathrm{H} 92 \mathrm{~A}-\mathrm{C} 92-\mathrm{H} 92 \mathrm{C}$ & 109.5 \\
\hline $\mathrm{C} 9 \mathrm{~A}-\mathrm{C} 9-\mathrm{H} 9$ & 107.1 & $\mathrm{H} 92 \mathrm{~B}-\mathrm{C} 92-\mathrm{H} 92 \mathrm{C}$ & 109.5 \\
\hline $\mathrm{C} 8 \mathrm{~A}-\mathrm{C} 9-\mathrm{H} 9$ & 107.1 & $\mathrm{C} 12-\mathrm{C} 121-\mathrm{H} 12 \mathrm{~A}$ & 109.5 \\
\hline $\mathrm{C} 14-\mathrm{C} 9 \mathrm{~A}-\mathrm{C} 10$ & $117.0(2)$ & $\mathrm{C} 12-\mathrm{C} 121-\mathrm{H} 12 \mathrm{~B}$ & 109.5 \\
\hline $\mathrm{C} 14-\mathrm{C} 9 \mathrm{~A}-\mathrm{C} 9$ & $120.6(2)$ & $\mathrm{H} 12 \mathrm{~A}-\mathrm{C} 121-\mathrm{H} 12 \mathrm{~B}$ & 109.5 \\
\hline $\mathrm{C} 10-\mathrm{C} 9 \mathrm{~A}-\mathrm{C} 9$ & $122.3(2)$ & $\mathrm{C} 12-\mathrm{C} 121-\mathrm{H} 12 \mathrm{C}$ & 109.5 \\
\hline $\mathrm{C} 11-\mathrm{C} 10-\mathrm{C} 9 \mathrm{~A}$ & $121.9(3)$ & $\mathrm{H} 12 \mathrm{~A}-\mathrm{C} 121-\mathrm{H} 12 \mathrm{C}$ & 109.5 \\
\hline $\mathrm{C} 11-\mathrm{C} 10-\mathrm{H} 170$ & 119.1 & $\mathrm{H} 12 \mathrm{~B}-\mathrm{C} 121-\mathrm{H} 12 \mathrm{C}$ & 109.5 \\
\hline $\mathrm{C} 9 \mathrm{~A}-\mathrm{C} 10-\mathrm{H} 170$ & 119.1 & & \\
\hline $\mathrm{C} 15-\mathrm{S} 1-\mathrm{C} 2-\mathrm{C} 3$ & $-18.5(2)$ & $\mathrm{C} 8 \mathrm{~A}-\mathrm{C} 9-\mathrm{C} 9 \mathrm{~A}-\mathrm{C} 14$ & $-62.2(3)$ \\
\hline $\mathrm{S} 1-\mathrm{C} 2-\mathrm{C} 3-\mathrm{O} 3$ & $-173.3(2)$ & $\mathrm{C} 91-\mathrm{C} 9-\mathrm{C} 9 \mathrm{~A}-\mathrm{C} 10$ & $-13.3(3)$ \\
\hline $\mathrm{S} 1-\mathrm{C} 2-\mathrm{C} 3-\mathrm{N} 4$ & $6.3(3)$ & $\mathrm{C} 8 \mathrm{~A}-\mathrm{C} 9-\mathrm{C} 9 \mathrm{~A}-\mathrm{C} 10$ & $113.8(2)$ \\
\hline $\mathrm{O} 3-\mathrm{C} 3-\mathrm{N} 4-\mathrm{C} 4 \mathrm{~A}$ & $11.8(4)$ & $\mathrm{C} 14-\mathrm{C} 9 \mathrm{~A}-\mathrm{C} 10-\mathrm{C} 11$ & $2.2(4)$ \\
\hline $\mathrm{C} 2-\mathrm{C} 3-\mathrm{N} 4-\mathrm{C} 4 \mathrm{~A}$ & $-167.8(2)$ & $\mathrm{C} 9-\mathrm{C} 9 \mathrm{~A}-\mathrm{C} 10-\mathrm{C} 11$ & $-173.9(2)$ \\
\hline $\mathrm{O} 3-\mathrm{C} 3-\mathrm{N} 4-\mathrm{C} 15$ & $-165.9(3)$ & $\mathrm{C} 9 \mathrm{~A}-\mathrm{C} 10-\mathrm{C} 11-\mathrm{C} 12$ & $0.1(4)$ \\
\hline $\mathrm{C} 2-\mathrm{C} 3-\mathrm{N} 4-\mathrm{C} 15$ & $14.6(3)$ & $\mathrm{C} 10-\mathrm{C} 11-\mathrm{C} 12-\mathrm{C} 13$ & $-1.7(4)$ \\
\hline
\end{tabular}




$\begin{array}{llll}\mathrm{C} 3-\mathrm{N} 4-\mathrm{C} 4 \mathrm{~A}-\mathrm{C} 5 & -65.0(4) & \mathrm{C} 10-\mathrm{C} 11-\mathrm{C} 12-\mathrm{C} 121 & 178.5(3) \\ \mathrm{C} 15-\mathrm{N} 4-\mathrm{C} 4 \mathrm{~A}-\mathrm{C} 5 & 112.5(3) & \mathrm{C} 11-\mathrm{C} 12-\mathrm{C} 13-\mathrm{C} 14 & 0.9(4) \\ \mathrm{C} 3-\mathrm{N} 4-\mathrm{C} 4 \mathrm{~A}-\mathrm{C} 8 \mathrm{~A} & 114.9(3) & \mathrm{C} 121-\mathrm{C} 12-\mathrm{C} 13-\mathrm{C} 14 & -179.2(3) \\ \mathrm{C} 15-\mathrm{N} 4-\mathrm{C} 4 \mathrm{~A}-\mathrm{C} 8 \mathrm{~A} & -67.6(3) & \mathrm{C} 12-\mathrm{C} 13-\mathrm{C} 14-\mathrm{C} 9 \mathrm{~A} & 1.4(4) \\ \mathrm{C} 8 \mathrm{~A}-\mathrm{C} 4 \mathrm{~A}-\mathrm{C} 5-\mathrm{C} 6 & -0.2(4) & \mathrm{C} 12-\mathrm{C} 13-\mathrm{C} 14-\mathrm{C} 15 & -176.5(2) \\ \mathrm{N} 4-\mathrm{C} 4 \mathrm{~A}-\mathrm{C} 5-\mathrm{C} 6 & 179.7(3) & \mathrm{C} 10-\mathrm{C} 9 \mathrm{~A}-\mathrm{C} 14-\mathrm{C} 13 & -2.9(3) \\ \mathrm{C} 4 \mathrm{~A}-\mathrm{C} 5-\mathrm{C} 6-\mathrm{C} 7 & 0.8(5) & \mathrm{C} 9-\mathrm{C} 9 \mathrm{~A}-\mathrm{C} 14-\mathrm{C} 13 & 173.3(2) \\ \mathrm{C} 5-\mathrm{C} 6-\mathrm{C} 7-\mathrm{C} 8 & -1.0(4) & \mathrm{C} 10-\mathrm{C} 9 \mathrm{~A}-\mathrm{C} 14-\mathrm{C} 15 & 174.8(2) \\ \mathrm{C} 5-\mathrm{C} 6-\mathrm{C} 7-\mathrm{C} 71 & 178.0(3) & \mathrm{C} 9-\mathrm{C} 9 \mathrm{~A}-\mathrm{C} 14-\mathrm{C} 15 & -9.0(4) \\ \mathrm{C} 6-\mathrm{C} 7-\mathrm{C} 8-\mathrm{C} 8 \mathrm{~A} & 0.7(4) & \mathrm{C} 3-\mathrm{N} 4-\mathrm{C} 15-\mathrm{C} 14 & -151.3(2) \\ \mathrm{C} 71-\mathrm{C} 7-\mathrm{C} 8-\mathrm{C} 8 \mathrm{~A} & -178.3(3) & \mathrm{C} 4 \mathrm{~A}-\mathrm{N} 4-\mathrm{C} 15-\mathrm{C} 14 & 31.0(4) \\ \mathrm{C} 7-\mathrm{C} 8-\mathrm{C} 8 \mathrm{~A}-\mathrm{C} 4 \mathrm{~A} & -0.2(4) & \mathrm{C} 3-\mathrm{N} 4-\mathrm{C} 15-\mathrm{S} 1 & -27.6(3) \\ \mathrm{C} 7-\mathrm{C} 8-\mathrm{C} 8 \mathrm{~A}-\mathrm{C} 9 & 176.4(2) & \mathrm{C} 4 \mathrm{~A}-\mathrm{N} 4-\mathrm{C} 15-\mathrm{S} 1 & 154.7(2) \\ \mathrm{C} 5-\mathrm{C} 4 \mathrm{~A}-\mathrm{C} 8 \mathrm{~A}-\mathrm{C} 8 & -0.1(4) & \mathrm{C} 13-\mathrm{C} 14-\mathrm{C} 15-\mathrm{N} 4 & -150.7(2) \\ \mathrm{N} 4-\mathrm{C} 4 \mathrm{~A}-\mathrm{C} 8 \mathrm{~A}-\mathrm{C} 8 & -180.0(2) & \mathrm{C} 9 \mathrm{~A}-\mathrm{C} 14-\mathrm{C} 15-\mathrm{N} 4 & 31.5(4) \\ \mathrm{C} 5-\mathrm{C} 4 \mathrm{~A}-\mathrm{C} 8 \mathrm{~A}-\mathrm{C} 9 & -176.8(2) & \mathrm{C} 13-\mathrm{C} 14-\mathrm{C} 15-\mathrm{S} 1 & 88.7(3) \\ \mathrm{N} 4-\mathrm{C} 4 \mathrm{~A}-\mathrm{C} 8 \mathrm{~A}-\mathrm{C} 9 & 3.3(4) & \mathrm{C} 9 \mathrm{~A}-\mathrm{C} 14-\mathrm{C} 15-\mathrm{S} 1 & -89.0(3) \\ \mathrm{C} 8-\mathrm{C} 8 \mathrm{~A}-\mathrm{C} 9-\mathrm{C} 91 & 22.9(3) & \mathrm{C} 2-\mathrm{S} 1-\mathrm{C} 15-\mathrm{N} 4 & 25.27(19) \\ \mathrm{C} 4 \mathrm{~A}-\mathrm{C} 8 \mathrm{~A}-\mathrm{C} 9-\mathrm{C} 91 & -160.6(2) & \mathrm{C} 2-\mathrm{S} 1-\mathrm{C} 15-\mathrm{C} 14 & 153.4(2) \\ \mathrm{C} 8-\mathrm{C} 8 \mathrm{~A}-\mathrm{C} 9-\mathrm{C} 9 \mathrm{~A} & -104.5(3) & \mathrm{C} 9 \mathrm{~A}-\mathrm{C} 9-\mathrm{C} 91-\mathrm{C} 92 & -173.0(2) \\ \mathrm{C} 4 \mathrm{~A}-\mathrm{C} 8 \mathrm{~A}-\mathrm{C} 9-\mathrm{C} 9 \mathrm{~A} & 72.0(3) & \mathrm{C} 8 \mathrm{~A}-\mathrm{C} 9-\mathrm{C} 91-\mathrm{C} 92 & 64.3(3) \\ \mathrm{C} 91-\mathrm{C} 9-\mathrm{C} 9 \mathrm{~A}-\mathrm{C} 14 & 170.8(2) & & \end{array}$

Hydrogen-bond geometry $\left(\AA,{ }^{\circ}\right)$

\begin{tabular}{lllll}
\hline$D-\mathrm{H} \cdots A$ & $D-\mathrm{H}$ & $\mathrm{H} \cdots A$ & $D \cdots A$ & $D-\mathrm{H} \cdots A$ \\
\hline $\mathrm{C} 2-\mathrm{H} 2 B \cdots \mathrm{O} 3^{\mathrm{i}}$ & 0.97 & 2.57 & $3.120(4)$ & 116 \\
$\mathrm{C} 91-\mathrm{H} 91 A \cdots C g 3^{\mathrm{ii}}$ & 0.97 & 2.82 & $3.736(3)$ & 157 \\
\hline
\end{tabular}

Symmetry codes: (i) $-x+1 / 2,-y+1 / 2,-z+1$; (ii) $-x+1,-y+1,-z+1$.

(III) Ethyl 2-chloro-13-ethyl-4-oxo-8,13-dihydro-4H-benzo[5,6]azepino[3,2,1-ij]quinoline-5-carboxylate

Crystal data

$\mathrm{C}_{22} \mathrm{H}_{20} \mathrm{ClNO}_{3}$

$M_{r}=381.84$

Triclinic, $P \overline{1}$

$a=6.8533(16) \AA$

$b=10.612(5) \AA$

$c=13.561(3) \AA$

$\alpha=72.45(4)^{\circ}$

$\beta=75.840(19)^{\circ}$

$\gamma=82.46(2)^{\circ}$

$V=910.0(6) \AA^{3}$

Data collection

Nonius KappaCCD

diffractometer

Radiation source: fine focus sealed tube Graphite monochromator $\varphi$ and $\omega$ scans
$Z=2$

$F(000)=400$

$D_{\mathrm{x}}=1.394 \mathrm{Mg} \mathrm{m}^{-3}$

Mo $K \alpha$ radiation, $\lambda=0.71073 \AA$

Cell parameters from 4176 reflections

$\theta=2.9-27.5^{\circ}$

$\mu=0.23 \mathrm{~mm}^{-1}$

$T=120 \mathrm{~K}$

Needle, colourless

$0.26 \times 0.15 \times 0.13 \mathrm{~mm}$

Absorption correction: multi-scan

(SADABS; Sheldrick, 2003)

$T_{\min }=0.845, T_{\max }=0.970$

20183 measured reflections

3776 independent reflections 
2195 reflections with $I>2 \sigma(I)$

$R_{\text {int }}=0.154$

$\theta_{\max }=26.6^{\circ}, \theta_{\min }=3.8^{\circ}$

\section{Refinement}

Refinement on $F^{2}$

Least-squares matrix: full

$R\left[F^{2}>2 \sigma\left(F^{2}\right)\right]=0.065$

$w R\left(F^{2}\right)=0.118$

$S=1.06$

3776 reflections

322 parameters

74 restraints

$$
\begin{aligned}
& h=-8 \rightarrow 8 \\
& k=-13 \rightarrow 13 \\
& l=-16 \rightarrow 17
\end{aligned}
$$

Hydrogen site location: inferred from neighbouring sites

$\mathrm{H}$-atom parameters constrained

$w=1 /\left[\sigma^{2}\left(F_{\mathrm{o}}^{2}\right)+(0.0192 P)^{2}+1.1423 P\right]$

where $P=\left(F_{\mathrm{o}}^{2}+2 F_{\mathrm{c}}^{2}\right) / 3$

$(\Delta / \sigma)_{\max }<0.001$

$\Delta \rho_{\max }=0.29 \mathrm{e} \AA^{-3}$

$\Delta \rho_{\min }=-0.31$ e $\AA^{-3}$

Special details

Geometry. All esds (except the esd in the dihedral angle between two 1.s. planes) are estimated using the full covariance matrix. The cell esds are taken into account individually in the estimation of esds in distances, angles and torsion angles; correlations between esds in cell parameters are only used when they are defined by crystal symmetry. An approximate (isotropic) treatment of cell esds is used for estimating esds involving l.s. planes.

Fractional atomic coordinates and isotropic or equivalent isotropic displacement parameters $\left(\AA^{2}\right)$

\begin{tabular}{llllll}
\hline & $x$ & $y$ & $z$ & $U_{\text {iso }} / U_{\text {eq }}$ & Occ. $(<1)$ \\
\hline C11 & $0.8032(8)$ & $0.3061(4)$ & $0.6534(3)$ & $0.0131(8)$ & $0.900(6)$ \\
H11 & 0.8219 & 0.2338 & 0.7126 & $0.016^{*}$ & $0.900(6)$ \\
C12 & $0.8306(11)$ & $0.2830(4)$ & $0.5549(3)$ & $0.0131(8)$ & $0.900(6)$ \\
C112 & $0.9006(7)$ & $0.1222(2)$ & $0.5455(3)$ & $0.0224(4)$ & $0.900(6)$ \\
C13 & $0.8071(8)$ & $0.3838(4)$ & $0.4674(3)$ & $0.0134(10)$ & $0.900(6)$ \\
H13 & 0.8313 & 0.3677 & 0.4001 & $0.016^{*}$ & $0.900(6)$ \\
C13A & $0.7467(12)$ & $0.5117(5)$ & $0.4780(3)$ & $0.0138(10)$ & $0.900(6)$ \\
C14 & $0.7273(10)$ & $0.6182(5)$ & $0.3794(3)$ & $0.0128(11)$ & $0.900(6)$ \\
O14 & $0.750(2)$ & $0.5900(6)$ & $0.2952(3)$ & $0.0201(10)$ & $0.900(6)$ \\
C15 & $0.675(2)$ & $0.7488(5)$ & $0.3946(4)$ & $0.0154(9)$ & $0.900(6)$ \\
C16 & $0.623(2)$ & $0.7619(4)$ & $0.4956(4)$ & $0.0146(12)$ & $0.900(6)$ \\
H16 & 0.5786 & 0.8476 & 0.5036 & $0.018^{*}$ & $0.900(6)$ \\
N17 & $0.6312(15)$ & $0.6629(4)$ & $0.5838(3)$ & $0.0129(7)$ & $0.900(6)$ \\
C18 & $0.5458(9)$ & $0.6900(5)$ & $0.6874(4)$ & $0.0151(8)$ & $0.900(6)$ \\
H18A & 0.4500 & 0.6222 & 0.7318 & $0.018^{*}$ & $0.900(6)$ \\
H18B & 0.4708 & 0.7777 & 0.6761 & $0.018^{*}$ & $0.900(6)$ \\
C18A & $0.7098(10)$ & $0.6883(4)$ & $0.7436(5)$ & $0.0147(9)$ & $0.900(6)$ \\
C19 & $0.7676(9)$ & $0.8073(4)$ & $0.7489(6)$ & $0.0198(14)$ & $0.900(6)$ \\
H19 & 0.6997 & 0.8888 & 0.7191 & $0.024^{*}$ & $0.900(6)$ \\
C110 & $0.9232(11)$ & $0.8066(5)$ & $0.7972(7)$ & $0.0250(13)$ & $0.900(6)$ \\
H110 & 0.9668 & 0.8877 & 0.7979 & $0.030^{*}$ & $0.900(6)$ \\
C111 & $1.0156(8)$ & $0.6872(5)$ & $0.8449(5)$ & $0.0244(12)$ & $0.900(6)$ \\
H111 & 1.1171 & 0.6862 & 0.8819 & $0.029^{*}$ & $0.900(6)$ \\
C112 & $0.9603(7)$ & $0.5682(5)$ & $0.8387(4)$ & $0.0201(11)$ & $0.900(6)$ \\
H112 & 1.0274 & 0.4871 & 0.8697 & $0.024^{*}$ & $0.900(6)$ \\
C12A & $0.8079(6)$ & $0.5675(4)$ & $0.7876(3)$ & $0.0155(10)$ & $0.900(6)$ \\
C113 & $0.7482(7)$ & $0.4385(4)$ & $0.7797(3)$ & $0.0164(9)$ & $0.900(6)$
\end{tabular}




\begin{tabular}{|c|c|c|c|c|c|}
\hline H113 & 0.8579 & 0.3720 & 0.8023 & $0.020 *$ & $0.900(6)$ \\
\hline $\mathrm{C} 13 \mathrm{C}$ & $0.7491(7)$ & $0.4319(4)$ & 0.6683 & $0.0132(10)$ & $0.900(6)$ \\
\hline C131 & $0.5547(6)$ & $0.3870(4)$ & 0.8619 & $0.0206(10)$ & $0.900(6)$ \\
\hline H13A & 0.4468 & 0.4590 & 0.8571 & $0.025^{*}$ & $0.900(6)$ \\
\hline H13B & 0.5095 & 0.3134 & 0.8439 & $0.025^{*}$ & $0.900(6)$ \\
\hline C132 & $0.5838(8)$ & $0.3386(6)$ & $0.9751(3)$ & $0.0223(14)$ & $0.900(6)$ \\
\hline $\mathrm{H} 13 \mathrm{C}$ & 0.6297 & 0.4106 & 0.9935 & $0.033^{*}$ & $0.900(6)$ \\
\hline H13D & 0.6849 & 0.2638 & 0.9816 & $0.033^{*}$ & $0.900(6)$ \\
\hline H13E & 0.4556 & 0.3101 & 1.0234 & $0.033^{*}$ & $0.900(6)$ \\
\hline C13B & $0.7097(12)$ & $0.5356(5)$ & $0.5784(3)$ & $0.0137(11)$ & $0.900(6)$ \\
\hline C151 & $0.662(2)$ & $0.8739(5)$ & $0.3103(4)$ & $0.0181(9)$ & $0.900(6)$ \\
\hline O151 & $0.6227(17)$ & $0.9825(6)$ & $0.3249(5)$ & $0.0256(15)$ & $0.900(6)$ \\
\hline O152 & $0.707(4)$ & $0.8574(8)$ & $0.2120(4)$ & $0.0237(10)$ & $0.900(6)$ \\
\hline C152 & $0.6960(10)$ & $0.9782(7)$ & $0.1264(4)$ & $0.0234(16)$ & $0.900(6)$ \\
\hline $\mathrm{H} 15 \mathrm{~A}$ & 0.7887 & 1.0424 & 0.1262 & $0.028 *$ & $0.900(6)$ \\
\hline H15B & 0.5571 & 1.0197 & 0.1342 & $0.028^{*}$ & $0.900(6)$ \\
\hline C153 & $0.757(3)$ & $0.9364(13)$ & $0.0249(5)$ & $0.029(2)$ & $0.900(6)$ \\
\hline $\mathrm{H} 15 \mathrm{C}$ & 0.7525 & 1.0143 & -0.0359 & $0.043 *$ & $0.900(6)$ \\
\hline H15D & 0.8948 & 0.8953 & 0.0186 & $0.043^{*}$ & $0.900(6)$ \\
\hline $\mathrm{H} 15 \mathrm{E}$ & 0.6645 & 0.8727 & 0.0265 & $0.043 *$ & $0.900(6)$ \\
\hline $\mathrm{C} 21$ & $0.822(8)$ & $0.330(3)$ & $0.645(2)$ & $0.0131(8)$ & $0.100(6)$ \\
\hline $\mathrm{H} 21$ & 0.8866 & 0.2706 & 0.6970 & $0.016^{*}$ & $0.100(6)$ \\
\hline $\mathrm{C} 22$ & $0.844(11)$ & $0.307(2)$ & $0.546(2)$ & $0.0131(8)$ & $0.100(6)$ \\
\hline $\mathrm{Cl} 22$ & $0.889(7)$ & $0.146(2)$ & $0.536(3)$ & $0.0224(4)$ & $0.100(6)$ \\
\hline $\mathrm{C} 23$ & $0.830(9)$ & $0.410(3)$ & $0.459(2)$ & $0.0134(10)$ & $0.100(6)$ \\
\hline $\mathrm{H} 23$ & 0.8925 & 0.4039 & 0.3896 & $0.016^{*}$ & $0.100(6)$ \\
\hline $\mathrm{C} 23 \mathrm{~A}$ & $0.720(12)$ & $0.527(3)$ & $0.474(2)$ & $0.0138(10)$ & $0.100(6)$ \\
\hline $\mathrm{C} 24$ & $0.694(12)$ & $0.634(4)$ & $0.377(2)$ & $0.0128(11)$ & $0.100(6)$ \\
\hline $\mathrm{O} 24$ & $0.73(2)$ & $0.607(5)$ & $0.291(3)$ & $0.0201(10)$ & $0.100(6)$ \\
\hline $\mathrm{C} 25$ & $0.68(2)$ & $0.766(3)$ & $0.390(3)$ & $0.0154(9)$ & $0.100(6)$ \\
\hline $\mathrm{C} 26$ & $0.64(2)$ & $0.780(3)$ & $0.490(3)$ & $0.0146(12)$ & $0.100(6)$ \\
\hline $\mathrm{H} 26$ & 0.6103 & 0.8675 & 0.4974 & $0.018 *$ & $0.100(6)$ \\
\hline $\mathrm{N} 27$ & $0.630(14)$ & 0.680 & $0.579(2)$ & $0.0129(7)$ & $0.100(6)$ \\
\hline $\mathrm{C} 28$ & $0.536(7)$ & $0.710(4)$ & $0.681(3)$ & $0.0151(8)$ & $0.100(6)$ \\
\hline $\mathrm{H} 28 \mathrm{~A}$ & 0.4242 & 0.6517 & 0.7199 & $0.018^{*}$ & $0.100(6)$ \\
\hline $\mathrm{H} 28 \mathrm{~B}$ & 0.4789 & 0.8031 & 0.6667 & $0.018^{*}$ & $0.100(6)$ \\
\hline C28A & $0.689(9)$ & $0.691(2)$ & $0.748(5)$ & $0.0147(9)$ & $0.100(6)$ \\
\hline $\mathrm{C} 29$ & $0.800(10)$ & $0.797(3)$ & $0.739(6)$ & $0.0198(14)$ & $0.100(6)$ \\
\hline H29 & 0.7762 & 0.8816 & 0.6922 & $0.024^{*}$ & $0.100(6)$ \\
\hline $\mathrm{C} 210$ & $0.945(11)$ & $0.778(4)$ & $0.798(7)$ & $0.0250(13)$ & $0.100(6)$ \\
\hline $\mathrm{H} 210$ & 0.9976 & 0.8513 & 0.8064 & $0.030^{*}$ & $0.100(6)$ \\
\hline C211 & $1.013(8)$ & $0.650(5)$ & $0.844(6)$ & $0.0244(12)$ & $0.100(6)$ \\
\hline H211 & 1.1323 & 0.6345 & 0.8710 & $0.029^{*}$ & $0.100(6)$ \\
\hline $\mathrm{C} 212$ & $0.908(7)$ & $0.543(4)$ & $0.850(4)$ & $0.0201(11)$ & $0.100(6)$ \\
\hline $\mathrm{H} 212$ & 0.9427 & 0.4563 & 0.8910 & $0.024 *$ & $0.100(6)$ \\
\hline $\mathrm{C} 22 \mathrm{~A}$ & $0.752(6)$ & $0.561(2)$ & 0.798 & $0.0155(10)$ & $0.100(6)$ \\
\hline C213 & $0.664(5)$ & $0.446(2)$ & $0.7812(17)$ & $0.0164(9)$ & $0.100(6)$ \\
\hline $\mathrm{H} 223$ & 0.5160 & 0.4694 & 0.7970 & $0.020 *$ & $0.100(6)$ \\
\hline
\end{tabular}




$\begin{array}{llllll}\mathrm{C} 23 \mathrm{C} & 0.704(8) & 0.441(3) & 0.6666(17) & 0.0132(10) & 0.100(6) \\ \mathrm{C} 231 & 0.684(5) & 0.314(2) & 0.8666(19) & 0.0206(10) & 0.100(6) \\ \mathrm{H} 23 \mathrm{~A} & 0.5920 & 0.2518 & 0.8633 & 0.025^{*} & 0.100(6) \\ \mathrm{H} 23 \mathrm{~B} & 0.8238 & 0.2748 & 0.8512 & 0.025^{*} & 0.100(6) \\ \mathrm{C} 232 & 0.636(11) & 0.330(5) & 0.9781(19) & 0.0223(14) & 0.100(6) \\ \mathrm{H} 23 \mathrm{C} & 0.6340 & 0.2425 & 1.0298 & 0.033^{*} & 0.100(6) \\ \mathrm{H} 23 \mathrm{D} & 0.5043 & 0.3775 & 0.9910 & 0.033^{*} & 0.100(6) \\ \mathrm{H} 23 \mathrm{E} & 0.7398 & 0.3803 & 0.9851 & 0.033^{*} & 0.100(6) \\ \mathrm{C} 23 \mathrm{~B} & 0.679(13) & 0.549(3) & 0.576(2) & 0.0137(11) & 0.100(6) \\ \mathrm{C} 251 & 0.66(2) & 0.891(4) & 0.305(3) & 0.0181(9) & 0.100(6) \\ \mathrm{O} 251 & 0.592(18) & 0.996(5) & 0.320(5) & 0.0256(15) & 0.100(6) \\ \mathrm{O} 252 & 0.70(4) & 0.871(7) & 0.208(4) & 0.0237(10) & 0.100(6) \\ \mathrm{C} 252 & 0.649(14) & 0.985(7) & 0.124(4) & 0.0234(16) & 0.100(6) \\ \mathrm{H} 25 \mathrm{~A} & 0.7156 & 1.0637 & 0.1225 & 0.028^{*} & 0.100(6) \\ \mathrm{H} 25 \mathrm{~B} & 0.5016 & 1.0068 & 0.1354 & 0.028^{*} & 0.100(6) \\ \mathrm{C} 253 & 0.73(3) & 0.947(12) & 0.022(4) & 0.029(2) & 0.100(6) \\ \mathrm{H} 25 \mathrm{C} & 0.7002 & 1.0204 & -0.0378 & 0.043^{*} & 0.100(6) \\ \mathrm{H} 25 \mathrm{D} & 0.8737 & 0.9256 & 0.0125 & 0.043^{*} & 0.100(6) \\ \mathrm{H} 25 \mathrm{E} & 0.6609 & 0.8689 & 0.0254 & 0.043^{*} & 0.100(6)\end{array}$

Atomic displacement parameters $\left(\AA^{2}\right)$

\begin{tabular}{|c|c|c|c|c|c|c|}
\hline & $U^{11}$ & $U^{22}$ & $U^{33}$ & $U^{12}$ & $U^{13}$ & $U^{23}$ \\
\hline C11 & $0.0130(16)$ & $0.0069(15)$ & 0.0188 (14) & $-0.0051(16)$ & $-0.0023(12)$ & $-0.0017(11)$ \\
\hline $\mathrm{C} 12$ & $0.0130(16)$ & $0.0069(15)$ & 0.0188 (14) & $-0.0051(16)$ & $-0.0023(12)$ & $-0.0017(11)$ \\
\hline $\mathrm{Cl} 12$ & $0.0303(8)$ & $0.0107(10)$ & $0.0255(9)$ & $0.0016(10)$ & $-0.0043(6)$ & $-0.0069(9)$ \\
\hline C13 & $0.008(2)$ & $0.017(2)$ & 0.0168 (19) & $-0.0060(19)$ & $0.0014(15)$ & $-0.0073(16)$ \\
\hline C13A & 0.010 & $0.016(2)$ & 0.0157 (18) & $-0.0007(16)$ & $-0.0053(14)$ & $-0.0035(15)$ \\
\hline C14 & $0.008(4)$ & $0.018(2)$ & $0.0136(17)$ & $0.0013(16)$ & $-0.0052(14)$ & $-0.0046(15)$ \\
\hline $\mathrm{O} 14$ & $0.024(3)$ & $0.021(2)$ & $0.0164(13)$ & $0.003(3)$ & $-0.0057(12)$ & $-0.0078(13)$ \\
\hline C15 & 0.0154 (19) & $0.017(2)$ & $0.0115(17)$ & $0.000(3)$ & $-0.0043(16)$ & $-0.0001(15)$ \\
\hline C16 & $0.015(3)$ & $0.012(2)$ & $0.0172(18)$ & $-0.001(3)$ & $-0.0053(17)$ & $-0.0032(16)$ \\
\hline N17 & $0.0182(16)$ & $0.0090(18)$ & $0.0106(14)$ & 0.0009 (19) & $-0.0040(13)$ & $-0.0014(12)$ \\
\hline C18 & 0.0159 (19) & $0.017(2)$ & $0.0121(18)$ & $0.0032(17)$ & $-0.0020(14)$ & $-0.0060(17)$ \\
\hline C18A & $0.014(2)$ & $0.0192(18)$ & $0.0096(17)$ & $0.0003(14)$ & $0.0009(17)$ & $-0.0049(14)$ \\
\hline C19 & $0.026(3)$ & 0.0171 (19) & $0.015(3)$ & $-0.0041(18)$ & $0.001(2)$ & $-0.0049(17)$ \\
\hline C110 & $0.028(3)$ & $0.026(3)$ & $0.024(2)$ & -0.010 & $-0.006(2)$ & $-0.009(3)$ \\
\hline C111 & $0.020(2)$ & $0.036(3)$ & $0.025(2)$ & $-0.007(2)$ & $-0.0062(17)$ & $-0.018(3)$ \\
\hline $\mathrm{C} 112$ & $0.013(3)$ & $0.027(3)$ & $0.018(2)$ & 0.0031 (19) & $-0.002(2)$ & -0.0055 (19) \\
\hline $\mathrm{C} 12 \mathrm{~A}$ & 0.015 & $0.0191(18)$ & $0.0097(18)$ & $-0.0027(16)$ & $0.0046(19)$ & $-0.0055(15)$ \\
\hline C113 & $0.020(2)$ & 0.0177 (19) & $0.0114(18)$ & $0.0014(17)$ & $-0.0055(17)$ & $-0.0029(14)$ \\
\hline $\mathrm{C} 13 \mathrm{C}$ & $0.012(3)$ & $0.0152(18)$ & $0.0138(17)$ & $-0.0004(15)$ & $-0.0044(15)$ & $-0.0042(14)$ \\
\hline C131 & $0.026(2)$ & $0.018(2)$ & $0.017(2)$ & $-0.0053(17)$ & $-0.0030(17)$ & $-0.0037(17)$ \\
\hline C132 & $0.027(4)$ & $0.022(2)$ & $0.0172(19)$ & $0.000(2)$ & $-0.0055(18)$ & $-0.0029(16)$ \\
\hline C13B & 0.008 (4) & 0.0157 (19) & $0.0180(17)$ & $-0.0012(15)$ & $-0.0036(15)$ & $-0.0046(15)$ \\
\hline C151 & $0.0148(18)$ & $0.021(2)$ & 0.0184 (19) & $-0.004(3)$ & $-0.0043(17)$ & $-0.0037(17)$ \\
\hline O151 & $0.040(5)$ & $0.0151(18)$ & $0.0198(15)$ & $0.0079(17)$ & $-0.0102(16)$ & $-0.0028(14)$ \\
\hline O152 & $0.039(4)$ & $0.018(2)$ & $0.0124(13)$ & $-0.001(3)$ & $-0.0069(15)$ & $-0.0001(13)$ \\
\hline
\end{tabular}




\begin{tabular}{|c|c|c|c|c|c|c|}
\hline C152 & $0.030(5)$ & $0.021(2)$ & $0.0154(18)$ & $0.000(3)$ & -0.008 (2) & $0.0024(16)$ \\
\hline C153 & $0.039(7)$ & $0.030(3)$ & 0.0157 (19) & $-0.016(3)$ & $-0.0037(18)$ & $0.0014(18)$ \\
\hline $\mathrm{C} 21$ & $0.0130(16)$ & $0.0069(15)$ & $0.0188(14)$ & $-0.0051(16)$ & $-0.0023(12)$ & $-0.0017(11)$ \\
\hline $\mathrm{C} 22$ & $0.0130(16)$ & $0.0069(15)$ & $0.0188(14)$ & $-0.0051(16)$ & $-0.0023(12)$ & $-0.0017(11)$ \\
\hline $\mathrm{Cl} 22$ & $0.0303(8)$ & $0.0107(10)$ & $0.0255(9)$ & $0.0016(10)$ & $-0.0043(6)$ & $-0.0069(9)$ \\
\hline $\mathrm{C} 23$ & $0.008(2)$ & $0.017(2)$ & $0.0168(19)$ & $-0.0060(19)$ & $0.0014(15)$ & $-0.0073(16)$ \\
\hline $\mathrm{C} 23 \mathrm{~A}$ & $0.010(3)$ & $0.016(2)$ & $0.0157(18)$ & $-0.0007(16)$ & $-0.0053(14)$ & $-0.0035(15)$ \\
\hline $\mathrm{C} 24$ & $0.008(4)$ & $0.018(2)$ & $0.0136(17)$ & $0.0013(16)$ & $-0.0052(14)$ & $-0.0046(15)$ \\
\hline $\mathrm{O} 24$ & $0.024(3)$ & $0.021(2)$ & $0.0164(13)$ & $0.003(3)$ & $-0.0057(12)$ & $-0.0078(13)$ \\
\hline $\mathrm{C} 25$ & 0.0154 (19) & $0.017(2)$ & $0.0115(17)$ & $0.000(3)$ & $-0.0043(16)$ & $-0.0001(15)$ \\
\hline $\mathrm{C} 26$ & $0.015(3)$ & $0.012(2)$ & $0.0172(18)$ & $-0.001(3)$ & $-0.0053(17)$ & $-0.0032(16)$ \\
\hline N27 & $0.0182(16)$ & $0.0090(18)$ & $0.0106(14)$ & 0.0009 (19) & $-0.0040(13)$ & $-0.0014(12)$ \\
\hline $\mathrm{C} 28$ & 0.0159 (19) & $0.017(2)$ & $0.0121(18)$ & $0.0032(17)$ & $-0.0020(14)$ & $-0.0060(17)$ \\
\hline $\mathrm{C} 28 \mathrm{~A}$ & $0.014(2)$ & $0.0192(18)$ & $0.0096(17)$ & $0.0003(14)$ & $0.0009(17)$ & $-0.0049(14)$ \\
\hline C29 & $0.026(3)$ & $0.0171(19)$ & $0.015(3)$ & $-0.0041(18)$ & $0.001(2)$ & $-0.0049(17)$ \\
\hline $\mathrm{C} 210$ & $0.028(3)$ & $0.026(3)$ & $0.024(2)$ & $-0.010(3)$ & $-0.006(2)$ & $-0.009(3)$ \\
\hline $\mathrm{C} 211$ & $0.020(2)$ & $0.036(3)$ & $0.025(2)$ & $-0.007(2)$ & $-0.0062(17)$ & $-0.018(3)$ \\
\hline $\mathrm{C} 212$ & $0.013(3)$ & $0.027(3)$ & $0.018(2)$ & $0.0031(19)$ & $-0.002(2)$ & $-0.0055(19)$ \\
\hline $\mathrm{C} 22 \mathrm{~A}$ & $0.015(3)$ & $0.0191(18)$ & $0.0097(18)$ & $-0.0027(16)$ & $0.0046(19)$ & $-0.0055(15)$ \\
\hline $\mathrm{C} 213$ & $0.020(2)$ & $0.0177(19)$ & $0.0114(18)$ & $0.0014(17)$ & $-0.0055(17)$ & $-0.0029(14)$ \\
\hline $\mathrm{C} 23 \mathrm{C}$ & $0.012(3)$ & $0.0152(18)$ & $0.0138(17)$ & $-0.0004(15)$ & $-0.0044(15)$ & $-0.0042(14)$ \\
\hline $\mathrm{C} 231$ & $0.026(2)$ & $0.018(2)$ & $0.017(2)$ & $-0.0053(17)$ & $-0.0030(17)$ & $-0.0037(17)$ \\
\hline $\mathrm{C} 232$ & $0.027(4)$ & $0.022(2)$ & $0.0172(19)$ & $0.000(2)$ & $-0.0055(18)$ & $-0.0029(16)$ \\
\hline C23B & $0.008(4)$ & $0.0157(19)$ & $0.0180(17)$ & $-0.0012(15)$ & $-0.0036(15)$ & $-0.0046(15)$ \\
\hline C251 & $0.0148(18)$ & $0.021(2)$ & $0.0184(19)$ & $-0.004(3)$ & $-0.0043(17)$ & $-0.0037(17)$ \\
\hline $\mathrm{O} 251$ & $0.040(5)$ & $0.0151(18)$ & $0.0198(15)$ & $0.0079(17)$ & $-0.0102(16)$ & $-0.0028(14)$ \\
\hline $\mathrm{O} 252$ & $0.039(4)$ & $0.018(2)$ & $0.0124(13)$ & $-0.001(3)$ & $-0.0069(15)$ & $-0.0001(13)$ \\
\hline $\mathrm{C} 252$ & $0.030(5)$ & $0.021(2)$ & $0.0154(18)$ & $0.000(3)$ & $-0.008(2)$ & $0.0024(16)$ \\
\hline C253 & $0.039(7)$ & $0.030(3)$ & $0.0157(19)$ & $-0.016(3)$ & $-0.0037(18)$ & $0.0014(18)$ \\
\hline
\end{tabular}

Geometric parameters $\left(\AA,{ }^{\circ}\right)$

\begin{tabular}{llll}
\hline $\mathrm{C} 11-\mathrm{C} 12$ & $1.393(5)$ & $\mathrm{C} 21-\mathrm{C} 23 \mathrm{C}$ & $1.401(11)$ \\
$\mathrm{C} 11-\mathrm{C} 13 \mathrm{C}$ & $1.399(5)$ & $\mathrm{C} 21-\mathrm{C} 22$ & $1.402(10)$ \\
$\mathrm{C} 11-\mathrm{H} 11$ & 0.9500 & $\mathrm{C} 21-\mathrm{H} 21$ & 0.9500 \\
$\mathrm{C} 12-\mathrm{C} 13$ & $1.364(5)$ & $\mathrm{C} 22-\mathrm{C} 23$ & $1.364(10)$ \\
$\mathrm{C} 12-\mathrm{C} 112$ & $1.746(4)$ & $\mathrm{C} 22-\mathrm{C} 22$ & $1.740(10)$ \\
$\mathrm{C} 13-\mathrm{C} 13 \mathrm{~A}$ & $1.405(5)$ & $\mathrm{C} 23-\mathrm{C} 23 \mathrm{~A}$ & $1.406(11)$ \\
$\mathrm{C} 13-\mathrm{H} 13$ & 0.9500 & $\mathrm{C} 23-\mathrm{H} 23$ & 0.9500 \\
$\mathrm{C} 13 \mathrm{~A}-\mathrm{C} 13 \mathrm{~B}$ & $1.416(5)$ & $\mathrm{C} 23 \mathrm{~A}-\mathrm{C} 23 \mathrm{~B}$ & $1.417(9)$ \\
$\mathrm{C} 13 \mathrm{~A}-\mathrm{C} 14$ & $1.489(5)$ & $\mathrm{C} 23 \mathrm{~A}-\mathrm{C} 24$ & $1.490(10)$ \\
$\mathrm{C} 14-\mathrm{O} 14$ & $1.236(4)$ & $\mathrm{C} 24-\mathrm{O} 24$ & $1.239(10)$ \\
$\mathrm{C} 14-\mathrm{C} 15$ & $1.448(5)$ & $\mathrm{C} 24-\mathrm{C} 25$ & $1.450(11)$ \\
$\mathrm{C} 15-\mathrm{C} 16$ & $1.374(5)$ & $\mathrm{C} 25-\mathrm{C} 26$ & $1.374(10)$ \\
$\mathrm{C} 15-\mathrm{C} 151$ & $1.477(5)$ & $\mathrm{C} 25-\mathrm{C} 251$ & $1.478(10)$ \\
$\mathrm{C} 16-\mathrm{N} 17$ & $1.340(5)$ & $\mathrm{C} 26-\mathrm{N} 27$ & $1.341(9)$ \\
$\mathrm{C} 16-\mathrm{H} 16$ & 0.9500 & $\mathrm{C} 26-\mathrm{H} 26$ & 0.9500 \\
$\mathrm{~N} 17-\mathrm{C} 13 \mathrm{~B}$ & $1.403(4)$ & $\mathrm{N} 27-\mathrm{C} 23 \mathrm{~B}$ & $1.403(10)$
\end{tabular}




\begin{tabular}{|c|c|c|c|}
\hline $\mathrm{N} 17-\mathrm{C} 18$ & $1.482(4)$ & N27-C28 & $1.484(10)$ \\
\hline $\mathrm{C} 18-\mathrm{C} 18 \mathrm{~A}$ & $1.500(5)$ & $\mathrm{C} 28-\mathrm{C} 28 \mathrm{~A}$ & $1.502(10)$ \\
\hline C18-H18A & 0.9900 & $\mathrm{C} 28-\mathrm{H} 28 \mathrm{~A}$ & 0.9900 \\
\hline C18-H18B & 0.9900 & $\mathrm{C} 28-\mathrm{H} 28 \mathrm{~B}$ & 0.9900 \\
\hline $\mathrm{C} 18 \mathrm{~A}-\mathrm{C} 19$ & $1.399(5)$ & $\mathrm{C} 28 \mathrm{~A}-\mathrm{C} 29$ & $1.400(11)$ \\
\hline $\mathrm{C} 18 \mathrm{~A}-\mathrm{C} 12 \mathrm{~A}$ & $1.400(5)$ & $\mathrm{C} 28 \mathrm{~A}-\mathrm{C} 22 \mathrm{~A}$ & $1.405(9)$ \\
\hline $\mathrm{C} 19-\mathrm{C} 110$ & $1.380(5)$ & $\mathrm{C} 29-\mathrm{C} 210$ & $1.381(10)$ \\
\hline C19-H19 & 0.9500 & $\mathrm{C} 29-\mathrm{H} 29$ & 0.9500 \\
\hline $\mathrm{C} 110-\mathrm{C} 111$ & $1.386(6)$ & $\mathrm{C} 210-\mathrm{C} 211$ & $1.387(11)$ \\
\hline $\mathrm{C} 110-\mathrm{H} 110$ & 0.9500 & $\mathrm{C} 210-\mathrm{H} 210$ & 0.9500 \\
\hline $\mathrm{C} 111-\mathrm{C} 112$ & $1.396(6)$ & $\mathrm{C} 211-\mathrm{C} 212$ & $1.396(11)$ \\
\hline C111-H111 & 0.9500 & $\mathrm{C} 211-\mathrm{H} 211$ & 0.9500 \\
\hline $\mathrm{C} 112-\mathrm{C} 12 \mathrm{~A}$ & $1.389(5)$ & $\mathrm{C} 212-\mathrm{C} 22 \mathrm{~A}$ & $1.387(10)$ \\
\hline C112-H112 & 0.9500 & $\mathrm{C} 212-\mathrm{H} 212$ & 0.9500 \\
\hline $\mathrm{C} 12 \mathrm{~A}-\mathrm{C} 113$ & $1.519(5)$ & $\mathrm{C} 22 \mathrm{~A}-\mathrm{C} 213$ & $1.521(10)$ \\
\hline $\mathrm{C} 113-\mathrm{C} 13 \mathrm{C}$ & $1.531(5)$ & $\mathrm{C} 213-\mathrm{C} 23 \mathrm{C}$ & $1.527(9)$ \\
\hline $\mathrm{C} 113-\mathrm{C} 131$ & $1.550(5)$ & $\mathrm{C} 213-\mathrm{C} 231$ & $1.540(10)$ \\
\hline C113-H113 & 1.0000 & $\mathrm{C} 213-\mathrm{H} 223$ & 1.0000 \\
\hline $\mathrm{C} 13 \mathrm{C}-\mathrm{C} 13 \mathrm{~B}$ & $1.430(5)$ & $\mathrm{C} 23 \mathrm{C}-\mathrm{C} 23 \mathrm{~B}$ & $1.438(9)$ \\
\hline $\mathrm{C} 131-\mathrm{C} 132$ & $1.519(5)$ & $\mathrm{C} 231-\mathrm{C} 232$ & $1.523(11)$ \\
\hline C131-H13A & 0.9900 & $\mathrm{C} 231-\mathrm{H} 23 \mathrm{~A}$ & 0.9900 \\
\hline C131-H13B & 0.9900 & $\mathrm{C} 231-\mathrm{H} 23 \mathrm{~B}$ & 0.9900 \\
\hline $\mathrm{C} 132-\mathrm{H} 13 \mathrm{C}$ & 0.9800 & $\mathrm{C} 232-\mathrm{H} 23 \mathrm{C}$ & 0.9800 \\
\hline C132-H13D & 0.9800 & $\mathrm{C} 232-\mathrm{H} 23 \mathrm{D}$ & 0.9800 \\
\hline $\mathrm{C} 132-\mathrm{H} 13 \mathrm{E}$ & 0.9800 & $\mathrm{C} 232-\mathrm{H} 23 \mathrm{E}$ & 0.9800 \\
\hline $\mathrm{C} 151-\mathrm{O} 151$ & $1.213(5)$ & $\mathrm{C} 251-\mathrm{O} 251$ & $1.214(11)$ \\
\hline $\mathrm{C} 151-\mathrm{O} 152$ & $1.352(4)$ & $\mathrm{C} 251-\mathrm{O} 252$ & $1.354(11)$ \\
\hline $\mathrm{O} 152-\mathrm{C} 152$ & $1.455(6)$ & $\mathrm{O} 252-\mathrm{C} 252$ & $1.455(12)$ \\
\hline $\mathrm{C} 152-\mathrm{C} 153$ & $1.517(5)$ & $\mathrm{C} 252-\mathrm{C} 253$ & $1.518(11)$ \\
\hline $\mathrm{C} 152-\mathrm{H} 15 \mathrm{~A}$ & 0.9900 & $\mathrm{C} 252-\mathrm{H} 25 \mathrm{~A}$ & 0.9900 \\
\hline $\mathrm{C} 152-\mathrm{H} 15 \mathrm{~B}$ & 0.9900 & $\mathrm{C} 252-\mathrm{H} 25 \mathrm{~B}$ & 0.9900 \\
\hline C153-H15C & 0.9800 & $\mathrm{C} 253-\mathrm{H} 25 \mathrm{C}$ & 0.9800 \\
\hline C153-H15D & 0.9800 & $\mathrm{C} 253-\mathrm{H} 25 \mathrm{D}$ & 0.9800 \\
\hline $\mathrm{C} 153-\mathrm{H} 15 \mathrm{E}$ & 0.9800 & $\mathrm{C} 253-\mathrm{H} 25 \mathrm{E}$ & 0.9800 \\
\hline $\mathrm{C} 12-\mathrm{C} 11-\mathrm{C} 13 \mathrm{C}$ & $122.4(3)$ & $\mathrm{H} 15 \mathrm{D}-\mathrm{C} 153-\mathrm{H} 15 \mathrm{E}$ & 109.5 \\
\hline $\mathrm{C} 12-\mathrm{C} 11-\mathrm{H} 11$ & 118.8 & $\mathrm{C} 23 \mathrm{C}-\mathrm{C} 21-\mathrm{C} 22$ & $119.8(13)$ \\
\hline $\mathrm{C} 13 \mathrm{C}-\mathrm{C} 11-\mathrm{H} 11$ & 118.8 & $\mathrm{C} 23 \mathrm{C}-\mathrm{C} 21-\mathrm{H} 21$ & 120.1 \\
\hline $\mathrm{C} 13-\mathrm{C} 12-\mathrm{C} 11$ & $121.0(3)$ & $\mathrm{C} 22-\mathrm{C} 21-\mathrm{H} 21$ & 120.1 \\
\hline $\mathrm{C} 13-\mathrm{C} 12-\mathrm{C} 112$ & $120.6(3)$ & $\mathrm{C} 23-\mathrm{C} 22-\mathrm{C} 21$ & $120.2(12)$ \\
\hline $\mathrm{C} 11-\mathrm{C} 12-\mathrm{Cl} 12$ & $118.4(3)$ & $\mathrm{C} 23-\mathrm{C} 22-\mathrm{Cl} 22$ & $120.5(12)$ \\
\hline $\mathrm{C} 12-\mathrm{C} 13-\mathrm{C} 13 \mathrm{~A}$ & $119.3(3)$ & $\mathrm{C} 21-\mathrm{C} 22-\mathrm{C} 122$ & $119.3(12)$ \\
\hline $\mathrm{C} 12-\mathrm{C} 13-\mathrm{H} 13$ & 120.4 & $\mathrm{C} 22-\mathrm{C} 23-\mathrm{C} 23 \mathrm{~A}$ & $118.0(14)$ \\
\hline $\mathrm{C} 13 \mathrm{~A}-\mathrm{C} 13-\mathrm{H} 13$ & 120.4 & $\mathrm{C} 22-\mathrm{C} 23-\mathrm{H} 23$ & 121.0 \\
\hline $\mathrm{C} 13-\mathrm{C} 13 \mathrm{~A}-\mathrm{C} 13 \mathrm{~B}$ & $120.4(3)$ & $\mathrm{C} 23 \mathrm{~A}-\mathrm{C} 23-\mathrm{H} 23$ & 121.0 \\
\hline $\mathrm{C} 13-\mathrm{C} 13 \mathrm{~A}-\mathrm{C} 14$ & $116.9(3)$ & $\mathrm{C} 23-\mathrm{C} 23 \mathrm{~A}-\mathrm{C} 23 \mathrm{~B}$ & $119.3(16)$ \\
\hline $\mathrm{C} 13 \mathrm{~B}-\mathrm{C} 13 \mathrm{~A}-\mathrm{C} 14$ & $122.7(3)$ & $\mathrm{C} 23-\mathrm{C} 23 \mathrm{~A}-\mathrm{C} 24$ & $116.5(14)$ \\
\hline $\mathrm{O} 14-\mathrm{C} 14-\mathrm{C} 15$ & $126.0(3)$ & $\mathrm{C} 23 \mathrm{~B}-\mathrm{C} 23 \mathrm{~A}-\mathrm{C} 24$ & $122.7(10)$ \\
\hline
\end{tabular}




\begin{tabular}{|c|c|}
\hline $\mathrm{O} 14-\mathrm{C} 14-\mathrm{C} 13 \mathrm{~A}$ & $119.8(3)$ \\
\hline $\mathrm{C} 15-\mathrm{C} 14-\mathrm{C} 13 \mathrm{~A}$ & $114.2(3)$ \\
\hline $\mathrm{C} 16-\mathrm{C} 15-\mathrm{C} 14$ & $119.3(3)$ \\
\hline $\mathrm{C} 16-\mathrm{C} 15-\mathrm{C} 151$ & $114.5(3)$ \\
\hline $\mathrm{C} 14-\mathrm{C} 15-\mathrm{C} 151$ & $126.0(3)$ \\
\hline $\mathrm{N} 17-\mathrm{C} 16-\mathrm{C} 15$ & $124.8(4)$ \\
\hline $\mathrm{N} 17-\mathrm{C} 16-\mathrm{H} 16$ & 117.6 \\
\hline $\mathrm{C} 15-\mathrm{C} 16-\mathrm{H} 16$ & 117.6 \\
\hline $\mathrm{C} 16-\mathrm{N} 17-\mathrm{C} 13 \mathrm{~B}$ & $121.1(3)$ \\
\hline $\mathrm{C} 16-\mathrm{N} 17-\mathrm{C} 18$ & $118.0(3)$ \\
\hline $\mathrm{C} 13 \mathrm{~B}-\mathrm{N} 17-\mathrm{C} 18$ & $120.8(3)$ \\
\hline $\mathrm{N} 17-\mathrm{C} 18-\mathrm{C} 18 \mathrm{~A}$ & $110.7(3)$ \\
\hline $\mathrm{N} 17-\mathrm{C} 18-\mathrm{H} 18 \mathrm{~A}$ & 109.5 \\
\hline $\mathrm{C} 18 \mathrm{~A}-\mathrm{C} 18-\mathrm{H} 18 \mathrm{~A}$ & 109.5 \\
\hline $\mathrm{N} 17-\mathrm{C} 18-\mathrm{H} 18 \mathrm{~B}$ & 109.5 \\
\hline $\mathrm{C} 18 \mathrm{~A}-\mathrm{C} 18-\mathrm{H} 18 \mathrm{~B}$ & 109.5 \\
\hline $\mathrm{H} 18 \mathrm{~A}-\mathrm{C} 18-\mathrm{H} 18 \mathrm{~B}$ & 108.1 \\
\hline $\mathrm{C} 19-\mathrm{C} 18 \mathrm{~A}-\mathrm{C} 12 \mathrm{~A}$ & $120.6(3)$ \\
\hline $\mathrm{C} 19-\mathrm{C} 18 \mathrm{~A}-\mathrm{C} 18$ & $119.9(3)$ \\
\hline $\mathrm{C} 12 \mathrm{~A}-\mathrm{C} 18 \mathrm{~A}-\mathrm{C} 18$ & $119.5(3)$ \\
\hline $\mathrm{C} 110-\mathrm{C} 19-\mathrm{C} 18 \mathrm{~A}$ & $120.1(4)$ \\
\hline $\mathrm{C} 110-\mathrm{C} 19-\mathrm{H} 19$ & 120.0 \\
\hline $\mathrm{C} 18 \mathrm{~A}-\mathrm{C} 19-\mathrm{H} 19$ & 120.0 \\
\hline $\mathrm{C} 19-\mathrm{C} 110-\mathrm{C} 111$ & $119.8(4)$ \\
\hline $\mathrm{C} 19-\mathrm{C} 110-\mathrm{H} 110$ & 120.1 \\
\hline $\mathrm{C} 111-\mathrm{C} 110-\mathrm{H} 110$ & 120.1 \\
\hline $\mathrm{C} 110-\mathrm{C} 111-\mathrm{C} 112$ & $120.3(4$ \\
\hline $\mathrm{C} 110-\mathrm{C} 111-\mathrm{H} 111$ & 119.8 \\
\hline $\mathrm{C} 112-\mathrm{C} 111-\mathrm{H} 111$ & 119.8 \\
\hline $\mathrm{C} 12 \mathrm{~A}-\mathrm{C} 112-\mathrm{C} 111$ & $120.6(4)$ \\
\hline $\mathrm{C} 12 \mathrm{~A}-\mathrm{C} 112-\mathrm{H} 112$ & 119.7 \\
\hline $\mathrm{C} 111-\mathrm{C} 112-\mathrm{H} 112$ & 119.7 \\
\hline $\mathrm{C} 112-\mathrm{C} 12 \mathrm{~A}-\mathrm{C} 18 \mathrm{~A}$ & $118.6(3)$ \\
\hline $\mathrm{C} 112-\mathrm{C} 12 \mathrm{~A}-\mathrm{C} 113$ & $120.8(3)$ \\
\hline $\mathrm{C} 18 \mathrm{~A}-\mathrm{C} 12 \mathrm{~A}-\mathrm{C} 113$ & $120.6(3)$ \\
\hline $\mathrm{C} 12 \mathrm{~A}-\mathrm{C} 113-\mathrm{C} 13 \mathrm{C}$ & $116.4(3)$ \\
\hline $\mathrm{C} 12 \mathrm{~A}-\mathrm{C} 113-\mathrm{C} 131$ & $113.0(3)$ \\
\hline $\mathrm{C} 13 \mathrm{C}-\mathrm{C} 113-\mathrm{C} 131$ & $112.9(3)$ \\
\hline $\mathrm{C} 12 \mathrm{~A}-\mathrm{C} 113-\mathrm{H} 113$ & 104.3 \\
\hline $\mathrm{C} 13 \mathrm{C}-\mathrm{C} 113-\mathrm{H} 113$ & 104.3 \\
\hline $\mathrm{C} 131-\mathrm{C} 113-\mathrm{H} 113$ & 104.3 \\
\hline $\mathrm{C} 11-\mathrm{C} 13 \mathrm{C}-\mathrm{C} 13 \mathrm{~B}$ & $116.6(3)$ \\
\hline $\mathrm{C} 11-\mathrm{C} 13 \mathrm{C}-\mathrm{C} 113$ & $114.0(3)$ \\
\hline $\mathrm{C} 13 \mathrm{~B}-\mathrm{C} 13 \mathrm{C}-\mathrm{C} 113$ & $129.4(3)$ \\
\hline $\mathrm{C} 132-\mathrm{C} 131-\mathrm{C} 113$ & $113.4(3)$ \\
\hline $\mathrm{C} 132-\mathrm{C} 131-\mathrm{H} 13 \mathrm{~A}$ & 108.9 \\
\hline $\mathrm{C} 113-\mathrm{C} 131-\mathrm{H} 13 \mathrm{~A}$ & 108.9 \\
\hline $\mathrm{C} 132-\mathrm{C} 131-\mathrm{H} 13 \mathrm{~B}$ & 108.9 \\
\hline
\end{tabular}

\begin{tabular}{|c|c|}
\hline $\mathrm{O} 24-\mathrm{C} 24-\mathrm{C} 25$ & $125(2)$ \\
\hline $\mathrm{O} 24-\mathrm{C} 24-\mathrm{C} 23 \mathrm{~A}$ & $118.9(17)$ \\
\hline $\mathrm{C} 25-\mathrm{C} 24-\mathrm{C} 23 \mathrm{~A}$ & $113.5(13)$ \\
\hline $\mathrm{C} 26-\mathrm{C} 25-\mathrm{C} 24$ & $118.8(16)$ \\
\hline $\mathrm{C} 26-\mathrm{C} 25-\mathrm{C} 251$ & $114.7(14)$ \\
\hline $\mathrm{C} 24-\mathrm{C} 25-\mathrm{C} 251$ & $125.3(18)$ \\
\hline $\mathrm{N} 27-\mathrm{C} 26-\mathrm{C} 25$ & $124.9(13)$ \\
\hline $\mathrm{N} 27-\mathrm{C} 26-\mathrm{H} 26$ & 117.5 \\
\hline $\mathrm{C} 25-\mathrm{C} 26-\mathrm{H} 26$ & 117.5 \\
\hline $\mathrm{C} 26-\mathrm{N} 27-\mathrm{C} 23 \mathrm{~B}$ & $121.3(11)$ \\
\hline $\mathrm{C} 26-\mathrm{N} 27-\mathrm{C} 28$ & $117.1(14)$ \\
\hline $\mathrm{C} 23 \mathrm{~B}-\mathrm{N} 27-\mathrm{C} 28$ & $120.8(16)$ \\
\hline $\mathrm{N} 27-\mathrm{C} 28-\mathrm{C} 28 \mathrm{~A}$ & $110.5(15)$ \\
\hline $\mathrm{N} 27-\mathrm{C} 28-\mathrm{H} 28 \mathrm{~A}$ & 109.5 \\
\hline $\mathrm{C} 28 \mathrm{~A}-\mathrm{C} 28-\mathrm{H} 28 \mathrm{~A}$ & 109.5 \\
\hline $\mathrm{N} 27-\mathrm{C} 28-\mathrm{H} 28 \mathrm{~B}$ & 109.5 \\
\hline $\mathrm{C} 28 \mathrm{~A}-\mathrm{C} 28-\mathrm{H} 28 \mathrm{~B}$ & 109.5 \\
\hline $\mathrm{H} 28 \mathrm{~A}-\mathrm{C} 28-\mathrm{H} 28 \mathrm{~B}$ & 108.1 \\
\hline $\mathrm{C} 29-\mathrm{C} 28 \mathrm{~A}-\mathrm{C} 22 \mathrm{~A}$ & $120.2(14)$ \\
\hline $\mathrm{C} 29-\mathrm{C} 28 \mathrm{~A}-\mathrm{C} 28$ & $119.8(15)$ \\
\hline $\mathrm{C} 22 \mathrm{~A}-\mathrm{C} 28 \mathrm{~A}-\mathrm{C} 28$ & $118.2(12)$ \\
\hline $\mathrm{C} 210-\mathrm{C} 29-\mathrm{C} 28 \mathrm{~A}$ & $119.7(15)$ \\
\hline $\mathrm{C} 210-\mathrm{C} 29-\mathrm{H} 29$ & 120.2 \\
\hline $\mathrm{C} 28 \mathrm{~A}-\mathrm{C} 29-\mathrm{H} 29$ & 120.2 \\
\hline $\mathrm{C} 29-\mathrm{C} 210-\mathrm{C} 211$ & $119.0(15)$ \\
\hline $\mathrm{C} 29-\mathrm{C} 210-\mathrm{H} 210$ & 120.5 \\
\hline $\mathrm{C} 211-\mathrm{C} 210-\mathrm{H} 210$ & 120.5 \\
\hline $\mathrm{C} 210-\mathrm{C} 211-\mathrm{C} 212$ & $120.0(14)$ \\
\hline $\mathrm{C} 210-\mathrm{C} 211-\mathrm{H} 211$ & 120.0 \\
\hline $\mathrm{C} 212-\mathrm{C} 211-\mathrm{H} 211$ & 120.0 \\
\hline $\mathrm{C} 22 \mathrm{~A}-\mathrm{C} 212-\mathrm{C} 211$ & $120.6(13)$ \\
\hline $\mathrm{C} 22 \mathrm{~A}-\mathrm{C} 212-\mathrm{H} 212$ & 119.7 \\
\hline $\mathrm{C} 211-\mathrm{C} 212-\mathrm{H} 212$ & 119.7 \\
\hline $\mathrm{C} 212-\mathrm{C} 22 \mathrm{~A}-\mathrm{C} 28 \mathrm{~A}$ & $118.3(11)$ \\
\hline $\mathrm{C} 212-\mathrm{C} 22 \mathrm{~A}-\mathrm{C} 213$ & $122.4(13)$ \\
\hline $\mathrm{C} 28 \mathrm{~A}-\mathrm{C} 22 \mathrm{~A}-\mathrm{C} 213$ & $118.9(11)$ \\
\hline $\mathrm{C} 22 \mathrm{~A}-\mathrm{C} 213-\mathrm{C} 23 \mathrm{C}$ & $115.5(14)$ \\
\hline $\mathrm{C} 22 \mathrm{~A}-\mathrm{C} 213-\mathrm{C} 231$ & 113.8 \\
\hline $\mathrm{C} 23 \mathrm{C}-\mathrm{C} 213-\mathrm{C} 231$ & $117.1(12)$ \\
\hline $\mathrm{C} 22 \mathrm{~A}-\mathrm{C} 213-\mathrm{H} 223$ & 102.5 \\
\hline $\mathrm{C} 23 \mathrm{C}-\mathrm{C} 213-\mathrm{H} 223$ & 102.5 \\
\hline $\mathrm{C} 231-\mathrm{C} 213-\mathrm{H} 223$ & 102.5 \\
\hline $\mathrm{C} 21-\mathrm{C} 23 \mathrm{C}-\mathrm{C} 23 \mathrm{~B}$ & $115.6(14)$ \\
\hline $\mathrm{C} 21-\mathrm{C} 23 \mathrm{C}-\mathrm{C} 213$ & $115.6(12)$ \\
\hline $\mathrm{C} 23 \mathrm{~B}-\mathrm{C} 23 \mathrm{C}-\mathrm{C} 213$ & $126.8(11)$ \\
\hline $\mathrm{C} 232-\mathrm{C} 231-\mathrm{C} 213$ & $112.8(15)$ \\
\hline $\mathrm{C} 232-\mathrm{C} 231-\mathrm{H} 23 \mathrm{~A}$ & 109.0 \\
\hline $\mathrm{C} 213-\mathrm{C} 231-\mathrm{H} 23 \mathrm{~A}$ & 109.0 \\
\hline
\end{tabular}




$\begin{array}{ll}\text { C113-C131-H13B } & 108.9 \\ \text { H13A-C131-H13B } & 107.7 \\ \text { C131-C132-H13C } & 109.5 \\ \text { C131-C132-H13D } & 109.5 \\ \text { H13C-C132-H13D } & 109.5 \\ \text { C131-C132-H13E } & 109.5 \\ \text { H13C-C132-H13E } & 109.5 \\ \text { H13D-C132-H13E } & 109.5 \\ \text { N17-C13B-C13A } & 116.7(3) \\ \text { N17-C13B-C13C } & 123.2(3) \\ \text { C13A-C13B-C13C } & 120.1(3) \\ \text { O151-C151-O152 } & 121.9(4) \\ \text { O151-C151-C15 } & 124.7(4) \\ \text { O152-C151-C15 } & 113.3(3) \\ \text { C151-O152-C152 } & 115.2(4) \\ \text { O152-C152-C153 } & 105.8(4) \\ \text { O152-C152-H15A } & 110.6 \\ \text { C153-C152-H15A } & 110.6 \\ \text { O152-C152-H15B } & 110.6 \\ \text { C153-C152-H15B } & 110.6 \\ \text { H15A-C152-H15B } & 108.7 \\ \text { C152-C153-H15C } & 109.5 \\ \text { C152-C153-H15D } & 109.5 \\ \text { H15C-C153-H15D } & 109.5 \\ \text { C152-C153-H15E } & 109.5 \\ \text { H15C-C153-H15E } & 109.5 \\ \text { C13C-C11-C12-C13 } & 0.6(7) \\ \text { C13C-C11-C12-C112 } & 179.3(5) \\ \text { C11-C12-C13-C13A } & -2.5(8) \\ \text { C112-C12-C13-C13A } & 178.7(6) \\ \text { C12-C13-C13A-C13B } & -0.3(9) \\ \text { C12-C13-C13A-C14 } & 178.9(6) \\ \text { C13-C13A-C14-O14 } & 5.3(11) \\ \text { C13B-C13A-C14-O14 } & -175.6(10) \\ \text { C13-C13A-C14-C15 } & -176.8(9) \\ \text { C13B-C13A-C14-C15 } & 2.3(9) \\ \text { O14-C14-C15-C16 } & 169.4(12) \\ \text { C13A-C14-C15-C16 } & -8.4(13) \\ \text { O14-C14-C15-C151 } & -7.4(16) \\ \text { C13A-C14-C15-C151 } & 174.8(10) \\ \text { C14-C15-C16-N17 } & 5.4(18) \\ \text { C151-C15-C16-N17 } & -177.5(11) \\ \text { C15-C16-N17-C13B } & 4.8(16) \\ \text { C15-C16-N17-C18 } & -172.5(11) \\ \text { C16-N17-C18-C18A } & -110.9(9) \\ \text { C13B-N17-C18-C18A } & 71.9(8) \\ \text { N17-C18-C18A-C19 } & 105.6(7)\end{array}$

$\begin{array}{ll}\mathrm{C} 232-\mathrm{C} 231-\mathrm{H} 23 \mathrm{~B} & 109.0 \\ \mathrm{C} 213-\mathrm{C} 231-\mathrm{H} 23 \mathrm{~B} & 109.0 \\ \mathrm{H} 23 \mathrm{~A}-\mathrm{C} 231-\mathrm{H} 23 \mathrm{~B} & 107.8 \\ \mathrm{C} 231-\mathrm{C} 232-\mathrm{H} 23 \mathrm{C} & 109.5 \\ \mathrm{C} 231-\mathrm{C} 232-\mathrm{H} 23 \mathrm{D} & 109.5 \\ \mathrm{H} 23 \mathrm{C}-\mathrm{C} 232-\mathrm{H} 23 \mathrm{D} & 109.5 \\ \mathrm{C} 231-\mathrm{C} 232-\mathrm{H} 23 \mathrm{E} & 109.5 \\ \mathrm{H} 23 \mathrm{C}-\mathrm{C} 232-\mathrm{H} 23 \mathrm{E} & 109.5 \\ \mathrm{H} 23 \mathrm{D}-\mathrm{C} 232-\mathrm{H} 23 \mathrm{E} & 109.5 \\ \mathrm{~N} 27-\mathrm{C} 23 \mathrm{~B}-\mathrm{C} 23 \mathrm{~A} & 116.5(11) \\ \mathrm{N} 27-\mathrm{C} 23 \mathrm{~B}-\mathrm{C} 23 \mathrm{C} & 123.4(13) \\ \mathrm{C} 23 \mathrm{~A}-\mathrm{C} 23 \mathrm{~B}-\mathrm{C} 23 \mathrm{C} & 119.9(10) \\ \mathrm{O} 251-\mathrm{C} 251-\mathrm{O} 252 & 121(2) \\ \mathrm{O} 251-\mathrm{C} 251-\mathrm{C} 25 & 125(2) \\ \mathrm{O} 252-\mathrm{C} 251-\mathrm{C} 25 & 112.5(15) \\ \mathrm{C} 251-\mathrm{O} 252-\mathrm{C} 252 & 115.3(17) \\ \mathrm{O} 252-\mathrm{C} 252-\mathrm{C} 253 & 105.7(14) \\ \mathrm{O} 252-\mathrm{C} 252-\mathrm{H} 25 \mathrm{~A} & 110.6 \\ \mathrm{C} 253-\mathrm{C} 252-\mathrm{H} 25 \mathrm{~A} & 110.6 \\ \mathrm{H} 25 \mathrm{~A}-\mathrm{C} 252-\mathrm{H} 25 \mathrm{~B} & 108.7 \\ \mathrm{C} 252-\mathrm{C} 253-\mathrm{H} 25 \mathrm{C} & 109.5 \\ \mathrm{C} 252-\mathrm{C} 253-\mathrm{H} 25 \mathrm{D} & 109.5 \\ \mathrm{H} 25 \mathrm{C}-\mathrm{C} 253-\mathrm{H} 25 \mathrm{D} & 109.5 \\ \mathrm{C} 252-\mathrm{C} 253-\mathrm{H} 25 \mathrm{E} & 109.5 \\ \mathrm{H} 25 \mathrm{D}-\mathrm{C} 253-\mathrm{H} 25 \mathrm{E} & 109.5 \\ & \end{array}$

$\mathrm{C} 15-\mathrm{C} 151-\mathrm{O} 152-\mathrm{C} 152 \quad 179.9$ (16)

$\mathrm{C} 151-\mathrm{O} 152-\mathrm{C} 152-\mathrm{C} 153 \quad 178.5$ (16)

$\mathrm{C} 23 \mathrm{C}-\mathrm{C} 21-\mathrm{C} 22-\mathrm{C} 23 \quad 28(6)$

$\mathrm{C} 23 \mathrm{C}-\mathrm{C} 21-\mathrm{C} 22-\mathrm{C} 22 \quad-152(6)$

$\mathrm{C} 21-\mathrm{C} 22-\mathrm{C} 23-\mathrm{C} 23 \mathrm{~A} \quad-24(7)$

$\mathrm{C} 122-\mathrm{C} 22-\mathrm{C} 23-\mathrm{C} 23 \mathrm{~A} \quad 155(6)$

$\mathrm{C} 22-\mathrm{C} 23-\mathrm{C} 23 \mathrm{~A}-\mathrm{C} 23 \mathrm{~B} \quad 18(8)$

$\mathrm{C} 22-\mathrm{C} 23-\mathrm{C} 23 \mathrm{~A}-\mathrm{C} 24 \quad-175$ (6)

$\mathrm{C} 23-\mathrm{C} 23 \mathrm{~A}-\mathrm{C} 24-\mathrm{O} 24 \quad 14$ (11)

$\mathrm{C} 23 \mathrm{~B}-\mathrm{C} 23 \mathrm{~A}-\mathrm{C} 24-\mathrm{O} 24 \quad 180$ (9)

$\mathrm{C} 23-\mathrm{C} 23 \mathrm{~A}-\mathrm{C} 24-\mathrm{C} 25 \quad-150(8)$

$\mathrm{C} 23 \mathrm{~B}-\mathrm{C} 23 \mathrm{~A}-\mathrm{C} 24-\mathrm{C} 25 \quad 16(8)$

$\mathrm{O} 24-\mathrm{C} 24-\mathrm{C} 25-\mathrm{C} 26 \quad-179(12)$

$\mathrm{C} 23 \mathrm{~A}-\mathrm{C} 24-\mathrm{C} 25-\mathrm{C} 26 \quad-17(12)$

$\mathrm{O} 24-\mathrm{C} 24-\mathrm{C} 25-\mathrm{C} 251 \quad 14(15)$

$\mathrm{C} 23 \mathrm{~A}-\mathrm{C} 24-\mathrm{C} 25-\mathrm{C} 251 \quad 176(9)$

$\mathrm{C} 251-\mathrm{C} 25-\mathrm{C} 26-\mathrm{N} 27 \quad 176(11)$

$\mathrm{C} 25-\mathrm{C} 26-\mathrm{N} 27-\mathrm{C} 23 \mathrm{~B} \quad 4$ (16)

$\mathrm{C} 25-\mathrm{C} 26-\mathrm{N} 27-\mathrm{C} 28 \quad-166(10)$

$\mathrm{C} 26-\mathrm{N} 27-\mathrm{C} 28-\mathrm{C} 28 \mathrm{~A} \quad-116(8)$

$\mathrm{C} 23 \mathrm{~B}-\mathrm{N} 27-\mathrm{C} 28-\mathrm{C} 28 \mathrm{~A} \quad 74$ (6) 


\begin{tabular}{|c|c|}
\hline $\mathrm{N} 17-\mathrm{C} 18-\mathrm{C} 18 \mathrm{~A}-\mathrm{C} 12 \mathrm{~A}$ & $-72.5(6)$ \\
\hline $\mathrm{C} 12 \mathrm{~A}-\mathrm{C} 18 \mathrm{~A}-\mathrm{C} 19-\mathrm{C} 110$ & $0.4(10)$ \\
\hline $\mathrm{C} 18-\mathrm{C} 18 \mathrm{~A}-\mathrm{C} 19-\mathrm{C} 110$ & $-177.7(7)$ \\
\hline $\mathrm{C} 18 \mathrm{~A}-\mathrm{C} 19-\mathrm{C} 110-\mathrm{C} 111$ & $-3.0(11)$ \\
\hline $\mathrm{C} 19-\mathrm{C} 110-\mathrm{C} 111-\mathrm{C} 112$ & $3.8(11)$ \\
\hline $\mathrm{C} 110-\mathrm{C} 111-\mathrm{C} 112-\mathrm{C} 12 \mathrm{~A}$ & $-2.0(9)$ \\
\hline $\mathrm{C} 111-\mathrm{C} 112-\mathrm{C} 12 \mathrm{~A}-\mathrm{C} 18 \mathrm{~A}$ & $-0.7(7)$ \\
\hline $\mathrm{C} 111-\mathrm{C} 112-\mathrm{C} 12 \mathrm{~A}-\mathrm{C} 113$ & $179.4(5)$ \\
\hline $\mathrm{C} 19-\mathrm{C} 18 \mathrm{~A}-\mathrm{C} 12 \mathrm{~A}-\mathrm{C} 112$ & $1.5(8)$ \\
\hline $\mathrm{C} 18-\mathrm{C} 18 \mathrm{~A}-\mathrm{C} 12 \mathrm{~A}-\mathrm{C} 112$ & $179.6(5)$ \\
\hline $\mathrm{C} 19-\mathrm{C} 18 \mathrm{~A}-\mathrm{C} 12 \mathrm{~A}-\mathrm{C} 113$ & $-178.6(5)$ \\
\hline $\mathrm{C} 18-\mathrm{C} 18 \mathrm{~A}-\mathrm{C} 12 \mathrm{~A}-\mathrm{C} 113$ & $-0.5(8)$ \\
\hline $\mathrm{C} 112-\mathrm{C} 12 \mathrm{~A}-\mathrm{C} 113-\mathrm{C} 13 \mathrm{C}$ & $-126.3(4)$ \\
\hline $\mathrm{C} 18 \mathrm{~A}-\mathrm{C} 12 \mathrm{~A}-\mathrm{C} 113-\mathrm{C} 13 \mathrm{C}$ & $53.8(6)$ \\
\hline $\mathrm{C} 112-\mathrm{C} 12 \mathrm{~A}-\mathrm{C} 113-\mathrm{C} 131$ & $100.6(4)$ \\
\hline $\mathrm{C} 18 \mathrm{~A}-\mathrm{C} 12 \mathrm{~A}-\mathrm{C} 113-\mathrm{C} 131$ & $-79.3(6)$ \\
\hline $\mathrm{C} 12-\mathrm{C} 11-\mathrm{C} 13 \mathrm{C}-\mathrm{C} 13 \mathrm{~B}$ & $4.0(7)$ \\
\hline $\mathrm{C} 12-\mathrm{C} 11-\mathrm{C} 13 \mathrm{C}-\mathrm{C} 113$ & $-173.9(4)$ \\
\hline $\mathrm{C} 12 \mathrm{~A}-\mathrm{C} 113-\mathrm{C} 13 \mathrm{C}-\mathrm{C} 11$ & $146.8(4)$ \\
\hline $\mathrm{C} 131-\mathrm{C} 113-\mathrm{C} 13 \mathrm{C}-\mathrm{C} 11$ & $-80.1(5)$ \\
\hline $\mathrm{C} 12 \mathrm{~A}-\mathrm{C} 113-\mathrm{C} 13 \mathrm{C}-\mathrm{C} 13 \mathrm{~B}$ & $-30.8(7)$ \\
\hline $\mathrm{C} 131-\mathrm{C} 113-\mathrm{C} 13 \mathrm{C}-\mathrm{C} 13 \mathrm{~B}$ & $102.3(6)$ \\
\hline $\mathrm{C} 12 \mathrm{~A}-\mathrm{C} 113-\mathrm{C} 131-\mathrm{C} 132$ & $-69.4(5)$ \\
\hline $\mathrm{C} 13 \mathrm{C}-\mathrm{C} 113-\mathrm{C} 131-\mathrm{C} 132$ & $155.8(4)$ \\
\hline $\mathrm{C} 16-\mathrm{N} 17-\mathrm{C} 13 \mathrm{~B}-\mathrm{C} 13 \mathrm{~A}$ & $-10.6(12)$ \\
\hline $\mathrm{C} 18-\mathrm{N} 17-\mathrm{C} 13 \mathrm{~B}-\mathrm{C} 13 \mathrm{~A}$ & $166.5(7)$ \\
\hline $\mathrm{C} 16-\mathrm{N} 17-\mathrm{C} 13 \mathrm{~B}-\mathrm{C} 13 \mathrm{C}$ & $170.4(10)$ \\
\hline $\mathrm{C} 18-\mathrm{N} 17-\mathrm{C} 13 \mathrm{~B}-\mathrm{C} 13 \mathrm{C}$ & $-12.4(11)$ \\
\hline $\mathrm{C} 13-\mathrm{C} 13 \mathrm{~A}-\mathrm{C} 13 \mathrm{~B}-\mathrm{N} 17$ & $-174.0(8)$ \\
\hline $\mathrm{C} 14-\mathrm{C} 13 \mathrm{~A}-\mathrm{C} 13 \mathrm{~B}-\mathrm{N} 17$ & $6.9(10)$ \\
\hline $\mathrm{C} 13-\mathrm{C} 13 \mathrm{~A}-\mathrm{C} 13 \mathrm{~B}-\mathrm{C} 13 \mathrm{C}$ & $5.0(10)$ \\
\hline $\mathrm{C} 14-\mathrm{C} 13 \mathrm{~A}-\mathrm{C} 13 \mathrm{~B}-\mathrm{C} 13 \mathrm{C}$ & $-174.1(7)$ \\
\hline $\mathrm{C} 11-\mathrm{C} 13 \mathrm{C}-\mathrm{C} 13 \mathrm{~B}-\mathrm{N} 17$ & $172.2(7)$ \\
\hline $\mathrm{C} 113-\mathrm{C} 13 \mathrm{C}-\mathrm{C} 13 \mathrm{~B}-\mathrm{N} 17$ & $-10.2(10)$ \\
\hline $\mathrm{C} 11-\mathrm{C} 13 \mathrm{C}-\mathrm{C} 13 \mathrm{~B}-\mathrm{C} 13 \mathrm{~A}$ & $-6.7(9)$ \\
\hline $\mathrm{C} 113-\mathrm{C} 13 \mathrm{C}-\mathrm{C} 13 \mathrm{~B}-\mathrm{C} 13 \mathrm{~A}$ & $170.9(6)$ \\
\hline $\mathrm{C} 16-\mathrm{C} 15-\mathrm{C} 151-\mathrm{O} 151$ & $4.8(16)$ \\
\hline $\mathrm{C} 14-\mathrm{C} 15-\mathrm{C} 151-\mathrm{O} 151$ & $-178.3(15)$ \\
\hline $\mathrm{C} 16-\mathrm{C} 15-\mathrm{C} 151-\mathrm{O} 152$ & $-177.8(16)$ \\
\hline $\mathrm{C} 14-\mathrm{C} 15-\mathrm{C} 151-\mathrm{O} 152$ & $-0.9(16)$ \\
\hline $\mathrm{O} 151-\mathrm{C} 151-\mathrm{O} 152-\mathrm{C} 152$ & $-3(2)$ \\
\hline
\end{tabular}

$\begin{array}{ll}\mathrm{N} 27-\mathrm{C} 28-\mathrm{C} 28 \mathrm{~A}-\mathrm{C} 29 & 90(7) \\ \mathrm{N} 27-\mathrm{C} 28-\mathrm{C} 28 \mathrm{~A}-\mathrm{C} 22 \mathrm{~A} & -74(4) \\ \mathrm{C} 22 \mathrm{~A}-\mathrm{C} 28 \mathrm{~A}-\mathrm{C} 29-\mathrm{C} 210 & -14(10) \\ \mathrm{C} 28-\mathrm{C} 28 \mathrm{~A}-\mathrm{C} 29-\mathrm{C} 210 & -178(7) \\ \mathrm{C} 28 \mathrm{~A}-\mathrm{C} 29-\mathrm{C} 210-\mathrm{C} 211 & 16(12) \\ \mathrm{C} 29-\mathrm{C} 210-\mathrm{C} 211-\mathrm{C} 212 & -14(12) \\ \mathrm{C} 210-\mathrm{C} 211-\mathrm{C} 212-\mathrm{C} 22 \mathrm{~A} & 9(10) \\ \mathrm{C} 211-\mathrm{C} 212-\mathrm{C} 22 \mathrm{~A}-\mathrm{C} 28 \mathrm{~A} & -7(8) \\ \mathrm{C} 211-\mathrm{C} 212-\mathrm{C} 22 \mathrm{~A}-\mathrm{C} 213 & 166(5) \\ \mathrm{C} 29-\mathrm{C} 28 \mathrm{~A}-\mathrm{C} 22 \mathrm{~A}-\mathrm{C} 212 & 9(8) \\ \mathrm{C} 28-\mathrm{C} 28 \mathrm{~A}-\mathrm{C} 22 \mathrm{~A}-\mathrm{C} 212 & 174(5) \\ \mathrm{C} 29-\mathrm{C} 28 \mathrm{~A}-\mathrm{C} 22 \mathrm{~A}-\mathrm{C} 213 & -164(5) \\ \mathrm{C} 28-\mathrm{C} 28 \mathrm{~A}-\mathrm{C} 22 \mathrm{~A}-\mathrm{C} 213 & 1(7) \\ \mathrm{C} 212-\mathrm{C} 22 \mathrm{~A}-\mathrm{C} 213-\mathrm{C} 23 \mathrm{C} & -112(5) \\ \mathrm{C} 28 \mathrm{~A}-\mathrm{C} 22 \mathrm{~A}-\mathrm{C} 213-\mathrm{C} 23 \mathrm{C} & 60(4) \\ \mathrm{C} 212-\mathrm{C} 22 \mathrm{~A}-\mathrm{C} 213-\mathrm{C} 231 & 27(5) \\ \mathrm{C} 28 \mathrm{~A}-\mathrm{C} 22 \mathrm{~A}-\mathrm{C} 213-\mathrm{C} 231 & -160(4) \\ \mathrm{C} 22-\mathrm{C} 21-\mathrm{C} 23 \mathrm{C}-\mathrm{C} 23 \mathrm{~B} & -23(6) \\ \mathrm{C} 22-\mathrm{C} 21-\mathrm{C} 23 \mathrm{C}-\mathrm{C} 213 & 171(3) \\ \mathrm{C} 22 \mathrm{~A}-\mathrm{C} 213-\mathrm{C} 23 \mathrm{C}-\mathrm{C} 21 & 116(4) \\ \mathrm{C} 231-\mathrm{C} 213-\mathrm{C} 23 \mathrm{C}-\mathrm{C} 21 & -22(5) \\ \mathrm{C} 22 \mathrm{~A}-\mathrm{C} 213-\mathrm{C} 23 \mathrm{C}-\mathrm{C} 23 \mathrm{~B} & -47(6) \\ \mathrm{C} 231-\mathrm{C} 213-\mathrm{C} 23 \mathrm{C}-\mathrm{C} 23 \mathrm{~B} & 175(5) \\ \mathrm{C} 22 \mathrm{~A}-\mathrm{C} 213-\mathrm{C} 231-\mathrm{C} 232 & 42(5) \\ \mathrm{C} 23 \mathrm{C}-\mathrm{C} 213-\mathrm{C} 231-\mathrm{C} 232 & -179(4) \\ \mathrm{C} 26-\mathrm{N} 27-\mathrm{C} 23 \mathrm{~B}-\mathrm{C} 23 \mathrm{~A} & -5(11) \\ \mathrm{C} 28-\mathrm{N} 27-\mathrm{C} 23 \mathrm{~B}-\mathrm{C} 23 \mathrm{~A} & 165(6) \\ \mathrm{C} 26-\mathrm{N} 27-\mathrm{C} 23 \mathrm{~B}-\mathrm{C} 23 \mathrm{C} & 170(10) \\ \mathrm{C} 28-\mathrm{N} 27-\mathrm{C} 23 \mathrm{~B}-\mathrm{C} 23 \mathrm{C} & -20(11) \\ \mathrm{C} 23-\mathrm{C} 23 \mathrm{~A}-\mathrm{C} 23 \mathrm{~B}-\mathrm{N} 27 & 160(7) \\ \mathrm{C} 24-\mathrm{C} 23 \mathrm{~A}-\mathrm{C} 23 \mathrm{~B}-\mathrm{N} 27 & -6(10) \\ \mathrm{C} 23-\mathrm{C} 23 \mathrm{~A}-\mathrm{C} 23 \mathrm{~B}-\mathrm{C} 23 \mathrm{C} & -15(9) \\ \mathrm{C} 24-\mathrm{C} 23 \mathrm{~A}-\mathrm{C} 23 \mathrm{~B}-\mathrm{C} 23 \mathrm{C} & 179(7) \\ \mathrm{C} 21-\mathrm{C} 23 \mathrm{C}-\mathrm{C} 23 \mathrm{~B}-\mathrm{N} 27 & -157(7) \\ \mathrm{C} 213-\mathrm{C} 23 \mathrm{C}-\mathrm{C} 23 \mathrm{~B}-\mathrm{N} 27 & 6(10) \\ \mathrm{C} 21-\mathrm{C} 23 \mathrm{C}-\mathrm{C} 23 \mathrm{~B}-\mathrm{C} 23 \mathrm{~A} & 18(8) \\ \mathrm{C} 213-\mathrm{C} 23 \mathrm{C}-\mathrm{C} 23 \mathrm{~B}-\mathrm{C} 23 \mathrm{~A} & -179(5) \\ \mathrm{C} 24-\mathrm{C} 25-\mathrm{C} 251-\mathrm{O} 251 & 157(14) \\ \mathrm{C} 25-\mathrm{C} 25-\mathrm{C} 251-\mathrm{O} 252-\mathrm{O} 252 & -177(15) \\ \mathrm{C} 251-\mathrm{O} 252-\mathrm{C} 252-\mathrm{C} 253 & 170(16) \\ & 171(16)\end{array}$

Hydrogen-bond geometry $\left(\AA,{ }^{\circ}\right)$

\begin{tabular}{lllll}
\hline$D-\mathrm{H} \cdots A$ & $D-\mathrm{H}$ & $\mathrm{H} \cdots A$ & $D \cdots A$ & $D-\mathrm{H} \cdots A$ \\
\hline $\mathrm{C} 18-\mathrm{H} 18 B \cdots \mathrm{O} 151^{\mathrm{i}}$ & 0.99 & 2.54 & $3.483(9)$ & 159 \\
$\mathrm{C} 19-\mathrm{H} 19 \cdots \mathrm{O} 151^{\mathrm{i}}$ & 0.95 & 2.54 & $3.396(12)$ & 149
\end{tabular}


supporting information

$\mathrm{C} 28-\mathrm{H} 28 B^{\cdots} \cdot \mathrm{O} 251^{\mathrm{i}}$

$\mathrm{C} 29-\mathrm{H} 29 \cdots \mathrm{O} 251^{\mathrm{i}}$

Symmetry code: (i) $-x+1,-y+2,-z+1$.
0.99

0.95
2.10

2.75

$3.13(7)$

$3.38(13)$
159

124 